\title{
Radiological Conditions at the Southern Islands of Rongelap Atoll
}

William L. Robison

Cynthia L. Conrado

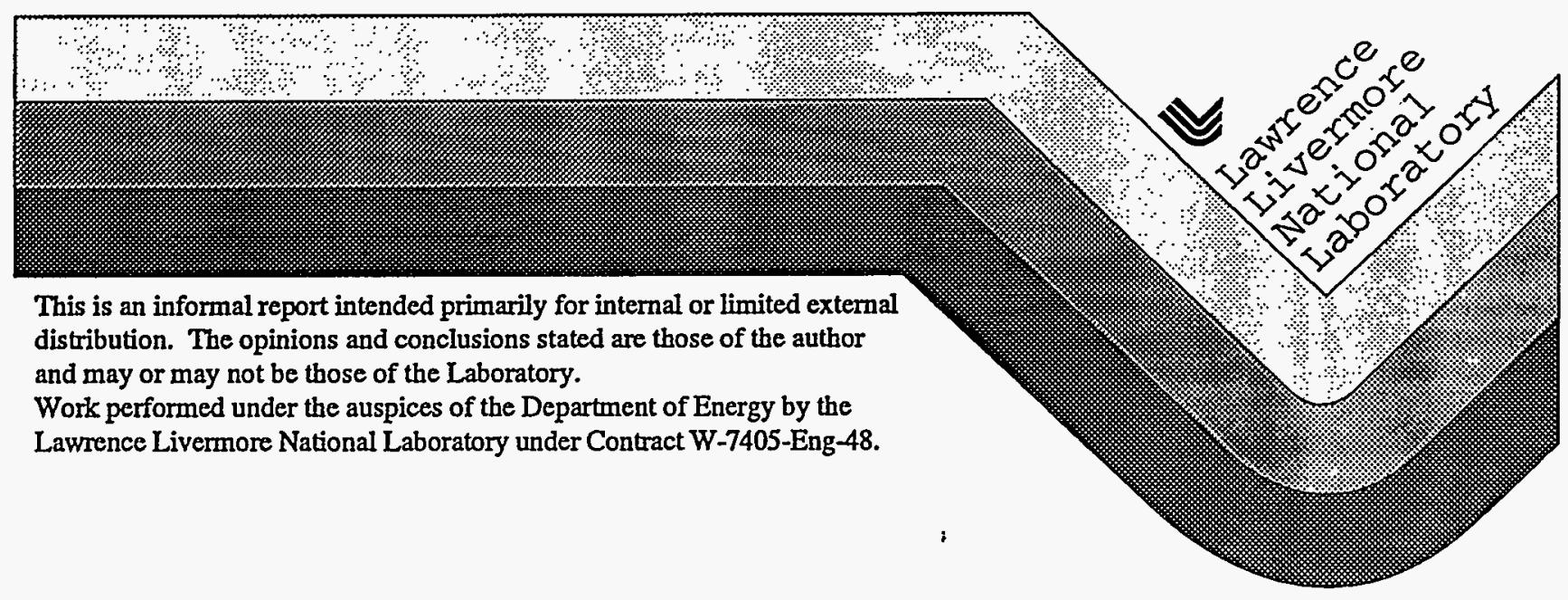


This document was prepared as an account of work sponsored by an agency of the United States Government. Neither the United States Government nor the University of Califomia nor any of their employees, makes any warranty, express or implied, or assumes any legal liability or responsibility for the accuracy, completeness, or usefulness of any information, apparatus, product, or process disclosed, or represents that its use would not infringe privately owned rights. Reference herein to any specific commercial product, process, or service by trade name, trademark, manufacturer, or otherwise, does not necessarily constitute or imply its endorsement, recommendation, or favoring by the United States Government or the University of California. The views and opinions of authors expressed herein do not necessarily state or reflect those of the United States Government or the University of California, and shall not be used for advertising or product endorsement purposes.

This report has been reproduced directly from the best available copy.

Available to DOE and DOE contractors from the Office of Scientific and Technical Information P.O. Box 62, Oak Ridge, TN 37831

Prices available from (615) 576-8401, FTS 626-8401

Available to the public from the

National Technical Information Service

U.S. Department of Commerce

5285 Port Royal Rd.

Springfield, VA 22161 


\section{DISCLAIMER}

Portions of this document may be illegible in electronic image products. Images are produced from the best available original document. 
Radiological Conditions at the Southern Islands of Rongelap Atoll

William L. Robison

Cynthia L. Conrado

Lawrence Livermore National Laboratory

Environmental Sciences Division

Report to DOE Environment, Safety, and Health

for Dr. Harry Pettengill, March 1992

March, 1996 


\section{TABLE OF CONTENTS}

Introductory Comments .01

Table 1 The number of food crops collected at the southern islands at Rongelap Atoll and the mean and median ${ }^{137} \mathrm{Cs}$ concentrations.

Table 2 The number of indicator species collected at the southern islands at Rongelap Atoll and the mean and median ${ }^{137} \mathrm{Cs}$ concentrations.

Table 3 The number of animal samples collected at the southern islands at Rongelap Atoll and the mean and median ${ }^{137} \mathrm{Cs}$ concentrations.

Table 4 The number of soil samples collected at the southern islands at Rongelap Atoll. 07

Table 5 Average ${ }^{137} \mathrm{Cs},{ }^{90} \mathrm{Sr},{ }^{239+240} \mathrm{Pu}$, and ${ }^{241} \mathrm{Am}$ soil concentrations in $\mathrm{pCi} / \mathrm{g}$ dry weight for soil profiles taken on Erapuotsu Island (F32). 08

Table 6

Average ${ }^{137} \mathrm{Cs}, 90 \mathrm{Sr}, 239+240 \mathrm{Pu}$, and ${ }^{241} \mathrm{Am}$ soil concentrations in $\mathrm{pCi} / \mathrm{g}$ dry weight for soil profiles taken on Eniaetok Island (F33).

Table 7

Average ${ }^{137} \mathrm{Cs},{ }^{90} \mathrm{Sr},{ }^{239+240} \mathrm{Pu}$, and ${ }^{241} \mathrm{Am}$ soil concentrations in $\mathrm{pCig/dry} \mathrm{weight} \mathrm{for} \mathrm{soil} \mathrm{profiles} \mathrm{taken} \mathrm{on} \mathrm{Busch} \mathrm{Island} \mathrm{(F38).}$

Table $8 \quad$ Average ${ }^{137} \mathrm{Cs},{ }^{90} \mathrm{Sr},{ }^{239}+240 \mathrm{Pu}$, and ${ }^{241} \mathrm{Am}$ soil concentrations in $\mathrm{pCi} / \mathrm{g}$ dry weight for soil profiles taken on Rongelap Island (F42).

Table 9 Average ${ }^{137} \mathrm{Cs},{ }^{90} \mathrm{Sr},{ }^{239+240} \mathrm{Pu}$, and ${ }^{241} \mathrm{Am}$ soil concentrations in $\mathrm{pCi} / \mathrm{g}$ dry weight for soil profiles taken on Arbar Island (F43).

Table 10 Average ${ }^{137} \mathrm{Cs},{ }^{90} \mathrm{Sr},{ }^{239}+240 \mathrm{Pu}$, and ${ }^{241}$. Am soil concentrations in $\mathrm{pCi} / \mathrm{g}$ dry weight for soil profiles taken on Eniran Island (F46). 
Table 11 Average ${ }^{137} \mathrm{Cs},{ }^{90} \mathrm{Sr},{ }^{239}+240 \mathrm{Pu}$, and ${ }^{241} \mathrm{Am}$ soil concentrations in $\mathrm{pCi} / \mathrm{g}$ dry weight for soil profiles taken on Tufa Island (F47).

Table 12 Average ${ }^{137} \mathrm{Cs},{ }^{90} \mathrm{Sr},{ }^{239}+240 \mathrm{Pu}$, and ${ }^{241} \mathrm{Am}$ soil concentrations in $\mathrm{pCi} / \mathrm{g}$ dry weight for soil profiles taken on Borukka Island (F49).

Figure 1 Rongelap Island- ${ }^{137} \mathrm{Cs}$ distribution in soil.............................. 12

Figure $2 \quad{ }^{137} \mathrm{Cs}$ in soil at southern islands of Rongelap Atoll................... 13

Appendix A Radionuclide concentration summary in vegetation samples collected during the 1978 NMIRS and from 1986 through 1991 in the southern islands of Rongelap Atoll. ..A-14

Table A-1 The ${ }^{137} \mathrm{Cs}$ radionuclide concentration summary for vegetation (food crops) collected from the southern islands of Rongelap Atoll during the 1978 NMIRS together with our recent trips in 1986 through 1991. A-15

Table A-2 The ${ }^{137} \mathrm{Cs}$ radionuclide concentration summary for vegetation (indicator species) collected from the southern islands of Rongelap Atoll during the 1978 NMIRS together with our recent trips in 1986 through 1991.

Appendix B Radionuclide concentration summary in animal samples collected during the 1978 NMIRS and from 1986 through 1991 in the southern islands of Rongelap Atoll. B-18

Table B-1 The ${ }^{137} \mathrm{Cs}$ radionuclide concentration summary for animals (food source) collected from the southern islands of Rongelap Atoll during the 1978 NMIRS together with our recent trips in 1986 through 1991.

Appendix C Radionuclide concentration summary of all soil profile samples collected during the 1978 NMIRS and from 1986 through 1991 in the southern islands of Rongelap Atoll.... C-20

Table C-1 Cesium-137 radionuclide concentration summary for all soil profiles taken from our most recent trip in 1991 on Erapuotsu Island (F32). C-21 
Table C-2 Cesium-137 radionuclide concentration summary for all soil profiles taken from the 1978 NMIRS together with our most recent trip in 1989 on Eniaetok Island (F33)

Table C-3 Cesium-137 radionuclide concentration summary for all soil profiles taken from our most recent trip in 1991 on Busch Island (F38).

Table C-4 Cesium-137 radionuclide concentration summary for all soil profiles taken from the 1978 NMIRS together with our most recent trips in 1986 through 1989 on Rongelap Island (F42).

Table C-5 Cesium-137 radionuclide concentration summary for all soil profiles taken from the 1978 NMIRS together with our most recent trip in 1991 on Arbar Island (F43).

Table C-6 Cesium-137 radionuclide concentration summary for all soil profiles taken from our most recent trip in 1991 on Eniran Island (F46).

Table C-7 Cesium-137 radionuclide concentration summary for all soil profiles taken from our most recent trip in 1991 on Tufa Island (F47). C-27

Table C-8 Cesium-137 radionuclide concentration summary for all soil profiles taken from the 1978 NMIRS together with our most recent trip in 1989 on Borukka Island (F49). C-28

Table C-9 Strontium-90 radionuclide concentration summary for all soil profiles taken during the 1978 NMIRS on Eniaetok Island (F33).

Table C-10 Strontium-90 radionuclide concentration summary for all soil profiles taken during the 1978 NMIRS on Rongelap Island (F42). C-30

Table C-11 Strontium-90 radionuclide concentration summary for all soil profiles taken during the 1978 NMIRS on Borukka Island (F49). C-31

Table C-12 Plutonium $239+240$ radionuclide concentration summary for all soil profiles taken during the 1978 NMIRS together with our recent trip in 1989 on Eniaetok Island (F33) C-32 
Table C-13 Plutonium $239+240$ radionuclide concentration summary for all soil profiles taken during the 1978 NMIRS together with our recent trip in 1986 on Rongelap Island (F42). C-33

Table C-14 Plutonium 239+240 radionuclide concentration summary for all soil profiles taken during the 1978 NMIRS together with our recent trip in 1989 on Borukka Island (F49). C-34

Table C-15 Americium-241 radionuclide concentration summary for all soil profiles taken from our recent trip in 1991 on Erapuotsu Island (F32). C-35

Table C-16 Americium-241 radionuclide concentration summary for all soil profiles taken from the 1978 NMIRS together with our most recent trip in 1989 on Eniaetok Island (F33). C-36

Table C-17 Americium-241 radionuclide concentration summary for all soil profiles taken from our most recent trip in 1991 on Busch Island (F38). C-37

Table C-18 Americium-241 radionuclide concentration summary for all soil profiles taken from the 1978 NMIRS together with our most recent trips in 1986 through 1989 on Rongelap Island (F42). C-38

Table C-19 Americium-241 radionuclide concentration summary for all soil profiles taken from the 1978 NMIRS together with our most recent trip in 1991 on Arbar Island (F43). C-39

Table C-20 Americium-241 radionuclide concentration summary for all soil profiles taken from our most recent trip in 1991 on Eniran Island (F46). $C-40$

Table C-21 Americium-241 radionuclide concentration summary for all soil profiles taken from our most recent trip in 1991 on Tufa Island (F47).....................................................................................

Table C-22 Americium-241 radionuclide concentration summary for all soil profiles taken from the 1978 NMIRS together with our most recent trip in 1989 on Borukka Island (F49)................... C-42

Appendix D Maps of Islands D-43 


\section{INTRODUCTORY COMMENTS}

The data presented in the following tables is the total available for each southern island; they include both the data from the 1978 Northern Marshall Island Radiological Survey (NMIRS) and trips to Rongelap Atoll from 1986 through 1991. There are additional samples that were taken at Rongelap Island in 1990 and 1991, and the data are unavailable for this report.

In one table we present the number of vegetation samples collected in the 1978 NMIRS and from 1986 through 1991. Again, the majority of the ${ }^{137} \mathrm{Cs}$ is from the 1986-1991 trips. We have not made additional analyses of $239+240 \mathrm{Pu}, 241 \mathrm{Am}$ and ${ }^{90} \mathrm{Sr}$ because the concentrations are very low and these radionuclides contribute less than $5 \%$ of an already very small dose.

In another table we show the number of soil samples collected at each island in 1978 and the number collected since 1986. Most of the data are from 1986 through 1991. The major exception is ${ }^{90} \mathrm{Sr}$ where all of the data are from the 1978 NMIRS. We have done some additional $\mathrm{Pu}$ analyses of soils from Rongelap Eniaetok, and Borukka Island but none of the other southern islands. A significant amount of new data for ${ }^{137} \mathrm{Cs}$ and ${ }^{241} \mathrm{Am}$ have been generated from the samples collected from 1986 through 1991.

The data are presented in the form of summary tables, graphics, detailed appendices and aerial photographs of the islands with the sample locations marked. The identified sample locations from the 1978 NMIRS will be added later. 
Table 1. The number of food crops collected at the southern islands at Rongelap Atoll and the mean and median ${ }^{137} \mathrm{Cs}$ concentrations.

\begin{tabular}{|c|c|c|c|c|c|c|}
\hline \multirow{2}{*}{$\begin{array}{l}\text { Island } \\
\text { Name }\end{array}$} & \multirow{2}{*}{$\begin{array}{c}\text { Island } \\
\text { Code }\end{array}$} & \multicolumn{3}{|c|}{ Number of Samples } & \multicolumn{2}{|c|}{ Cs-137 pCi/g wet wt. } \\
\hline & & Tota & NMIRS & $1986-1991$ & median & mean \\
\hline \multicolumn{7}{|c|}{ Drinking Coconut Meat } \\
\hline Erapuotsu & $32 f$ & 3 & 0 & 3 & 2.0 & 2.2 \\
\hline Eniaetok & $33 f$ & 7 & 2 & 5 & 1.7 & 2.7 \\
\hline Busch & $38 f$ & 5 & 0 & 5 & 1.1 & 1.1 \\
\hline Rongelap & $42 f$ & 200 & 3 & 197 & 1.4 & 1.9 \\
\hline Arbar & $43 f$ & 4 & 1 & 3 & 0.88 & 0.93 \\
\hline Eniran & $46 f$ & 4 & 0 & 4 & 0.98 & 1.1 \\
\hline Tufa & $47 f$ & 6 & 0 & 6 & 0.74 & 0.78 \\
\hline Borukka & $49 f$ & 5 & 0 & 5 & 1.2 & 1.3 \\
\hline \multicolumn{7}{|c|}{ Drinking Coconut Juice } \\
\hline Erapuotsu & $32 f$ & 3 & 0 & 3 & 0.79 & 0.85 \\
\hline Eniaetok & $33 f$ & 7 & 2 & 5 & 0.32 & 0.56 \\
\hline Busch & $38 \mathrm{f}$ & 5 & 0 & 5 & 0.28 & 0.32 \\
\hline Rongelap & $42 f$ & 194 & 3 & 191 & 0.62 & 0.85 \\
\hline Arbar & $43 f$ & 4 & 1 & 3 & 0.17 & 0.17 \\
\hline Eniran & $46 f$ & 4 & 0 & 4 & 0.47 & 0.48 \\
\hline Tufa & $47 f$ & 7 & 0 & 7 & 0.21 & 0.22 \\
\hline Borukka & $49 f$ & 5 & 0 & 5 & 0.36 & 0.46 \\
\hline \multicolumn{7}{|c|}{ Copra Meat } \\
\hline Eniaetok & $33 \mathrm{f}$ & 14 & 2 & 12 & 3.3 & 3.4 \\
\hline Rongelap & $42 f$ & 72 & 14 & 58 & 2.6 & 3.4 \\
\hline Arbar & $43 f$ & 1 & 0 & 1 & 0.82 & 0.82 \\
\hline Tufa & $47 f$ & 1 & 0 & 1 & 1.1 & 1.1 \\
\hline Borukka & $49 f$ & 6 & 1 & 5 & 2.0 & 2.9 \\
\hline \multicolumn{7}{|c|}{ Copra Juice } \\
\hline Eniaetok & $33 \mathrm{f}$ & 14 & 2 & 12 & 2.4 & 2.5 \\
\hline Rongelap & $42 f$ & 70 & 13 & 57 & 1.4 & 1.9 \\
\hline Arbar & $43 f$ & 1 & 0 & 1 & 1.1 & 1.1 \\
\hline Tufa & $47 f$ & 1 & 0 & 1 & 0.80 & 0.80 \\
\hline Borukka & $49 f$ & 6 & 1 & 5 & 1.2 & 2.1 \\
\hline
\end{tabular}


Table 1. The number of food crops collected at the southern islands at Rongelap Atoll and the mean and median ${ }^{137} \mathrm{Cs}$ concentrations (continued).

\begin{tabular}{|c|c|c|c|c|c|c|}
\hline \multirow{2}{*}{$\begin{array}{l}\text { Island } \\
\text { Name }\end{array}$} & \multirow{2}{*}{$\begin{array}{l}\text { Island } \\
\text { Code }\end{array}$} & \multicolumn{3}{|c|}{ Number of Samples } & \multicolumn{2}{|c|}{ Cs-137pCi / g wet wt. } \\
\hline & & Total & 1978 NMIRS & $1986-1991$ & median & mean \\
\hline \multicolumn{7}{|c|}{ Pandanus } \\
\hline Erapuotsu & $32 f$ & 3 & 0 & 3 & 2.0 & 2.4 \\
\hline Eniaetok & $33 f$ & 15 & 2 & 13 & 2.2 & 4.0 \\
\hline Busch & $38 \mathrm{f}$ & 2 & 0 & 2 & 0.83 & 0.83 \\
\hline Rongelap & $42 f$ & 78 & 16 & 62 & 5.9 & 7.6 \\
\hline Arbar & $43 \mathrm{f}$ & 4 & 2 & 2 & 1.4 & 3.1 \\
\hline Tufa & $47 \mathrm{f}$ & 2 & 0 & 2 & 4.0 & 4.0 \\
\hline Borukka & $49 f$ & 9 & 4 & 5 & 1.0 & 1.4 \\
\hline \multicolumn{7}{|c|}{ Breadfruit Meat } \\
\hline Rongelap & $42 f$ & 30 & 1 & 29 & 3.0 & 3.2 \\
\hline \multicolumn{7}{|c|}{ Tacca Meat } \\
\hline Busch & $38 \mathrm{f}$ & 1 & 0 & 1 & 2.3 & 2.3 \\
\hline Rongelap & $42 f$ & 5 & 0 & 5 & 4.3 & 5.7 \\
\hline Tufa & $47 f$ & 2 & 0 & 2 & 1.6 & 1.6 \\
\hline Borukka & $49 f$ & 4 & 1 & 3 & 1.2 & 1.2 \\
\hline \multicolumn{7}{|c|}{ Lime Meat } \\
\hline Rongelap & $42 f$ & 9 & 0 & 9 & 1.5 & 1.6 \\
\hline \multicolumn{7}{|c|}{ Squash Meat } \\
\hline Rongelap & $42 f$ & 2 & 0 & 2 & 5.8 & 5.8 \\
\hline
\end{tabular}


Table 2. The number of indicator species collected at the southern islands at Rongelap Atoll and the mean and median ${ }^{137} \mathrm{Cs}$ concentrations.

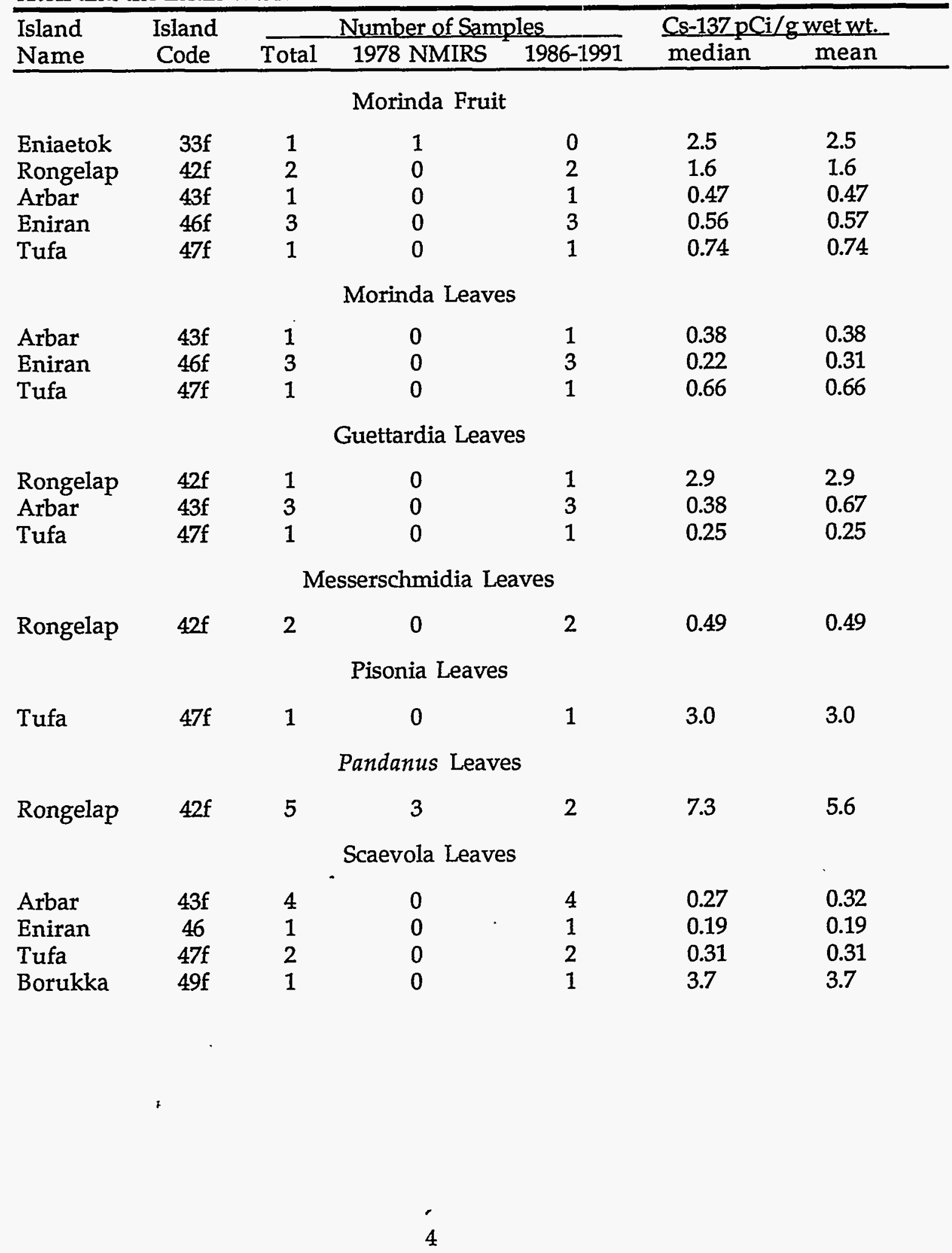


Table 2. The number of indicator species collected at the southern islands at Rongelap Atoll and the mean and median ${ }^{137} \mathrm{Cs}$ concentrations (continued).

\begin{tabular}{|c|c|c|c|c|c|c|}
\hline $\begin{array}{l}\text { Island } \\
\text { Name }\end{array}$ & $\begin{array}{l}\text { Island } \\
\text { Code }\end{array}$ & \multicolumn{3}{|c|}{ Number of Samples } & \multicolumn{2}{|c|}{$\mathrm{Cs}-137 \mathrm{pCi} / \mathrm{g}$ wet wt. } \\
\hline \multicolumn{7}{|c|}{ At-At Vine } \\
\hline Tufa & $47 f$ & 1 & 0 & 1 & 0.34 & 0.34 \\
\hline \multicolumn{7}{|c|}{ Tabero Fruit } \\
\hline Rongelap & $42 f$ & 3 & 0 & 3 & 2.0 & 1.8 \\
\hline \multicolumn{7}{|c|}{ Tabero Leaves } \\
\hline Rongelap & $42 f$ & 1 & 0 & 1 & 1.2 & 1.2 \\
\hline \multicolumn{7}{|c|}{ Coconut Fronds } \\
\hline Rongelap & $42 f$ & 16 & 0 & 16 & 1.1 & 2.4 \\
\hline
\end{tabular}


Table 3. The number of animal samples collected at the southern islands at Rongelap Atoll and the mean and median ${ }^{137} \mathrm{Cs}$ concentrations.

\begin{tabular}{|c|c|c|c|c|c|c|}
\hline \multirow{2}{*}{$\begin{array}{l}\text { Island } \\
\text { Name }\end{array}$} & \multirow{2}{*}{$\begin{array}{l}\text { Island } \\
\text { Code }\end{array}$} & \multicolumn{3}{|c|}{ Number of Samples } & \multicolumn{2}{|c|}{ Cs-137 pCi/g wet wt. } \\
\hline & & Total & 1978 NMIRS & $1986-1991$ & median & mean \\
\hline \multicolumn{7}{|c|}{ Coconut Crab Muscle } \\
\hline Erapuotsu & $32 f$ & 1 & 0 & 1 & 4.9 & 4.9 \\
\hline Busch & $38 f$ & 1 & 0 & 1 & 1.4 & 1.4 \\
\hline Arbar & $43 \mathrm{f}$ & 2 & 2 & 0 & 2.5 & 2.5 \\
\hline Eniran & $46 f$ & 2 & 0 & 2 & 1.5 & 1.5 \\
\hline Tufa & $47 f$ & 2 & 0 & 2 & 1.6 & 1.6 \\
\hline Borukka & $49 f$ & 1 & 0 & 1 & 2.1 & 2.1 \\
\hline
\end{tabular}

Coconut Crab Hepatopancreas

$\begin{array}{lllllll}\text { Erapuotsu } & 32 \mathrm{f} & 1 & 0 & 1 & 3.4 & 3.4 \\ \text { Busch } & 38 \mathrm{f} & 1 & 0 & 1 & 0.98 & 0.98 \\ \text { Eniran } & 46 \mathrm{f} & 2 & 0 & 2 & 1.1 & 1.1 \\ \text { Tufa } & 47 \mathrm{f} & 2 & 0 & 2 & 1.1 & 1.1 \\ \text { Borukka } & 49 \mathrm{f} & 1 & 0 & 1 & 1.9 & 1.9\end{array}$

Pig Heart

$\begin{array}{lllllll}\text { Rongelap } & 42 \mathrm{f} & 4 & 0 & 4 & 13 & 14\end{array}$

Pig Liver

$\begin{array}{lllllll}\text { Rongelap } & 42 f & 6 & 2 & 4 & 5.3 & 5.6\end{array}$

Pig Muscle

\begin{tabular}{lllllll} 
Rongelap & $42 \mathrm{f}$ & 7 & 2 & 5 & 13 & 14 \\
\hline
\end{tabular}

NOTE: Specific activity decay corrected to 1994. 
Table 4. The number of soil samples collected at the southern islands at Rongelap Atoll.

Number of Soil Samples for Cs-137 and Am-241

\begin{tabular}{|c|c|c|c|c|c|}
\hline \multirow{2}{*}{$\begin{array}{l}\text { Island } \\
\text { Name }\end{array}$} & \multirow{2}{*}{$\begin{array}{c}\text { Island } \\
\text { Code }\end{array}$} & \multicolumn{2}{|c|}{1978} & \multicolumn{2}{|c|}{ 1986-1991 } \\
\hline & & Profiles & $0-5 \mathrm{~cm}$. & Profiles & $0-5 \mathrm{Cl}$ \\
\hline Erapuotsu & $32 f$ & 0 & 0 & 3 & 3 \\
\hline Eniaetok & $33 f$ & 6 & 6 & 15 & 15 \\
\hline Busch & $38 \mathrm{f}$ & 0 & 0 & 5 & 5 \\
\hline Rongelap & $42 f$ & 28 & 27 & 90 & 267 \\
\hline Arbar & $43 \mathrm{f}$ & 7 & 6 & 7 & 7 \\
\hline Eniran & $46 f$ & 0 & 0 & 7 & 6 \\
\hline Tufa & $47 f$ & 0 & 0 & 8 & 8 \\
\hline Borukka & $49 f$ & 3 & 3 & 10 & 10 \\
\hline
\end{tabular}

Island - Island

Name Code

Number of Soil Samples for Pu-239+240

$\mathrm{cm}$.

\begin{tabular}{|c|c|c|c|c|c|}
\hline Erapuotsu & $32 f$ & 0 & 0 & 0 & 0 \\
\hline Eniaetok & $33 f$ & 6 & 6 & 0 & 15 \\
\hline Busch & $38 \mathrm{f}$ & 0 & 0 & 0 & 0 \\
\hline Rongelap & $42 f$ & 20 & 18 & 0 & 59 \\
\hline Arbar & $43 \mathrm{f}$ & 0 & 0 & 0 & 0 \\
\hline Eniran & $46 f$ & 0 & 0 & 0 & 0 \\
\hline Tufa & $47 f$ & 0 & 0 & 0 & 0 \\
\hline \multirow[t]{2}{*}{ Borukka } & $49 f$ & 3 & 3 & 0 & 10 \\
\hline & & \multicolumn{4}{|c|}{ Number of Soil Samples for Sr-90 } \\
\hline Island & Island & \multicolumn{2}{|c|}{1978} & \multicolumn{2}{|c|}{ 1986-1991 } \\
\hline $\begin{array}{l}\text { Name } \\
\mathrm{cm} \text {. }\end{array}$ & Code & Profiles & $0-5 \mathrm{~cm}$. & Profiles & $0-5$ \\
\hline Erapuotsu & $32 f$ & 0 & 0 & 0 & 0 \\
\hline Eniaetok & $33 f$ & 6 & 6 & 0 & 0 \\
\hline Busch & $38 \mathrm{f}$ & 0 & 0 & 0 & 0 \\
\hline Rongelap & $42 f$ & 21 & 20 & 0 & 0 \\
\hline Arbar & $43 f$ & 0 & 0 & 0 & 0 \\
\hline Eniran & $46 f$ & 0 & 0 & 0 & 0 \\
\hline Tufa & $47 f$ & 0 & 0 & 0 & 0 \\
\hline Borukka & $49 f$ & 3 & 3 & 0 & 0 \\
\hline
\end{tabular}


Table 5. Average ${ }^{137} \mathrm{Cs},{ }^{90} \mathrm{Sr},{ }^{239+240} \mathrm{Pu}$, and ${ }^{241} \mathrm{Am}$ soil concentrations in $\mathrm{pCi} / \mathrm{g}$ dry weight for soil profiles taken on Erapuotsu Island (F32).

\begin{tabular}{|c|c|c|c|c|c|c|c|c|}
\hline \multirow{2}{*}{$\begin{array}{l}\text { Soil } \\
\text { depth } \\
\text { (cm) }\end{array}$} & \multicolumn{2}{|c|}{${ }^{137} \mathrm{Cs}$} & \multicolumn{2}{|c|}{${ }^{90} \mathrm{Sr}$} & \multicolumn{2}{|c|}{$239+240 \mathrm{Pu}$} & \multicolumn{2}{|c|}{${ }^{241} \mathrm{Am}$} \\
\hline & Median & Mean & Median & Mean & Median & Mean & Median & Mean \\
\hline $00-05$ & 22 & 23 & NDA & NDA & NDA & NDA & 5.2 & 7.7 \\
\hline $05-10$ & 31 & 35 & NDA & NDA & NDA & NDA & 4.5 & 4.5 \\
\hline $10-15$ & 5 & 8.1 & NDA & NDA & NDA & NDA & 0.0 & 0.0 \\
\hline $15-25$ & 1.6 & 1.4 & NDA & NDA & NDA & NDA & 0.0 & 0.0 \\
\hline $25-40$ & 0.86 & 0.96 & NDA & NDA & NDA & NDA & 0.0 & 0.0 \\
\hline $40-60$ & 0.0 & 0.0 & NDA & NDA & NDA & NDA & 0.0 & 0.0 \\
\hline $00-05$ & 22 & 23 & NDA & NDA & NDA & NDA & 5.2 & 7.7 \\
\hline $00-10$ & 26 & 29 & NDA & NDA & NDA & NDA & 4.4 & 4.4 \\
\hline $00-15$ & 18 & 22 & NDA & NDA & NDA & NDA & 0.0 & 0.0 \\
\hline $00-25$ & 11 & 14 & $\mathrm{NDA}$ & NDA & $\mathrm{NDA}$ & NDA & 0.0 & 0.0 \\
\hline $00-40$ & 7.4 & 9.0 & NDA & NDA & NDA & NDA & 0.0 & 0.0 \\
\hline $00-60$ & 0.0 & 0.0 & NDA & NDA & NDA & NDA & 0.0 & 0.0 \\
\hline
\end{tabular}

NOTE: Specific activity decay corrected to 1994

$\infty$

NDA stands for no data available.

Table 6. Äverage ${ }^{137} \mathrm{Cs}_{,}{ }^{90} \mathrm{Sr}_{,}{ }^{239+240} \mathrm{Pu}$, and ${ }^{241} \mathrm{Am}$ soil concentrations in $\mathrm{pCi} / \mathrm{g}$ dry weight for soil profiles taken on Eniaetok Island (F33).

\begin{tabular}{|c|c|c|c|c|c|c|c|c|}
\hline \multirow{2}{*}{$\begin{array}{l}\text { Soil } \\
\text { depth } \\
\text { (cm) }\end{array}$} & \multicolumn{2}{|c|}{${ }^{137} \mathrm{Cs}$} & \multicolumn{2}{|c|}{${ }^{90} \mathrm{Sr}$} & \multicolumn{2}{|c|}{${ }^{239+240} \mathrm{Pu}$} & \multicolumn{2}{|c|}{${ }^{241} \mathrm{Am}$} \\
\hline & Median & Mean & Median & Mean & Median & Mean & Median & Mean \\
\hline $00-05$ & 21 & 21 & 5.4 & 6.6 & 6.6 & 7.2 & 5.5 & 5.1 \\
\hline $05-10$ & 8.0 & 9.1 & 8.1 & 9.2 & 3.0 & 3.3 & 0.88 & 1.7 \\
\hline $10-15$ & 3.3 & 4.4 & 3.4 & 6.1 & 2.0 & 2.6 & 0.61 & 0.70 \\
\hline $15-25$ & 1.3 & 1.5 & 2.1 & 3.3 & 0.31 & 1.8 & 0.11 & 0.16 \\
\hline $25-40$ & 0.49 & 0.75 & 3.1 & 5.9 & 0.059 & 0.056 & 0.027 & 0.041 \\
\hline $40-60$ & 0.50 & 1.6 & 2.7 & 2.4 & 0.089 & 0.17 & 0.067 & 0.11 \\
\hline $00-05$ & 21 & 21 & 5.4 & 6.6 & 6.6 & 7.2 & 5.5 & 5.1 \\
\hline $00-10$ & 13 & 15 & 6.8 & 7.9 & 2.6 & 2.6 & 2.8 & 3.1 \\
\hline $00-15$ & 10 & 11 & 5.9 & 7.3 & 2.1 & 2.6 & 1.6 & 2.1 \\
\hline $00-25$ & 7.2 & 7.2 & 4.4 & 5.7 & 1.4 & 2.3 & 0.76 & 0.78 \\
\hline $00-40$ & 4.8 & 4.8 & 4.0 & 5.8 & 0.87 & 1.4 & 0.37 & 0.48 \\
\hline $00-60$ & 2.7 & 2.5 & 4.0 & 4.7 & 0.71 & 1.0 & 0.28 & 0.35 \\
\hline
\end{tabular}

NOTE: Specific activity decay corrected to 1994. 
Table 7. Average ${ }^{137} \mathrm{Cs},{ }^{90} \mathrm{Sr}_{,}{ }^{239+240} \mathrm{Pu}$, and ${ }^{241} \mathrm{Am}$ soil concentrations in $\mathrm{pCi} / \mathrm{g}$ dry weight for soil profiles taken on Busch Island (F38).

\begin{tabular}{|c|c|c|c|c|c|c|c|c|}
\hline \multirow{2}{*}{$\begin{array}{l}\text { Soil } \\
\text { depth } \\
\text { (cm) }\end{array}$} & \multicolumn{2}{|c|}{${ }^{137} \mathrm{Cs}$} & \multicolumn{2}{|c|}{${ }^{90} \mathrm{Sr}$} & \multicolumn{2}{|c|}{$239+240 \mathrm{Pu}$} & \multicolumn{2}{|c|}{${ }^{241} \mathrm{Am}$} \\
\hline & Median & Mean & Median & $\overline{\text { Mean }}$ & Median & Mean & Median & Mean \\
\hline 00-05 & 12 & 27 & NDA & NDA & NDA & NDA & 5.4 & 6.9 \\
\hline $05-10$ & 22 & 23 & NDA & $\mathrm{NDA}$ & NDA & NDA & 6.0 & 6.0 \\
\hline $10-15$ & 16 & 15 & NDA & NDA & NDA & $\mathrm{NDA}$ & 2.6 & 3.1 \\
\hline $15-25$ & 6.0 & 4.9 & NDA & NDA & NDA & NDA & 0.51 & 0.69 \\
\hline $25-40$ & 1.4 & 2.0 & NDA & NDA & NDA & NDA & 0.43 & 0.43 \\
\hline $40-60$ & 0.0 & 0.0 & NDA & NDA & NDA & NDA & 0.0 & 0.0 \\
\hline $00-05$ & 12 & 27 & NDA & NDA & NDA & NDA & 5.4 & 6.9 \\
\hline $00-10$ & 23 & 25 & NDA & NDA & NDA & NDA & 3.5 & 3.5 \\
\hline $00-15$ & 22 & 22 & NDA & NDA & $\mathrm{NDA}$ & NDA & 3.5 & 3.5 \\
\hline $00-25$ & 15 & 15 & NDA & NDA & NDA & NDA & 2.4 & 2.4 \\
\hline $00-40$ & 9.9 & 10 & NDA & NDA & NDA & NDA & 1.3 & 1.3 \\
\hline $00-60$ & 0.0 & 0.0 & NDA & NDA & NDA & NDA & 0.0 & 0.0 \\
\hline
\end{tabular}

NOTE: Specific activity decay corrected to 1994.

NDA stands for no data available.

Table 8. Average ${ }^{137} \mathrm{Cs}^{90} \mathrm{Sr}^{239+240} \mathrm{Pu}$, and ${ }^{241} \mathrm{Am}$ soil concentrations in $\mathrm{pCi} / \mathrm{g}$ dry weight for soil profiles taken on Rongelap Island (F42).

\begin{tabular}{|c|c|c|c|c|c|c|c|c|}
\hline \multirow{2}{*}{$\begin{array}{l}\text { Soil } \\
\text { depth } \\
\text { (cm) }\end{array}$} & \multicolumn{2}{|c|}{${ }^{137} \mathrm{Cs}$} & \multicolumn{2}{|c|}{${ }^{90} \mathrm{Sr}$} & \multicolumn{2}{|c|}{$239+240 \mathrm{Pu}$} & \multicolumn{2}{|c|}{${ }^{241} \mathrm{Am}$} \\
\hline & Median & Mean & Median & Mean & Median & $\overline{\text { Mean }}$ & Median & Mean \\
\hline $00-05$ & 14 & 14 & 5.2 & 4.8 & 2.8 & 3.4 & 1.6 & 2.0 \\
\hline $05-10$ & 6.5 & 7.4 & 3.3 & 5.5 & 1.0 & 2.1 & 0.68 & 1.3 \\
\hline $10-15$ & 2.9 & 4.0 & 3.1 & 4.1 & 0.50 & 0.92 & 0.48 & 0.61 \\
\hline $15-25$ & 1.2 & 1.8 & 2.2 & 3.1 & 0.20 & 0.27 & 0.18 & 0.31 \\
\hline $25-40$ & 0.36 & 0.60 & 1.4 & 1.8 & 0.090 & 0.13 & 0.046 & 0.079 \\
\hline $40-60$ & 0.19 & 0.36 & 0.0 & 0.0 & 0.0 & 0.0 & 0.020 & 0.071 \\
\hline $00-05$ & 14 & 14 & 5.2 & 4.8 & 2.8 & 3.4 & 1.6 & 2.0 \\
\hline $00-10$ & 9.4 & 11 & 4.1 & 5.2 & 1.8 & 2.6 & 1.7 & 2.2 \\
\hline $00-15$ & 7.5 & 8.8 & 3.7 & 4.8 & 1.4 & 2.0 & 1.4 & 1.6 \\
\hline $00-25$ & 5.1 & 6.0 & 3.6 & 4.2 & 1.2 & 1.4 & 0.87 & 1.0 \\
\hline $00-40$ & 3.5 & 3.9 & 2.9 & 3.3 & 0.82 & 0.90 & 0.32 & 0.38 \\
\hline $00-60$ & 2.4 & 2.7 & 0.0 & 0.0 & 0.0 & 0.0 & 0.18 & 0.25 \\
\hline
\end{tabular}

NOTE: Specific activity decay corrected to 1994. 
Table 9. Average ${ }^{137} \mathrm{Cs},{ }^{90} \mathrm{Sr},{ }^{239+240} \mathrm{Pu}$, and ${ }^{241} \mathrm{Am}$ soil concentrations in $\mathrm{pCi} / \mathrm{g} \mathrm{dry}$ weight for soil profiles taken on Arbar Island (F43).

\begin{tabular}{|c|c|c|c|c|c|c|c|c|}
\hline \multirow{2}{*}{$\begin{array}{l}\text { Soil } \\
\text { depth } \\
\text { (cm) }\end{array}$} & \multicolumn{2}{|c|}{${ }^{137} \mathrm{Cs}$} & \multicolumn{2}{|c|}{${ }^{90} \mathrm{Sr}$} & \multicolumn{2}{|c|}{$239+240 \mathrm{Pu}$} & \multicolumn{2}{|c|}{${ }^{241} \mathrm{Am}$} \\
\hline & Median & Mean & Median & Mean & Median & Mean & Median & Mean \\
\hline $00-05$ & 10 & 14 & NDA & NDA & NDA & $\mathrm{NDA}$ & 3.5 & 7.0 \\
\hline $05-10$ & 7.3 & 11 & NDA & NDA & NDA & NDA & 0.95 & 2.2 \\
\hline $10-15$ & 2.4 & 6.6 & NDA & $\mathrm{NDA}$ & NDA & $\mathrm{NDA}$ & 2.7 & 2.7 \\
\hline $15-25$ & 1.5 & 3.3 & NDA & NDA & NDA & NDA & 2.9 & 2.9 \\
\hline $25-40$ & 1.0 & 0.80 & NDA & NDA & $\mathrm{NDA}$ & NDA & 0.0 & 0.0 \\
\hline $40-60$ & 0.31 & 0.47 & NDA & NDA & NDA & NDA & 0.0 & 0.0 \\
\hline $00-05$ & 10 & 14 & $\mathrm{NDA}$ & NDA & $\mathrm{NDA}$ & $\mathrm{NDA}$ & 3.5 & 7.0 \\
\hline $00-10$ & 10 & 13 & NDA & NDA & NDA & NDA & 2.7 & 4.1 \\
\hline $00-15$ & 7.3 & 11 & $\mathrm{NDA}$ & NDA & NDA & NDA & 2.7 & 2.7 \\
\hline $00-25$ & 5.3 & 8.0 & NDA & NDA & $\mathrm{NDA}$ & NDA & 3.5 & 3.5 \\
\hline $00-40$ & 3.7 & 5.5 & NDA & NDA & NDA & NDA & 0.0 & 0.0 \\
\hline $00-60$ & 2.3 & 2.4 & NDA & NDA & $\mathrm{NDA}$ & NDA & 0.0 & 0.0 \\
\hline
\end{tabular}

NOTE: Specific activity decay corrected to 1994.

NDA stands for no data available.

Table 10. Average ${ }^{137} \mathrm{CS}_{,}{ }^{90} \mathrm{Sr}_{1}{ }^{239+240} \mathrm{Pu}$, and ${ }^{241} \mathrm{Am}$ soil concentrations in $\mathrm{pCi} / \mathrm{g} \mathrm{dry}$ weight for soil profiles taken on Eniran Island (F46).

\begin{tabular}{|c|c|c|c|c|c|c|c|c|c|}
\hline \multirow{2}{*}{$\begin{array}{l}\text { Soil } \\
\text { depth } \\
\text { (cm) }\end{array}$} & \multicolumn{3}{|c|}{${ }^{137} \mathrm{Cs}$} & \multicolumn{2}{|c|}{${ }^{90} \mathrm{Sr}$} & \multicolumn{2}{|c|}{$239+240 \mathrm{Pu}$} & \multicolumn{2}{|c|}{${ }^{241} \mathrm{Am}$} \\
\hline & & Median & Mean & Median & Mean & Median & Mean & Median & Mean \\
\hline $00-05$ & & 13 & 13 & NDA & NDA & NDA & NDA & 5.0 & 4.8 \\
\hline $05-10$ & $r$ & 8.3 & 9.8 & NDA & NDA & NDA & NDA & 1.8 & 1.8 \\
\hline $10-15$ & & 3.3 & 3.9 & NDA & NDA & NDA & NDA & 0.60 & 0.60 \\
\hline $15-25$ & & 2.0 & 2.4 & NDA & $\mathrm{NDA}$ & NDA & NDA & 0.0 & 0.0 \\
\hline $25-40$ & & 0.34 & 0.91 & NDA & NDA & NDA & NDA & 0.0 & 0.0 \\
\hline \multirow[t]{2}{*}{$40-60$} & & 0.0 & 0.0 & NDA & $\mathrm{NDA}$ & NDA & NDA & 0.0 & 0.0 \\
\hline & & & & & & & NDA & & \\
\hline $00-05$ & & 13 & 13 & $\mathrm{NDA}$ & NDA & $\mathrm{NDA}$ & & 5.0 & 4.8 \\
\hline $00-10$ & & 12 & 12 & NDA & NDA & $\mathrm{NDA}$ & $\mathrm{NDA}$ & 2.3 & 2.3 \\
\hline $00-15$ & & 9.2 & 9.7 & NDA & NDA & NDA & NDA & 1.7 & 1.7 \\
\hline $00-25$ & & 6.6 & 6.9 & NDA & NDA & NDA & $\mathrm{NDA}$ & 0.0 & 0.0 \\
\hline $00-40$ & & 4.7 & 4.7 & NDA & NDA & NDA & NDA & 0.0 & 0.0 \\
\hline $00-60$ & & 0.0 & 0.0 & NDA & NDA & NDA & NDA & 0.0 & 0.0 \\
\hline
\end{tabular}

NOTE: Specific activity decay corrected to 1994.

NDA stands for no data available. 
Table 11. Average ${ }^{137} \mathrm{Cs},{ }^{90} \mathrm{Sr}_{,}{ }^{239+240} \mathrm{Pu}$, and ${ }^{241} \mathrm{Am}$ soil concentrations in $\mathrm{pCi} / \mathrm{g}$ dry weight for soil profiles taken on Tufa Island (F47).

\begin{tabular}{|c|c|c|c|c|c|c|c|c|}
\hline \multirow{2}{*}{$\begin{array}{l}\text { Soil } \\
\text { depth } \\
\text { (cm) }\end{array}$} & \multicolumn{2}{|c|}{${ }^{137} \mathrm{Cs}$} & \multicolumn{2}{|c|}{${ }^{90} \mathrm{Sr}$} & \multicolumn{2}{|c|}{$239+240 \mathrm{Pu}$} & \multicolumn{2}{|c|}{${ }^{241} \mathrm{Am}$} \\
\hline & Median & Mean & Median & Mean & Median & Mean & Median & Mean \\
\hline $00-05$ & 18 & 24 & NDA & NDA & NDA & NDA & 3.6 & 4.5 \\
\hline $05-10$ & 21 & 22 & $\mathrm{NDA}$ & NDA & NDA & NDA & 2.1 & 5.2 \\
\hline $10-15$ & 8.4 & 11 & NDA & NDA & NDA & NDA & 1.4 & 1.3 \\
\hline $15-25$ & 3.0 & 4.4 & NDA & NDA & NDA & NDA & 0.20 & 0.38 \\
\hline $25-40$ & 1.1 & 1.9 & NDA & NDA & NDA & NDA & 0.0 & 0.0 \\
\hline $40-60$ & 0.0 & 0.0 & NDA & $\mathrm{NDA}$ & NDA & NDA & 0.0 & 0.0 \\
\hline 00-05 & 18 & 24 & NDA & $\mathrm{NDA}$ & NDA & NDA & 3.6 & 4.5 \\
\hline $00-10$ & 17 & 23 & NDA & NDA & NDA & NDA & 3.5 & 4.7 \\
\hline $00-15$ & 15 & 19 & NDA & NDA & NDA & NDA & 3.6 & 3.9 \\
\hline $00-25$ & 9.8 & 13 & NDA & NDA & NDA & NDA & 3.3 & 2.9 \\
\hline $00-40$ & 6.9 & 8.8 & NDA & NDA & NDA & NDA & 0.0 & 0.0 \\
\hline $00-60$ & 0.0 & 0.0 & NDA & NDA & NDA & NDA & 0.0 & 0.0 \\
\hline
\end{tabular}

NOTE: Specific activity decay corrected to 1994.

NDA stands for no data available.

Table 12. Average ${ }^{137} \mathrm{Cs}_{,}{ }^{90} \mathrm{Sr},{ }^{239+240} \mathrm{Pu}$, and ${ }^{241} \mathrm{Am}$ soil concentrations in $\mathrm{pCi} / \mathrm{g}$ dry weight for soil profiles taken on Borukka Island (F49).

\begin{tabular}{|c|c|c|c|c|c|c|c|c|}
\hline \multirow{2}{*}{$\begin{array}{l}\text { Soil } \\
\text { depth } \\
\text { (cm) }\end{array}$} & \multicolumn{2}{|c|}{${ }^{137} \mathrm{Cs}$} & \multicolumn{2}{|c|}{${ }^{90} \mathrm{Sr}$} & \multicolumn{2}{|c|}{$239+240 \mathrm{Pu}$} & \multicolumn{2}{|c|}{${ }^{241} \mathrm{Am}$} \\
\hline & Median & Mean & Median & Mean & Median & Mean & Median & Mean \\
\hline $\begin{array}{l}00-05 \\
05-10 \\
10-15 \\
15-25 \\
25-40 \\
40-60\end{array}$ & $\begin{array}{l}22 \\
13 \\
7.2 \\
2.6 \\
0.84 \\
0.0\end{array}$ & $\begin{array}{l}25 \\
15 \\
9.1 \\
4.3 \\
1.4 \\
0.0\end{array}$ & $\begin{array}{c}12 \\
9.3 \\
6.2 \\
2.4 \\
2.5 \\
0.0\end{array}$ & $\begin{array}{c}12 \\
7.6 \\
5.6 \\
3.0 \\
2.5 \\
0.0\end{array}$ & $\begin{array}{l}5.3 \\
0.66 \\
0.25 \\
0.18 \\
0.069 \\
0.0\end{array}$ & $\begin{array}{l}6.9 \\
0.93 \\
0.29 \\
0.16 \\
0.069 \\
0.0\end{array}$ & $\begin{array}{l}3.2 \\
0.88 \\
0.61 \\
0.20 \\
0.035 \\
0.0\end{array}$ & $\begin{array}{l}4.5 \\
2.6 \\
0.76 \\
0.26 \\
0.092 \\
0.0\end{array}$ \\
\hline $\begin{array}{l}00-05 \\
00-10 \\
00-15 \\
00-25 \\
00-40 \\
00-60\end{array}$ & $\begin{array}{c}22 \\
17 \\
14 \\
9.5 \\
6.1 \\
0.0\end{array}$ & $\begin{array}{l}25 \\
20 \\
17 \\
12 \\
7.8 \\
0.0\end{array}$ & $\begin{array}{c}12 \\
10 \\
9.0 \\
6.0 \\
5.2 \\
0.0\end{array}$ & $\begin{array}{c}12 \\
9.6 \\
8.3 \\
6.2 \\
5.2 \\
0.0\end{array}$ & $\begin{array}{l}5.3 \\
4.3 \\
2.9 \\
1.8 \\
1.3 \\
0.0 \\
\end{array}$ & $\begin{array}{l}6.9 \\
4.1 \\
2.8 \\
1.7 \\
1.3 \\
0.0 \\
\end{array}$ & $\begin{array}{l}3.2 \\
2.2 \\
1.7 \\
0.82 \\
0.48 \\
0.0\end{array}$ & $\begin{array}{l}4.5 \\
3.3 \\
2.0 \\
1.3 \\
0.46 \\
0.0\end{array}$ \\
\hline
\end{tabular}

NOTE: Specific activity decay corrected to 1994. 


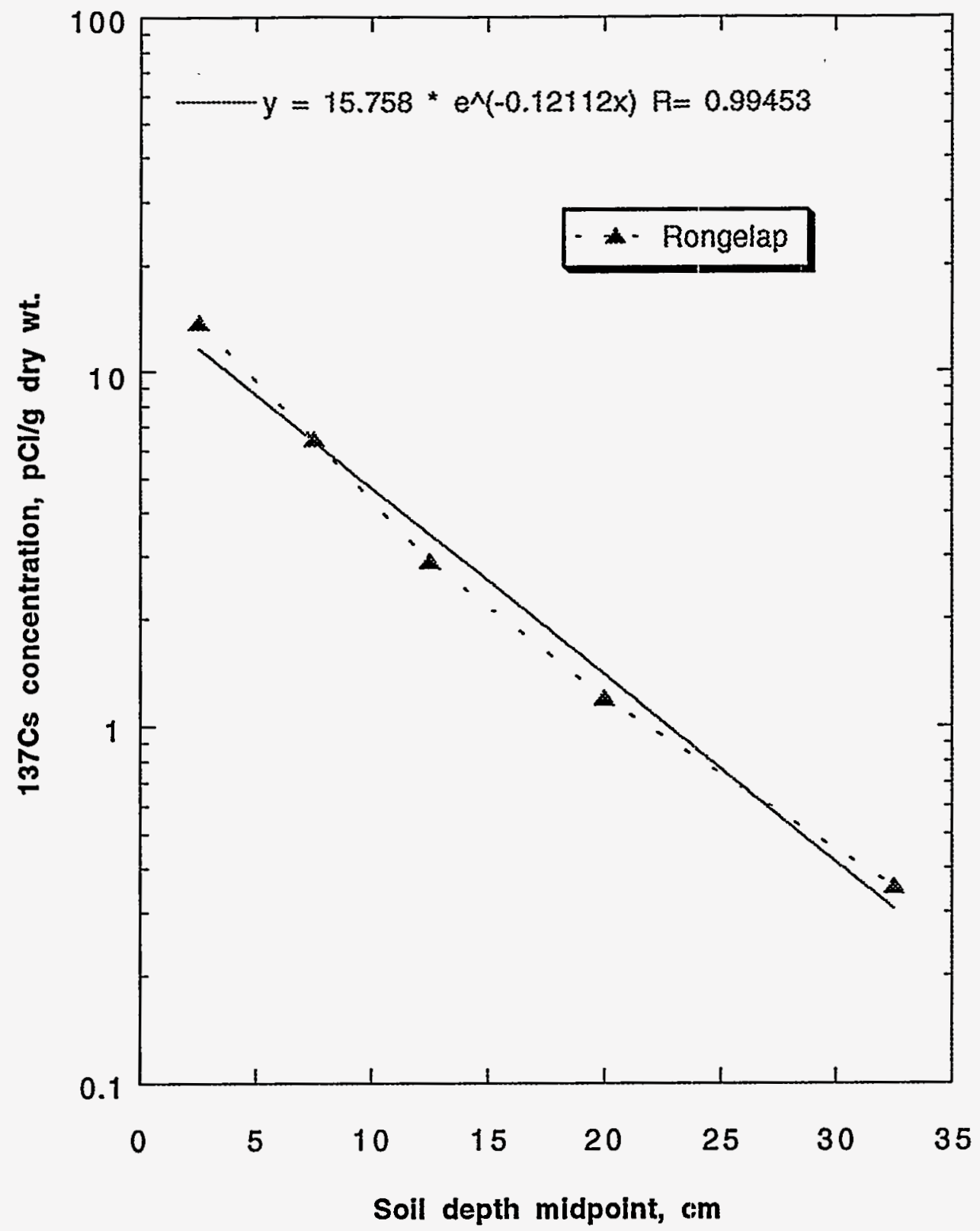

Figure 1. Rolngelap Island $-{ }^{137} \mathrm{Cs}$ Distribution in Soil. 


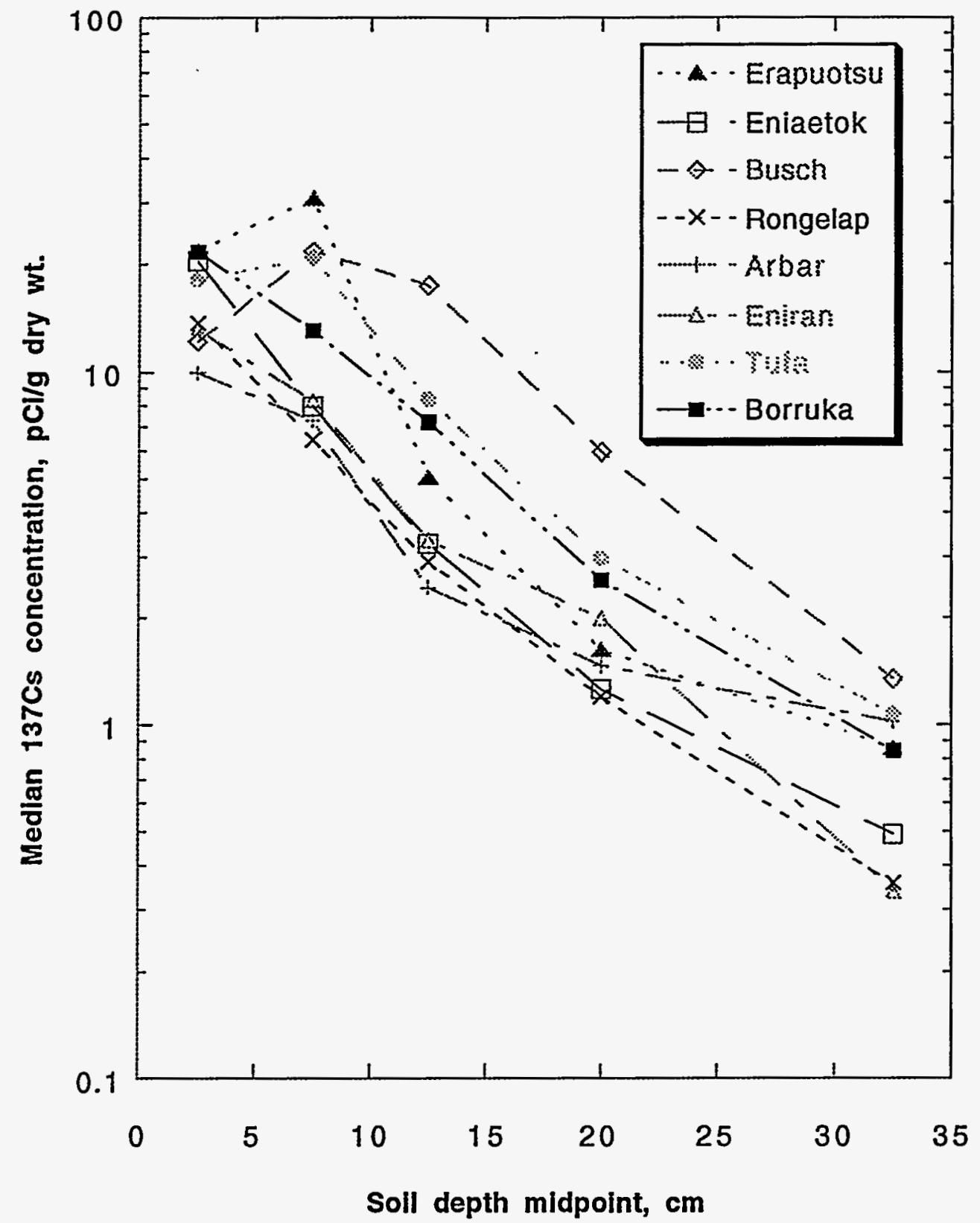

Figure 2. ${ }^{137} \mathrm{Cs}$ in soil at southern islands of Rongelap Atoll. 


\section{Appendix A}

Radionuclide concentration summary in vegetation samples collected during the 1978 NMIRS and from 1986 through 1991 in the southern islands of Rongelap Atoll. 
Table A-1. The ${ }^{137} \mathrm{Cs}$ radionuclide concentration summary for vegetation (food crops) collected from the southem islands of Rongelap Atoll during the 1978 NMIRS together with our recent trips in 1986 through 1991.

\begin{tabular}{|c|c|c|c|c|c|c|c|c|c|}
\hline \multirow{2}{*}{$\begin{array}{l}\text { Island } \\
\text { name }\end{array}$} & \multirow{2}{*}{$\begin{array}{l}\text { Island } \\
\text { code }\end{array}$} & \multirow[b]{2}{*}{$\mathrm{N}^{\mathbf{a}}$} & \multicolumn{5}{|c|}{ pCi g-1 wet wt } & \multirow{2}{*}{$\begin{array}{l}\text { Mean } \\
\text { of logs }\end{array}$} & \multirow{2}{*}{$\begin{array}{c}\text { SD } \\
\text { of logs }\end{array}$} \\
\hline & & & Minimum & Maximum & Median & Mean & SD & & \\
\hline \multicolumn{10}{|c|}{ Drinking Coconut Meat } \\
\hline $\begin{array}{l}\text { Erapuotsu } \\
\text { Eniaetok } \\
\text { Busch } \\
\text { Rongelap } \\
\text { Arbar } \\
\text { Eniran } \\
\text { Tufa } \\
\text { Borukka }\end{array}$ & $\begin{array}{l}32 f \\
33 f \\
38 f \\
42 f \\
43 f \\
46 f \\
47 f \\
49 f\end{array}$ & $\begin{array}{c}3 \\
7 \\
5 \\
200 \\
4 \\
4 \\
6 \\
5\end{array}$ & $\begin{array}{l}1.5 \times 10^{0} \\
1.7 \times 10^{-1} \\
5.6 \times 10^{-1} \\
2.9 \times 10^{-1} \\
4.9 \times 10^{-1} \\
4.7 \times 10^{-1} \\
3.7 \times 10^{-1} \\
6.3 \times 10^{-1}\end{array}$ & $\begin{array}{l}3.1 \times 10^{0} \\
7.9 \times 10^{0} \\
1.6 \times 10^{0} \\
9.8 \times 10^{0} \\
1.5 \times 10^{0} \\
1.9 \times 10^{0} \\
1.6 \times 10^{0} \\
2.5 \times 10^{0}\end{array}$ & $\begin{array}{l}2.0 \times 10^{0} \\
1.7 \times 10^{0} \\
1.1 \times 10^{0} \\
1.4 \times 10^{0} \\
8.8 \times 10^{-1} \\
9.8 \times 10^{-1} \\
7.4 \times 10^{-1} \\
1.2 \times 10^{0}\end{array}$ & $\begin{array}{l}2.2 \times 10^{0} \\
2.7 \times 10^{0} \\
1.1 \times 10^{0} \\
1.9 \times 10^{0} \\
9.3 \times 10^{-1} \\
1.1 \times 10^{0} \\
7.8 \times 10^{-1} \\
1.3 \times 10^{0}\end{array}$ & $\begin{array}{l}8.2 \times 10^{-1} \\
2.9 \times 10^{0} \\
3.8 \times 10^{-1} \\
1.4 \times 10^{0} \\
4.9 \times 10^{-1} \\
6.0 \times 10^{-1} \\
4.2 \times 10^{-1} \\
7.4 \times 10^{-1}\end{array}$ & $\begin{array}{c}7.4 \times 10^{-1} \\
3.1 \times 10^{-1} \\
3.6 \times 10^{-2} \\
4.0 \times 10^{-1} \\
-1.9 \times 10^{-1} \\
-4.0 \times 10^{-2} \\
-3.6 \times 10^{-1} \\
1.6 \times 10^{-1}\end{array}$ & $\begin{array}{l}3.7 \times 10^{-1} \\
1.4 \times 10^{0} \\
3.9 \times 10^{-1} \\
6.9 \times 10^{-1} \\
5.7 \times 10^{-1} \\
5.8 \times 10^{-1} \\
5.0 \times 10^{-1} \\
5.5 \times 10^{-1}\end{array}$ \\
\hline \multicolumn{10}{|c|}{ Drinking Coconut Juice } \\
\hline $\begin{array}{l}\text { Erapuotsu } \\
\text { Eniaetok } \\
\text { Busch } \\
\text { Rongelap } \\
\text { Arbar } \\
\text { Eniran } \\
\text { Tufa } \\
\text { Borukka }\end{array}$ & $\begin{array}{l}32 f \\
33 f \\
38 f \\
42 f \\
43 f \\
46 f \\
47 f \\
49 f\end{array}$ & $\begin{array}{c}3 \\
7 \\
5 \\
194 \\
4 \\
4 \\
7 \\
5\end{array}$ & $\begin{array}{l}4.0 \times 10^{-1} \\
1.8 \times 10^{-2} \\
1.6 \times 10^{-1} \\
9.2 \times 10^{-2} \\
5.0 \times 10^{-2} \\
1.9 \times 10^{-1} \\
7.9 \times 10^{-2} \\
8.8 \times 10^{-2}\end{array}$ & $\begin{array}{l}1.4 \times 10^{0} \\
1.8 \times 10^{0} \\
4.5 \times 10^{-1} \\
3.7 \times 10^{0} \\
3.0 \times 10^{-1} \\
7.7 \times 10^{-1} \\
3.5 \times 10^{-1} \\
1.2 \times 10^{0}\end{array}$ & $\begin{array}{l}7.9 \times 10^{-1} \\
3.2 \times 10^{-1} \\
2.8 \times 10^{-1} \\
6.2 \times 10^{-1} \\
1.7 \times 10^{-1} \\
4.7 \times 10^{-1} \\
2.1 \times 10^{-1} \\
3.6 \times 10^{-1}\end{array}$ & $\begin{array}{l}8.5 \times 10^{-1} \\
5.6 \times 10^{-1} \\
3.2 \times 10^{-1} \\
8.5 \times 10^{-1} \\
1.7 \times 10^{-1} \\
4.8 \times 10^{-1} \\
2.2 \times 10^{-1} \\
4.6 \times 10^{-1}\end{array}$ & $\begin{array}{l}4.8 \times 10^{-1} \\
6.1 \times 10^{-1} \\
1.2 \times 10^{-1} \\
6.7 \times 10^{-1} \\
1.1 \times 10^{-1} \\
2.5 \times 10^{-1} \\
1.0 \times 10^{-1} \\
4.2 \times 10^{-1}\end{array}$ & $\begin{array}{l}-2.8 \times 10^{-1} \\
-1.2 \times 10^{0} \\
-1.2 \times 10^{0} \\
-4.6 \times 10^{-1} \\
-2.0 \times 10^{0} \\
-8.6 \times 10^{-1} \\
-1.6 \times 10^{0} \\
-1.1 \times 10^{0}\end{array}$ & $\begin{array}{l}6.2 \times 10^{-1} \\
1.5 \times 10^{0} \\
4.1 \times 10^{-1} \\
8.0 \times 10^{-1} \\
7.7 \times 10^{-1} \\
6.0 \times 10^{-1} \\
5.6 \times 10^{-1} \\
9.4 \times 10^{-1}\end{array}$ \\
\hline \multicolumn{10}{|c|}{ Copra Meat } \\
\hline $\begin{array}{l}\text { Eniaetok } \\
\text { Rongelap } \\
\text { Arbar } \\
\text { Tufa } \\
\text { Borukka }\end{array}$ & $\begin{array}{l}33 f \\
42 f \\
43 f \\
47 f \\
49 f\end{array}$ & $\begin{array}{l}14 \\
72 \\
1 \\
1 \\
6\end{array}$ & $\begin{array}{l}6.1 \times 10^{-1} \\
8.4 \times 10^{-1} \\
8.2 \times 10^{-1} \\
1.1 \times 10^{0} \\
9.5 \times 10^{-1}\end{array}$ & $\begin{array}{l}7.2 \times 10^{0} \\
1.8 \times 10^{1} \\
8.2 \times 10^{-1} \\
1.1 \times 10^{0} \\
7.1 \times 10^{0}\end{array}$ & $\begin{array}{l}3.3 \times 10^{0} \\
2.6 \times 10^{0} \\
8.2 \times 10^{-1} \\
1.1 \times 10^{0} \\
2.0 \times 10^{0}\end{array}$ & $\begin{array}{l}3.4 \times 10^{0} \\
3.4 \times 10^{0} \\
8.2 \times 10^{-1} \\
1.1 \times 10^{0} \\
2.9 \times 10^{0}\end{array}$ & $\begin{array}{l}1.9 \times 10^{0} \\
2.7 \times 10^{0} \\
0.0 \times 10^{0} \\
0.0 \times 10^{0} \\
2.4 \times 10^{0}\end{array}$ & $\begin{array}{c}1.0 \times 10^{0} \\
1.0 \times 10^{0} \\
-1.9 \times 10^{-1} \\
7.8 \times 10^{-2} \\
7.9 \times 10^{-1}\end{array}$ & $\begin{array}{l}7.0 \times 10^{-1} \\
6.6 \times 10^{-1} \\
0.0 \times 10^{0} \\
0.0 \times 10^{0} \\
7.9 \times 10^{-1}\end{array}$ \\
\hline \multicolumn{10}{|c|}{ Copra Juice } \\
\hline $\begin{array}{l}\text { Eniaetok } \\
\text { Rongelap } \\
\text { Arbar } \\
\text { Tufa } \\
\text { Borukka }\end{array}$ & $\begin{array}{l}33 f \\
42 f \\
43 f \\
47 f \\
49 f\end{array}$ & $\begin{array}{l}14 \\
70 \\
1 \\
1 \\
6\end{array}$ & $\begin{array}{l}5.2 \times 10^{-1} \\
2.5 \times 10^{-1} \\
1.1 \times 10^{0} \\
8.0 \times 10^{-1} \\
3.6 \times 10^{-1}\end{array}$ & $\begin{array}{l}5.6 \times 10^{0} \\
6.4 \times 10^{0} \\
1.1 \times 10^{0} \\
8.0 \times 10^{-1} \\
5.1 \times 10^{0}\end{array}$ & $\begin{array}{l}2.4 \times 10^{0} \\
1.4 \times 10^{0} \\
1.1 \times 10^{0} \\
8.0 \times 10^{-1} \\
1.2 \times 10^{0}\end{array}$ & $\begin{array}{l}2.5 \times 10^{0} \\
1.9 \times 10^{0} \\
1.1 \times 10^{0} \\
8.0 \times 10^{-1} \\
2.1 \times 10^{0}\end{array}$ & $\begin{array}{l}1.6 \times 10^{0} \\
1.5 \times 10^{0} \\
0.0 \times 10^{0} \\
0.0 \times 10^{0} \\
2.0 \times 10^{0}\end{array}$ & $\begin{array}{c}6.5 \times 10^{-1} \\
3.4 \times 10^{-1} \\
6.6 \times 10^{-2} \\
-2.2 \times 10^{-1} \\
3.3 \times 10^{-1}\end{array}$ & $\begin{array}{l}8.0 \times 10^{-1} \\
7.8 \times 10^{-1} \\
0.0 \times 10^{0} \\
0.0 \times 10^{0} \\
1.0 \times 10^{0}\end{array}$ \\
\hline \multicolumn{10}{|c|}{ Pandanus } \\
\hline $\begin{array}{l}\text { Erapuotsu } \\
\text { Eniaetok } \\
\text { Busch } \\
\text { Rongelap } \\
\text { Arbar } \\
\text { Tufa } \\
\text { Borukka }\end{array}$ & $\begin{array}{l}32 f \\
33 f \\
38 f \\
42 f \\
43 f \\
47 f \\
49 f\end{array}$ & $\begin{array}{c}3 \\
15 \\
2 \\
78 \\
4 \\
2 \\
9\end{array}$ & $\begin{array}{c}1.4 \times 10^{0} \\
5.3 \times 10^{-1} \\
5.6 \times 10^{-1} \\
5.1 \times 10^{-1} \\
5.7 \times 10^{-1} \\
3.4 \times 10^{0} \\
2.8 \times 10^{-1}\end{array}$ & $\begin{array}{l}4.0 \times 10^{0} \\
1.4 \times 10^{1} \\
1.1 \times 10^{0} \\
3.4 \times 10^{1} \\
8.9 \times 10^{0} \\
4.7 \times 10^{0} \\
2.9 \times 10^{0}\end{array}$ & $\begin{array}{c}2.0 \times 10^{0} \\
2.2 \times 10^{0} \\
8.3 \times 10^{-1} \\
5.9 \times 10^{0} \\
1.4 \times 10^{0} \\
4.0 \times 10^{0} \\
1.0 \times 10^{0}\end{array}$ & $\begin{array}{c}2.4 \times 10^{0} \\
4.0 \times 10^{0} \\
8.3 \times 10^{-1} \\
7.6 \times 10^{0} \\
3.1 \times 10^{0} \\
4.0 \times 10^{0} \\
1.4 \times 10^{0}\end{array}$ & $\begin{array}{l}1.4 \times 10^{0} \\
4.7 \times 10^{0} \\
3.8 \times 10^{-1} \\
6.5 \times 10^{0} \\
3.9 \times 10^{0} \\
9.4 \times 10^{-1} \\
9.5 \times 10^{-1}\end{array}$ & $\begin{array}{c}7.9 \times 10^{-1} \\
8.0 \times 10^{-1} \\
-2.5 \times 10^{-1} \\
1.7 \times 10^{0} \\
5.7 \times 10^{-1} \\
1.4 \times 10^{0} \\
6.7 \times 10^{-2}\end{array}$ & $\begin{array}{r}5.5 \times 10^{-1} \\
1.1 \times 10^{0} \\
4.8 \times 10^{-1} \\
8.3 \times 10^{-1} \\
1.2 \times 10^{0} \\
2.4 \times 10^{-1} \\
7.9 \times 10^{-1}\end{array}$ \\
\hline
\end{tabular}


Table A-1. The ${ }^{137} \mathrm{Cs}$ radionuclide concentration summary for vegetation (food crops) collected from the southem islands of Rongelap Atoll during the 1978 NMIRS together with our recent trips in 1986 through 1991 (continued).

\begin{tabular}{|c|c|c|c|c|c|c|c|c|c|}
\hline \multirow{2}{*}{$\begin{array}{l}\text { Island } \\
\text { name }\end{array}$} & \multirow{2}{*}{$\begin{array}{l}\text { Island } \\
\text { code }\end{array}$} & \multirow[b]{2}{*}{$\mathrm{N}^{\mathrm{a}}$} & \multicolumn{5}{|c|}{ pCi g ${ }^{-1}$ wet wt } & \multirow{2}{*}{$\begin{array}{l}\text { Mean } \\
\text { of logs }\end{array}$} & \multirow{2}{*}{$\begin{array}{c}\text { SD } \\
\text { of logs } \\
\end{array}$} \\
\hline & & & Minimum & Maximum & Median & Mean & SD & & \\
\hline \multicolumn{10}{|c|}{ Breadfruit Meat } \\
\hline Rongelap & $42 f$ & 30 & $1.0 \times 10^{0}$ & $7.0 \times 10^{0}$ & $3.0 \times 10^{0}$ & $3.2 \times 10^{0}$ & $1.5 \times 10^{0}$ & $1.0 \times 10^{0}$ & $4.9 \times 10^{-1}$ \\
\hline \multicolumn{10}{|c|}{ Tacca Meat } \\
\hline $\begin{array}{l}\text { Busch } \\
\text { Rongelap } \\
\text { Tufa } \\
\text { Borukka }\end{array}$ & $\begin{array}{l}38 f \\
42 f \\
47 f \\
49 f\end{array}$ & $\begin{array}{l}1 \\
5 \\
2 \\
4\end{array}$ & $\begin{array}{c}2.3 \times 10^{0} \\
1.0 \times 10^{0} \\
5.2 \times 10^{-1} \\
5.2 \times 10^{-1}\end{array}$ & $\begin{array}{l}2.3 \times 10^{0} \\
1.5 \times 10^{1} \\
2.7 \times 10^{0} \\
2.1 \times 10^{0}\end{array}$ & $\begin{array}{l}2.3 \times 10^{0} \\
4.3 \times 10^{0} \\
1.6 \times 10^{0} \\
1.2 \times 10^{0}\end{array}$ & $\begin{array}{l}2.3 \times 10^{0} \\
5.7 \times 10^{0} \\
1.6 \times 10^{0} \\
1.2 \times 10^{0}\end{array}$ & $\begin{array}{l}0.0 \times 10^{0} \\
5.4 \times 10^{0} \\
1.5 \times 10^{0} \\
7.5 \times 10^{-1}\end{array}$ & $\begin{array}{l}8.4 \times 10^{-1} \\
1.4 \times 10^{0} \\
1.7 \times 10^{-1} \\
4.4 \times 10^{-2}\end{array}$ & $\begin{array}{l}0.0 \times 10^{0} \\
9.6 \times 10^{-1} \\
1.2 \times 10^{0} \\
6.7 \times 10^{-1}\end{array}$ \\
\hline \multicolumn{10}{|c|}{ Lime Meat } \\
\hline Rongelap & $42 \mathrm{f}$ & 9 & $1.2 \times 10^{0}$ & $2.0 \times 10^{0}$ & $1.5 \times 10^{0}$ & $1.6 \times 10^{0}$ & $2.5 \times 10^{-1}$ & $4.4 \times 10^{-1}$ & $1.6 \times 10^{-1}$ \\
\hline \multicolumn{10}{|c|}{ Squash Meat } \\
\hline Rongelap & $42 f$ & 2 & $3.7 \times 10^{0}$ & $7.8 \times 10^{0}$ & $5.8 \times 10^{0}$ & $5.8 \times 10^{0}$ & $2.9 \times 10^{0}$ & $1.7 \times 10^{0}$ & $5.2 \times 10^{-1}$ \\
\hline
\end{tabular}

NOTE: Specific activity is decay corrected to 1994 .

$\mathrm{N}^{\mathrm{a}}$ stands for number of individual samples. 
Table A-2. The ${ }^{137} \mathrm{Cs}$ radionuclide concentration summary for vegetation (indicator species) collected from the southem islands of Rongelap Atoll during the 1978 NMIRS together with our recent trips in 1986 through 1991.

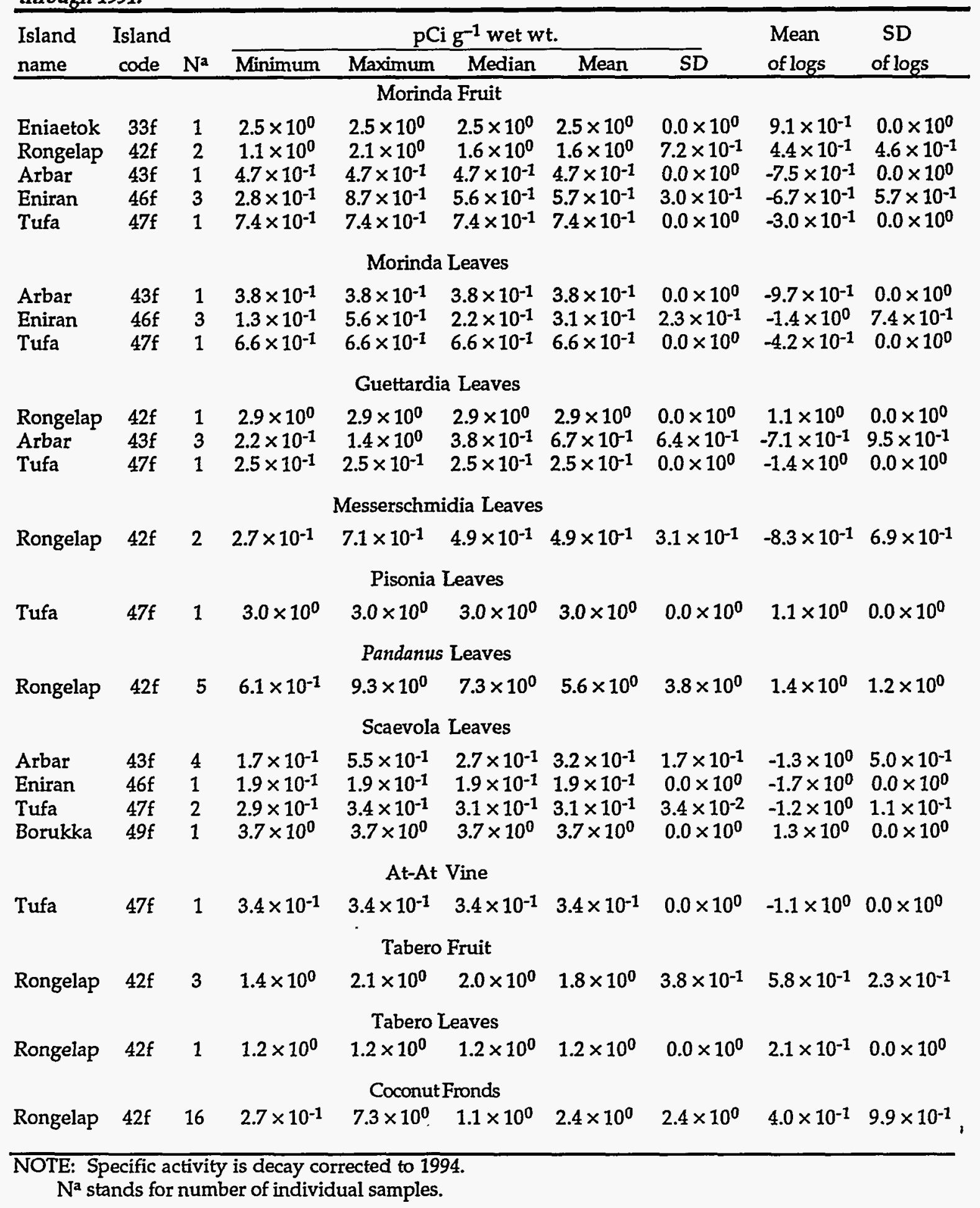


Appendix B

Radionuclide concentration summary in animal samples collected during the 1978 NMIRS and from 1986 through 1991 in the southern islands of Rongelap Atoll. 
Table B-1. The ${ }^{137} \mathrm{Cs}$ radionuclide concentration summary for animals (food source) collected from the southem islands of Rongelap Atoll during the 1978 NMIRS together with our recent trips in 1986 through 1991.

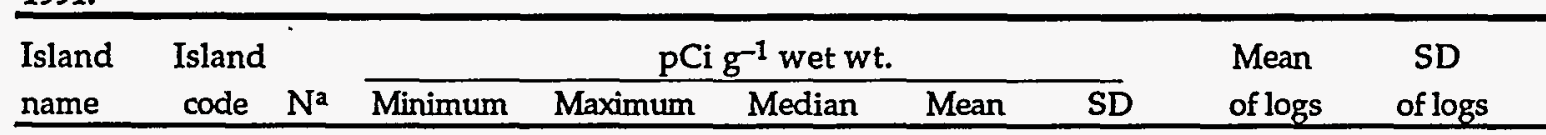

\section{Coconut Crab Muscle}

$\begin{array}{llllllllll}\text { Erapuotsu } & 32 \mathrm{f} & 1 & 4.9 \times 10^{0} & 4.9 \times 10^{0} & 4.88 \times 10^{0} & 4.9 \times 10^{0} & 0.0 \times 10^{0} & 1.6 \times 10^{0} & 0.0 \times 10^{0} \\ \text { Busch } & 38 \mathrm{f} & 1 & 1.4 \times 10^{0} & 1.4 \times 10^{0} & 1.39 \times 10^{0} & 1.4 \times 10^{0} & 0.0 \times 10^{0} & 3.3 \times 10^{-1} & 0.0 \times 10^{0} \\ \text { Arbar } & 43 \mathrm{f} & 2 & 2.3 \times 10^{0} & 2.6 \times 10^{0} & 2.5 \times 10^{0} & 2.5 \times 10^{0} & 1.9 \times 10^{-1} & 9.0 \times 10^{-1} & 7.8 \times 10^{-2} \\ \text { Eniran } & 46 \mathrm{f} & 2 & 1.1 \times 10^{0} & 2.0 \times 10^{0} & 1.5 \times 10^{0} & 1.5 \times 10^{0} & 6.5 \times 10^{-1} & 3.8 \times 10^{-1} & 4.4 \times 10^{-1} \\ \text { Tufa } & 47 \mathrm{f} & 2 & 1.2 \times 10^{0} & 2.0 \times 10^{0} & 1.6 \times 10^{0} & 1.6 \times 10^{0} & 6.1 \times 10^{-1} & 4.2 \times 10^{-1} & 3.9 \times 10^{-1} \\ \text { Borukka } & 49 \mathrm{f} & 1 & 2.1 \times 10^{0} & 2.1 \times 10^{0} & 2.1 \times 10^{0} & 2.1 \times 10^{0} & 0.0 \times 10^{0} & 7.3 \times 10^{-1} & 0.0 \times 10^{0}\end{array}$

Coconut Crab Hepatopancreas

$\begin{array}{llllllllll}\text { Erapuotsu } & 32 \mathrm{f} & 1 & 3.4 \times 10^{0} & 3.4 \times 10^{0} & 3.4 \times 10^{0} & 3.4 \times 10^{0} & 0.0 \times 10^{0} & 1.2 \times 10^{0} & 0.0 \times 10^{0} \\ \text { Busch } & 38 \mathrm{f} & 1 & 9.8 \times 10^{-1} & 9.8 \times 10^{-1} & 9.8 \times 10^{-1} & 9.8 \times 10^{-1} & 0.0 \times 10^{0} & -2.3 \times 10^{-2} & 0.0 \times 10^{0} \\ \text { Eniran } & 46 \mathrm{f} & 2 & 9.6 \times 10^{-1} & 1.3 \times 10^{0} & 1.1 \times 10^{0} & 1.1 \times 10^{0} & 2.2 \times 10^{-1} & 1.1 \times 10^{-1} & 2.0 \times 10^{-1} \\ \text { Tufa } & 47 \mathrm{f} & 2 & 9.0 \times 10^{-1} & 1.3 \times 10^{0} & 1.1 \times 10^{0} & 1.1 \times 10^{0} & 2.2 \times 10^{-1} & 7.0 \times 10^{-2} & 2.5 \times 10^{-1} \\ \text { Borukka } & 49 \mathrm{f} & 1 & 1.9 \times 10^{0} & 1.9 \times 10^{0} & 1.9 \times 10^{0} & 1.9 \times 10^{0} & 0.0 \times 10^{0} & 6.3 \times 10^{-1} & 0.0 \times 10^{0}\end{array}$

Pig Heart

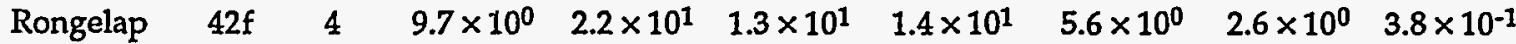

Pig Liver

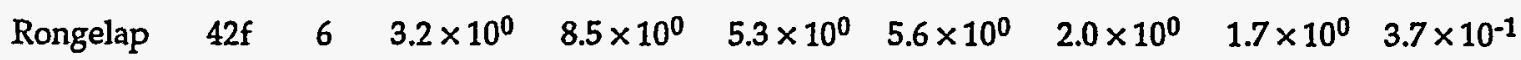

Pig Muscle

$\begin{array}{lllllllll}\text { Rongelap } \quad 42 \mathrm{f} & 7 & 5.3 \times 10^{0} & 2.9 \times 10^{1} & 1.3 \times 10^{1} & 1.4 \times 10^{1} & 7.8 \times 10^{0} & 2.5 \times 10^{0} & 5.6 \times 10^{-1}\end{array}$ NOTE: Specific activity is decay corrected to 1994.

$\mathrm{N}^{2}$ stands for number of individual samples. 
Appendix C

Radionuclide concentration summary of all soil profile samples collected during the 1978 NMIRS and from 1986 through 1991 in the southern islands of Rongelap Atoll. 
Table C-1. Cesium-137 radionuclide concentration summary for all soil profiles taken from our most recent trip in 1991 on Erapuotsu Island (F32).

\begin{tabular}{|c|c|c|c|c|c|c|c|c|}
\hline \multirow{3}{*}{$\begin{array}{r}\text { Soil } \\
\text { depth } \\
(\mathrm{cm})\end{array}$} & \multirow[b]{3}{*}{$\mathrm{Na}^{\mathrm{a}}$} & \multirow{2}{*}{\multicolumn{5}{|c|}{ pCi g ${ }^{-1}$ dry wt }} & \multirow{3}{*}{$\begin{array}{l}\text { Mean } \\
\text { of logs }\end{array}$} & \multirow{3}{*}{$\begin{array}{c}S D \\
\text { of logs } \\
\end{array}$} \\
\hline & & & & & & & & \\
\hline & & Minimum & Maximum & Median & Mean & SD & & \\
\hline $0-05$ & 3 & $7.3 \times 10^{0}$ & $4.1 \times 10^{1}$ & $2.2 \times 10^{1}$ & $2.3 \times 10^{1}$ & $1.7 \times 10^{1}$ & $2.9 \times 10^{0}$ & $8.8 \times 10^{-1}$ \\
\hline $05-10$ & 3 & $3.0 \times 10^{1}$ & $4.3 \times 10^{1}$ & $3.1 \times 10^{1}$ & $3.5 \times 10^{1}$ & $7.4 \times 10^{0}$ & $3.5 \times 10^{0}$ & $2.0 \times 10^{-1}$ \\
\hline $10-15$ & 3 & $2.6 \times 10^{0}$ & $1.7 \times 10^{1}$ & $5.0 \times 10^{0}$ & $8.1 \times 10^{0}$ & $7.6 \times 10^{0}$ & $1.8 \times 10^{0}$ & $9.5 \times 10^{-1}$ \\
\hline $15-25$ & 3 & $7.0 \times 10^{-1}$ & $1.9 \times 10^{0}$ & $1.6 \times 10^{0}$ & $1.4 \times 10^{0}$ & $6.3 \times 10^{1}$ & $2.6 \times 10^{-1}$ & $5.4 \times 10^{-1}$ \\
\hline $25-40$ & 3 & $8.3 \times 10^{-1}$ & $1.2 \times 10^{0}$ & $8.6 \times 10^{-1}$ & $9.6 \times 10^{-1}$ & $1.9 \times 10^{-1}$ & $-5.8 \times 10^{-2}$ & $1.9 \times 10^{-1}$ \\
\hline $40-60$ & 0 & $0.0 \times 10^{0}$ & $0.0 \times 10^{0}$ & $0.0 \times 10^{0}$ & $0.0 \times 10^{0}$ & $0.0 \times 10^{0}$ & $0.0 \times 10^{0}$ & $0.0 \times 10^{0}$ \\
\hline $0-05$ & 3 & $7.3 \times 10^{0}$ & $4.1 \times 10^{1}$ & $2.2 \times 10^{1}$ & $2.3 \times 10^{1}$ & $1.7 \times 10^{1}$ & $2.9 \times 10^{0}$ & $8.8 \times 10^{-1}$ \\
\hline $0-10$ & 3 & $1.9 \times 10^{1}$ & $4.2 \times 10^{1}$ & $2.6 \times 10^{1}$ & $2.9 \times 10^{1}$ & $1.2 \times 10^{1}$ & $3.3 \times 10^{0}$ & $4.1 \times 10^{-1}$ \\
\hline $0-15$ & 3 & $1.4 \times 10^{1}$ & $3.4 \times 10^{1}$ & $1.8 \times 10^{1}$ & $2.2 \times 10^{1}$ & $1.0 \times 10^{1}$ & $3.0 \times 10^{0}$ & $4.5 \times 10^{-1}$ \\
\hline $0-25$ & 3 & $9.1 \times 10^{0}$ & $2.1 \times 10^{1}$ & $1.1 \times 10^{1}$ & $1.4 \times 10^{1}$ & $6.3 \times 10^{0}$ & $2.6 \times 10^{0}$ & $4.3 \times 10^{-1}$ \\
\hline $0-40$ & 3 & $6.1 \times 10^{0}$ & $1.3 \times 10^{1}$ & $7.4 \times 10^{0}$ & $9.0 \times 10^{0}$ & $3.9 \times 10^{0}$ & $2.1 \times 10^{0}$ & $4.1 \times 10^{-1}$ \\
\hline $0-60$ & 0 & $0.0 \times 10^{0}$ & $0.0 \times 10^{0}$ & $0.0 \times 10^{0}$ & $0.0 \times 10^{0}$ & $0.0 \times 10^{0}$ & $0.0 \times 10^{0}$ & $0.0 \times 10^{0}$ \\
\hline
\end{tabular}

NOTE: Specific activity is decay corrected to 1994.

$\mathrm{N}^{\mathrm{a}}$ stands for number of individual samples. 
Table C-2. Cesium-137 radionuclide concentration summary for all soil profiles taken from the 1978 NMIRS together with our most recent trip in 1989 on Eniaetok Island (F33).

\begin{tabular}{|c|c|c|c|c|c|c|c|c|}
\hline \multirow{2}{*}{$\begin{array}{r}\text { Soil } \\
\text { depth } \\
(\mathrm{cm}) \\
\end{array}$} & \multirow[b]{2}{*}{$\mathrm{Na}$} & \multicolumn{5}{|c|}{$\mathrm{pCig}^{-1}$ dry wt } & \multirow{2}{*}{$\begin{array}{l}\text { Mean } \\
\text { of logs }\end{array}$} & \multirow{2}{*}{$\begin{array}{r}\text { SD } \\
\text { of logs } \\
\end{array}$} \\
\hline & & Minimum & Maximum & Median & Mean & SD & & \\
\hline $0-05$ & 21 & $6.4 \times 10^{-2}$ & $5.7 \times 10^{1}$ & $2.1 \times 10^{1}$ & $2.1 \times 10^{1}$ & $1.7 \times 10^{1}$ & $2.3 \times 10^{0}$ & $1.8 \times 10^{0}$ \\
\hline $05-10$ & 21 & $2.8 \times 10^{-2}$ & $2.5 \times 10^{1}$ & $8.0 \times 10^{0}$ & $9.1 \times 10^{0}$ & $6.4 \times 10^{0}$ & $1.8 \times 10^{0}$ & $1.4 \times 10^{0}$ \\
\hline $10-15$ & 20 & $2.5 \times 10^{-2}$ & $1.4 \times 10^{1}$ & $3.3 \times 10^{0}$ & $4.4 \times 10^{0}$ & $3.4 \times 10^{0}$ & $1.1 \times 10^{0}$ & $1.3 \times 10^{0}$ \\
\hline $15-25$ & 21 & $4.3 \times 10^{-2}$ & $3.2 \times 10^{0}$ & $1.3 \times 10^{0}$ & $1.5 \times 10^{0}$ & $9.4 \times 10^{-1}$ & $7.6 \times 10^{-2}$ & $1.0 \times 10^{0}$ \\
\hline $25-40$ & 21 & $6.0 \times 10^{-2}$ & $2.4 \times 10^{0}$ & $4.9 \times 10^{-1}$ & $7.5 \times 10^{-1}$ & $6.1 \times 10^{-1}$ & $-6.5 \times 10^{-1}$ & $9.7 \times 10^{-1}$ \\
\hline $40-60$ & 6 & $2.3 \times 10^{-1}$ & $6.8 \times 10^{0}$ & $5.0 \times 10^{-1}$ & $1.6 \times 10^{0}$ & $2.6 \times 10^{0}$ & $-3.3 \times 10^{-1}$ & $1.2 \times 10^{0}$ \\
\hline $0-05$ & 21 & $6.4 \times 10^{-2}$ & $5.7 \times 10^{1}$ & $2.1 \times 10^{1}$ & $2.1 \times 10^{1}$ & $1.7 \times 10^{1}$ & $2.3 \times 10^{0}$ & $1.8 \times 10^{0}$ \\
\hline $0-10$ & 21 & $4.6 \times 10^{-2}$ & $3.5 \times 10^{1}$ & $1.3 \times 10^{1}$ & $1.5 \times 10^{1}$ & $1.0 \times 10^{1}$ & $2.2 \times 10^{0}$ & $1.5 \times 10^{0}$ \\
\hline $0-15$ & 20 & $4.0 \times 10^{-2}$ & $2.5 \times 10^{1}$ & $1.0 \times 10^{1}$ & $1.1 \times 10^{1}$ & $7.3 \times 10^{0}$ & $2.0 \times 10^{0}$ & $1.4 \times 10^{0}$ \\
\hline $0-25$ & 20 & $4.1 \times 10^{-2}$ & $1.5 \times 10^{1}$ & $7.2 \times 10^{0}$ & $7.2 \times 10^{0}$ & $4.5 \times 10^{0}$ & $1.6 \times 10^{0}$ & $1.3 \times 10^{0}$ \\
\hline $0-40$ & 20 & $5.0 \times 10^{-2}$ & $9.7 \times 10^{0}$ & $4.8 \times 10^{0}$ & $4.8 \times 10^{0}$ & $2.9 \times 10^{0}$ & $1.2 \times 10^{0}$ & $1.2 \times 10^{0}$ \\
\hline $0-60$ & 6 & $9.9 \times 10^{-1}$ & $4.6 \times 10^{0}$ & $2.7 \times 10^{0}$ & $2.5 \times 10^{0}$ & $1.4 \times 10^{0}$ & $7.9 \times 10^{-1}$ & $6.1 \times 10^{-1}$ \\
\hline
\end{tabular}

NOTE: Specific activity is decay corrected to 1994.

$\mathrm{N}^{\mathrm{a}}$ stands for number of individual samples. 
Table C-3. Cesium-137 radionuclide concentration summary for all soil profiles taken from our most recent trip in 1991 on Busch Island (F38).

\begin{tabular}{|c|c|c|c|c|c|c|c|c|}
\hline \multirow{2}{*}{$\begin{array}{l}\text { Soil } \\
\text { depth } \\
(\mathrm{cm})\end{array}$} & \multirow[b]{2}{*}{$\mathrm{Na}^{\mathrm{a}}$} & \multicolumn{4}{|c|}{ pCig $^{-1}$ dry wt } & & \multirow{2}{*}{$\begin{array}{l}\text { Mean } \\
\text { of logs }\end{array}$} & \multirow{2}{*}{$\begin{array}{c}S D \\
\text { of logs }\end{array}$} \\
\hline & & Minimum & Maximum & Median & Mean & $\mathrm{SD}$ & & \\
\hline $0-05$ & 5 & $2.2 \times 10^{0}$ & $8.5 \times 10^{1}$ & $1.2 \times 10^{1}$ & $2.7 \times 10^{1}$ & $3.4 \times 10^{1}$ & $2.6 \times 10^{0}$ & $1.4 \times 10^{0}$ \\
\hline $05-10$ & 5 & $1.1 \times 10^{1}$ & $3.4 \times 10^{1}$ & $2.2 \times 10^{1}$ & $2.3 \times 10^{1}$ & $1.1 \times 10^{1}$ & $3.0 \times 10^{0}$ & $5.3 \times 10^{-1}$ \\
\hline $10-15$ & 5 & $6.8 \times 10^{0}$ & $2.2 \times 10^{1}$ & $1.6 \times 10^{1}$ & $1.5 \times 10^{1}$ & $6.7 \times 10^{0}$ & $2.6 \times 10^{0}$ & $5.3 \times 10^{-1}$ \\
\hline $15-25$ & 5 & $7.5 \times 10^{-1}$ & $8.1 \times 10^{0}$ & $6.0 \times 10^{0}$ & $4.9 \times 10^{0}$ & $3.0 \times 10^{0}$ & $1.3 \times 10^{0}$ & $9.6 \times 10^{-1}$ \\
\hline $25-40$ & 5 & $3.1 \times 10^{-1}$ & $4.0 \times 10^{0}$ & $1.4 \times 10^{0}$ & $2.0 \times 10^{0}$ & $1.7 \times 10^{0}$ & $2.6 \times 10^{-1}$ & $1.1 \times 10^{0}$ \\
\hline $40-60$ & 0 & $0.0 \times 10^{0}$ & $0.0 \times 10^{0}$ & $0.0 \times 10^{0}$ & $0.0 \times 10^{0}$ & $0.0 \times 10^{0}$ & $0.0 \times 10^{0}$ & $0.0 \times 10^{0}$ \\
\hline $0-05$ & 5 & $2.2 \times 10^{0}$ & $8.5 \times 10^{1}$ & $1.2 \times 10^{1}$ & $2.7 \times 10^{1}$ & $3.4 \times 10^{1}$ & $2.6 \times 10^{0}$ & $1.4 \times 10^{0}$ \\
\hline $0-10$ & 5 & $6.8 \times 10^{0}$ & $5.3 \times 10^{1}$ & $2.3 \times 10^{1}$ & $2.5 \times 10^{1}$ & $1.9 \times 10^{1}$ & $3.0 \times 10^{0}$ & $8.4 \times 10^{-1}$ \\
\hline $0-15$ & 5 & $1.2 \times 10^{1}$ & $3.8 \times 10^{1}$ & $2.2 \times 10^{1}$ & $2.2 \times 10^{1}$ & $1.1 \times 10^{1}$ & $3.0 \times 10^{0}$ & $4.9 \times 10^{-1}$ \\
\hline $0-25$ & 5 & $9.5 \times 10^{0}$ & $2.3 \times 10^{1}$ & $1.5 \times 10^{1}$ & $1.5 \times 10^{1}$ & $5.4 \times 10^{0}$ & $2.7 \times 10^{0}$ & $3.5 \times 10^{-1}$ \\
\hline $0-40$ & 5 & $7.3 \times 10^{0}$ & $1.5 \times 10^{1}$ & $9.9 \times 10^{0}$ & $1.0 \times 10^{1}$ & $2.9 \times 10^{0}$ & $2.3 \times 10^{0}$ & $2.7 \times 10^{-1}$ \\
\hline $0-60$ & 0 & $0.0 \times 10^{0}$ & $0.0 \times 10^{0}$ & $0.0 \times 10^{0}$ & $0.0 \times 10^{0}$ & $0.0 \times 10^{0}$ & $0.0 \times 10^{0}$ & $0.0 \times 10^{0}$ \\
\hline
\end{tabular}

NOTE: Specific activity is decay corrected to 1994.

$\mathrm{N}^{\mathrm{a}}$ stands for number of individual samples. 
Table C-4. Cesium-137 radionuclide concentration summary for all soil profiles taken from the 1978 NMIRS together with our most recent trips in 1986 through 1989 on Rongelap Island (F42).

\begin{tabular}{|c|c|c|c|c|c|c|c|c|}
\hline \multirow{2}{*}{$\begin{array}{l}\text { Soil } \\
\text { depth } \\
(\mathrm{cm})\end{array}$} & \multirow[b]{2}{*}{$\mathrm{Na}^{\mathrm{a}}$} & \multicolumn{5}{|c|}{ pCi $g^{-1}$ dry wt } & \multirow{2}{*}{$\begin{array}{l}\text { Mean } \\
\text { of logs }\end{array}$} & \multirow{2}{*}{$\begin{array}{c}\text { SD } \\
\text { of logs } \\
\end{array}$} \\
\hline & & Minimum & Maximum & Median & Mean & $\mathrm{SD}$ & & \\
\hline $0-05$ & 294 & $8.3 \times 10^{-2}$ & $5.7 \times 10^{1}$ & $1.4 \times 10^{1}$ & $1.4 \times 10^{1}$ & $9.6 \times 10^{0}$ & $2.3 \times 10^{0}$ & $1.1 \times 10^{0}$ \\
\hline $05-10$ & 114 & $6.9 \times 10^{-2}$ & $3.3 \times 10^{1}$ & $6.5 \times 10^{0}$ & $7.4 \times 10^{0}$ & $5.8 \times 10^{0}$ & $1.7 \times 10^{0}$ & $9.3 \times 10^{-1}$ \\
\hline $10-15$ & 114 & $2.0 \times 10^{-1}$ & $3.2 \times 10^{1}$ & $2.9 \times 10^{0}$ & $4.0 \times 10^{0}$ & $4.2 \times 10^{0}$ & $9.5 \times 10^{-1}$ & $9.8 \times 10^{-1}$ \\
\hline $15-25$ & 115 & $3.9 \times 10^{-2}$ & $1.2 \times 10^{1}$ & $1.2 \times 10^{0}$ & $1.8 \times 10^{0}$ & $1.8 \times 10^{0}$ & $7.2 \times 10^{-2}$ & $1.1 \times 10^{0}$ \\
\hline $25-40$ & 113 & $2.4 \times 10^{-2}$ & $3.0 \times 10^{0}$ & $3.6 \times 10^{-1}$ & $6.0 \times 10^{-1}$ & $6.6 \times 10^{-1}$ & $-1.0 \times 10^{0}$ & $1.0 \times 10^{0}$ \\
\hline $40-60$ & 93 & $2.9 \times 10^{-2}$ & $2.4 \times 10^{0}$ & $1.9 \times 10^{-1}$ & $3.6 \times 10^{-1}$ & $4.6 \times 10^{-1}$ & $-1.6 \times 10^{0}$ & $1.1 \times 10^{0}$ \\
\hline $0-05$ & 294 & $8.3 \times 10^{-2}$ & $5.7 \times 10^{1}$ & $1.4 \times 10^{1}$ & $1.4 \times 10^{1}$ & $9.6 \times 10^{0}$ & $2.3 \times 10^{0}$ & $1.1 \times 10^{0}$ \\
\hline $0-10$ & 113 & $8.5 \times 10^{-2}$ & $3.8 \times 10^{1}$ & $9.4 \times 10^{0}$ & $1.1 \times 10^{1}$ & $7.3 \times 10^{0}$ & $2.2 \times 10^{0}$ & $8.7 \times 10^{-1}$ \\
\hline $0-15$ & 111 & $1.2 \times 10^{-1}$ & $3.4 \times 10^{1}$ & $7.5 \times 10^{0}$ & $8.8 \times 10^{0}$ & $5.7 \times 10^{0}$ & $1.9 \times 10^{0}$ & $8.0 \times 10^{-1}$ \\
\hline $0-25$ & 110 & $1.7 \times 10^{-1}$ & $2.5 \times 10^{1}$ & $5.1 \times 10^{0}$ & $6.0 \times 10^{0}$ & $3.8 \times 10^{0}$ & $1.6 \times 10^{0}$ & $7.0 \times 10^{-1}$ \\
\hline $0-40$ & 108 & $1.3 \times 10^{-1}$ & $1.6 \times 10^{1}$ & $3.5 \times 10^{0}$ & $3.9 \times 10^{0}$ & $2.4 \times 10^{0}$ & $1.2 \times 10^{0}$ & $6.7 \times 10^{-1}$ \\
\hline $0-60$ & 88 & $1.4 \times 10^{-1}$ & $1.1 \times 10^{1}$ & $2.4 \times 10^{0}$ & $2.7 \times 10^{0}$ & $1.7 \times 10^{0}$ & $8.0 \times 10^{-1}$ & $6.5 \times 10^{-1}$ \\
\hline
\end{tabular}

NOTE: Specific activity is decay corrected to 1994. $\mathrm{N}^{\mathrm{a}}$ stands for number of individual samples. 
Table C-5. Cesium-137 radionuclide concentration summary for all soil profiles taken from the 1978 NMIRS together with our most recent trip in 1991 on Arbar Island (F43).

\begin{tabular}{|c|c|c|c|c|c|c|c|c|}
\hline \multirow{2}{*}{$\begin{array}{r}\text { Soil } \\
\text { depth } \\
(\mathrm{cm})\end{array}$} & \multirow[b]{2}{*}{$\mathrm{Na}$} & \multicolumn{5}{|c|}{ pCig ${ }^{-1}$ dry wt } & \multirow{2}{*}{$\begin{array}{l}\text { Mean } \\
\text { of logs }\end{array}$} & \multirow{2}{*}{$\begin{array}{c}\mathrm{SD} \\
\text { of logs } \\
\end{array}$} \\
\hline & & Minimum & Maximum & Median & Mean & $\mathrm{SD}$ & & \\
\hline $0-05$ & 13 & $4.0 \times 10^{0}$ & $3.8 \times 10^{1}$ & $1.0 \times 10^{1}$ & $1.4 \times 10^{1}$ & $1.1 \times 10^{1}$ & $2.4 \times 10^{0}$ & $6.7 \times 10^{-1}$ \\
\hline $05-10$ & 13 & $2.0 \times 10^{0}$ & $3.7 \times 10^{1}$ & $7.3 \times 10^{0}$ & $1.1 \times 10^{1}$ & $1.0 \times 10^{1}$ & $2.0 \times 10^{0}$ & $9.5 \times 10^{-1}$ \\
\hline $10-15$ & 13 & $6.9 \times 10^{-1}$ & $2.4 \times 10^{1}$ & $2.4 \times 10^{0}$ & $6.6 \times 10^{0}$ & $8.2 \times 10^{0}$ & $1.2 \times 10^{0}$ & $1.2 \times 10^{0}$ \\
\hline $15-25$ & 12 & $1.5 \times 10^{-1}$ & $1.6 \times 10^{1}$ & $1.5 \times 10^{0}$ & $3.3 \times 10^{0}$ & $4.9 \times 10^{0}$ & $3.8 \times 10^{-1}$ & $1.3 \times 10^{0}$ \\
\hline $25-40$ & 11 & $1.2 \times 10^{-2}$ & $1.8 \times 10^{0}$ & $1.0 \times 10^{0}$ & $8.0 \times 10^{-1}$ & $6.4 \times 10^{-1}$ & $-8.7 \times 10^{-1}$ & $1.6 \times 10^{0}$ \\
\hline $40-60$ & 4 & $5.9 \times 10^{-2}$ & $1.2 \times 10^{0}$ & $3.1 \times 10^{-1}$ & $4.7 \times 10^{-1}$ & $5.3 \times 10^{-1}$ & $-1.5 \times 10^{0}$ & $1.5 \times 10^{0}$ \\
\hline $0-05$ & 13 & $4.0 \times 10^{0}$ & $3.8 \times 10^{1}$ & $1.0 \times 10^{1}$ & $1.4 \times 10^{1}$ & $1.1 \times 10^{1}$ & $2.4 \times 10^{0}$ & $6.7 \times 10^{-1}$ \\
\hline $0-10$ & 12 & $3.0 \times 10^{0}$ & $3.6 \times 10^{1}$ & $1.0 \times 10^{1}$ & $1.3 \times 10^{1}$ & $9.1 \times 10^{0}$ & $2.3 \times 10^{0}$ & $7.1 \times 10^{-1}$ \\
\hline $0-15$ & 12 & $2.5 \times 10^{0}$ & $3.2 \times 10^{1}$ & $7.3 \times 10^{0}$ & $1.1 \times 10^{1}$ & $8.3 \times 10^{0}$ & $2.1 \times 10^{0}$ & $7.5 \times 10^{-1}$ \\
\hline $0-25$ & 11 & $1.7 \times 10^{0}$ & $2.3 \times 10^{1}$ & $5.3 \times 10^{0}$ & $8.0 \times 10^{0}$ & $6.6 \times 10^{0}$ & $1.8 \times 10^{0}$ & $8.0 \times 10^{-1}$ \\
\hline $0-40$ & 9 & $1.1 \times 10^{0}$ & $1.5 \times 10^{1}$ & $3.7 \times 10^{0}$ & $5.5 \times 10^{0}$ & $4.7 \times 10^{0}$ & $1.4 \times 10^{0}$ & $8.8 \times 10^{-1}$ \\
\hline 0.60 & 3 & $7.2 \times 10^{-1}$ & $4.2 \times 10^{0}$ & $2.3 \times 10^{0}$ & $2.4 \times 10^{0}$ & $1.8 \times 10^{\circ}$ & $6.5 \times 10^{-1}$ & $9.0 \times 10^{-1}$ \\
\hline
\end{tabular}

NOTE: Specific activity is decay corrected to 1994 .

$\mathrm{Na}^{\mathrm{a}}$ stands for number of individual samples. 
Table C-6. Cesium-137 radionuclide concentration summary for all soil profiles taken from our most recent trip in 1991 on Eniran Island (F46).

\begin{tabular}{|c|c|c|c|c|c|c|c|c|}
\hline \multirow{2}{*}{$\begin{array}{r}\text { Soil } \\
\text { depth } \\
(\mathrm{cm}) \\
\end{array}$} & \multirow[b]{2}{*}{$\mathrm{Na}^{\mathrm{a}}$} & \multicolumn{5}{|c|}{$\mathrm{pCi} \mathrm{g}^{-1}$ dry wt } & \multirow{2}{*}{$\begin{array}{l}\text { Mean } \\
\text { of logs }\end{array}$} & \multirow{2}{*}{$\begin{array}{c}S D \\
\text { of logs }\end{array}$} \\
\hline & & Minimum & Maximum & Median & Mean & $\mathrm{SD}$ & & \\
\hline $0-05$ & 6 & $6.2 \times 10^{0}$ & $2.2 \times 10^{1}$ & $1.3 \times 10^{1}$ & $1.3 \times 10^{1}$ & $6.0 \times 10^{0}$ & $2.5 \times 10^{0}$ & $4.8 \times 10^{-1}$ \\
\hline $05-10$ & 7 & $1.7 \times 10^{0}$ & $2.3 \times 10^{1}$ & $8.3 \times 10^{0}$ & $9.8 \times 10^{0}$ & $7.3 \times 10^{0}$ & $2.0 \times 10^{0}$ & $8.6 \times 10^{-1}$ \\
\hline $10-15$ & 7 & $5.3 \times 10^{-1}$ & $7.3 \times 10^{0}$ & $3.3 \times 10^{0}$ & $3.9 \times 10^{0}$ & $2.5 \times 10^{0}$ & $1.1 \times 10^{0}$ & $9.1 \times 10^{-1}$ \\
\hline $15-25$ & 7 & $1.4 \times 10^{-1}$ & $6.1 \times 10^{0}$ & $2.0 \times 10^{0}$ & $2.4 \times 10^{0}$ & $2.3 \times 10^{0}$ & $3.4 \times 10^{-1}$ & $1.3 \times 10^{0}$ \\
\hline $25-40$ & 7 & $4.2 \times 10^{-2}$ & $2.5 \times 10^{0}$ & $3.4 \times 10^{-1}$ & $9.1 \times 10^{-1}$ & $1.0 \times 10^{0}$ & $-8.4 \times 10^{-1}$ & $1.5 \times 10^{0}$ \\
\hline $40-60$ & 0 & $0.0 \times 10^{0}$ & $0.0 \times 10^{0}$ & $0.0 \times 10^{0}$ & $0.0 \times 10^{0}$ & $0.0 \times 10^{0}$ & $0.0 \times 10^{0}$ & $0.0 \times 10^{0}$ \\
\hline $0-05$ & 6 & $6.2 \times 10^{0}$ & $2.2 \times 10^{1}$ & $1.3 \times 10^{1}$ & $1.3 \times 10^{1}$ & $6.0 \times 10^{0}$ & $2.5 \times 10^{0}$ & $4.8 \times 10^{-1}$ \\
\hline $0-10$ & 6 & $6.6 \times 10^{0}$ & $1.9 \times 10^{1}$ & $1.2 \times 10^{1}$ & $1.2 \times 10^{1}$ & $5.5 \times 10^{0}$ & $2.4 \times 10^{0}$ & $4.7 \times 10^{-1}$ \\
\hline $0-15$ & 6 & $5.5 \times 10^{0}$ & $1.5 \times 10^{1}$ & $9.2 \times 10^{0}$ & $9.7 \times 10^{0}$ & $3.9 \times 10^{0}$ & $2.2 \times 10^{0}$ & $4.1 \times 10^{-1}$ \\
\hline $0-25$ & 6 & $3.7 \times 10^{0}$ & $9.6 \times 10^{0}$ & $6.6 \times 10^{0}$ & $6.9 \times 10^{0}$ & $2.1 \times 10^{0}$ & $1.9 \times 10^{0}$ & $3.3 \times 10^{-1}$ \\
\hline $0-40$ & 6 & $2.4 \times 10^{0}$ & $6.1 \times 10^{0}$ & $4.7 \times 10^{0}$ & $4.7 \times 10^{0}$ & $1.4 \times 10^{0}$ & $1.5 \times 10^{\circ}$ & $3.4 \times 10^{-1}$ \\
\hline $0-60$ & 0 & $0.0 \times 10^{0}$ & $0.0 \times 10^{0}$ & $0.0 \times 10^{0}$ & $0.0 \times 10^{0}$ & $0.0 \times 10^{0}$ & $0.0 \times 10^{0}$ & $0.0 \times 10^{0}$ \\
\hline
\end{tabular}

NOTE: Specific activity is decay corrected to 1994.

$\mathrm{Na}$ stands for number of individual samples. 
Table C-7. Cesium-137 radionuclide concentration summary for all soil profiles taken from our most recent trip in 1991 on Tufa Island (F47).

\begin{tabular}{|c|c|c|c|c|c|c|c|c|}
\hline \multirow{2}{*}{$\begin{array}{l}\text { Soil } \\
\text { depth } \\
(\mathrm{cm})\end{array}$} & \multirow[b]{2}{*}{$\mathrm{Na}^{\mathrm{a}}$} & \multicolumn{5}{|c|}{ pCi $^{-1}$ dry wt } & \multirow{2}{*}{$\begin{array}{l}\text { Mean } \\
\text { of logs }\end{array}$} & \multirow{2}{*}{$\begin{array}{c}\mathrm{SD} \\
\text { of logs } \\
\end{array}$} \\
\hline & & Minimum & Maximum & Median & Mean & $\mathrm{SD}$ & & \\
\hline $0-05$ & 8 & $6.5 \times 10^{0}$ & $6.1 \times 10^{1}$ & $1.8 \times 10^{1}$ & $2.4 \times 10^{1}$ & $1.8 \times 10^{1}$ & $2.9 \times 10^{0}$ & $7.0 \times 10^{-1}$ \\
\hline $05-10$ & 8 & $6.2 \times 10^{0}$ & $5.5 \times 10^{1}$ & $2.1 \times 10^{1}$ & $2.2 \times 10^{1}$ & $1.6 \times 10^{1}$ & $2.9 \times 10^{0}$ & $7.6 \times 10^{-1}$ \\
\hline $10-15$ & 8 & $2.0 \times 10^{0}$ & $3.1 \times 10^{1}$ & $8.4 \times 10^{0}$ & $1.1 \times 10^{1}$ & $9.5 \times 10^{0}$ & $2.0 \times 10^{0}$ & $9.3 \times 10^{-1}$ \\
\hline $15-25$ & 8 & $1.1 \times 10^{0}$ & $1.6 \times 10^{1}$ & $3.0 \times 10^{0}$ & $4.4 \times 10^{0}$ & $4.8 \times 10^{0}$ & $1.1 \times 10^{0}$ & $8.4 \times 10^{-1}$ \\
\hline $25-40$ & 8 & $2.5 \times 10^{-1}$ & $5.2 \times 10^{0}$ & $1.1 \times 10^{0}$ & $1.9 \times 10^{0}$ & $1.8 \times 10^{0}$ & $1.9 \times 10^{-1}$ & $1.1 \times 10^{0}$ \\
\hline $40-60$ & 0 & $0.0 \times 10^{0}$ & $0.0 \times 10^{0}$ & $0.0 \times 10^{0}$ & $0.0 \times 10^{0}$ & $0.0 \times 10^{0}$ & $0.0 \times 10^{0}$ & $0.0 \times 10^{0}$ \\
\hline $0-05$ & 8 & $6.5 \times 10^{0}$ & $6.1 \times 10^{1}$ & $1.8 \times 10^{1}$ & $2.4 \times 10^{1}$ & $1.8 \times 10^{1}$ & $2.9 \times 10^{0}$ & $7.0 \times 10^{-1}$ \\
\hline $0-10$ & 8 & $1.0 \times 10^{1}$ & $4.5 \times 10^{1}$ & $1.7 \times 10^{1}$ & $2.3 \times 10^{1}$ & $1.4 \times 10^{1}$ & $3.0 \times 10^{0}$ & $6.0 \times 10^{-1}$ \\
\hline $0-15$ & 8 & $7.9 \times 10^{0}$ & $3.6 \times 10^{1}$ & $1.5 \times 10^{1}$ & $1.9 \times 10^{1}$ & $1.1 \times 10^{1}$ & $2.8 \times 10^{0}$ & $6.1 \times 10^{-1}$ \\
\hline $0-25$ & 8 & $5.3 \times 10^{0}$ & $2.8 \times 10^{1}$ & $9.8 \times 10^{0}$ & $1.3 \times 10^{1}$ & $8.1 \times 10^{0}$ & $2.4 \times 10^{0}$ & $6.0 \times 10^{-1}$ \\
\hline $0-40$ & 8 & $3.5 \times 10^{0}$ & $2.0 \times 10^{1}$ & $6.9 \times 10^{0}$ & $8.8 \times 10^{0}$ & $5.6 \times 10^{0}$ & $2.0 \times 10^{0}$ & $6.1 \times 10^{-1}$ \\
\hline $0-60$ & 0 & $0.0 \times 10^{0}$ & $0.0 \times 10^{0}$ & $0.0 \times 10^{0}$ & $0.0 \times 10^{0}$ & $0.0 \times 10^{0}$ & $0.0 \times 10^{0}$ & $0.0 \times 10^{0}$ \\
\hline
\end{tabular}

NOTE: Specific activity is decay corrected to 1994.

$\mathrm{N}^{\mathrm{a}}$ stands for number of individual samples. 
Table C-8. Cesium-137 radionuclide concentration summary for all soil profiles taken from the 1978 NMIRS together with our most recent trip in 1989 on Borukka Island (F49).

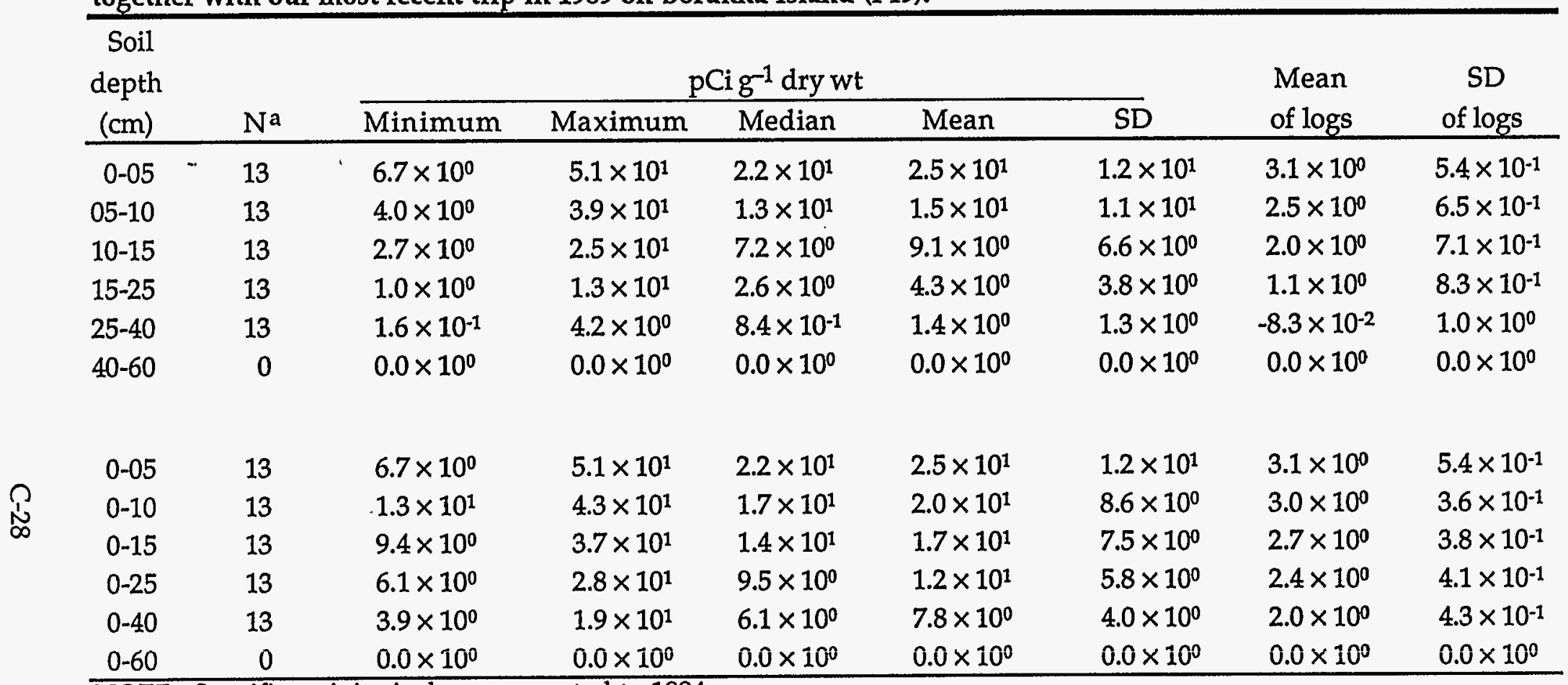

NOTE: Specific activity is decay corrected to 1994.

$\mathrm{N}^{\mathrm{a}}$ stands for number of individual samples. 
Table C-9. Strontium-90 radionuclide concentration summary for all soil profiles taken during the 1978 NMIRS on Eniaetok Island (F33).

\begin{tabular}{|c|c|c|c|c|c|c|c|c|}
\hline \multirow{2}{*}{$\begin{array}{r}\text { Soil } \\
\text { depth } \\
(\mathrm{cm})\end{array}$} & \multirow[b]{2}{*}{$\mathrm{Na}^{\mathrm{a}}$} & \multicolumn{5}{|c|}{$\mathrm{pCi}^{-1}$ dry wt } & \multirow{2}{*}{$\begin{array}{r}\text { Mean } \\
\text { of logs }\end{array}$} & \multirow{2}{*}{$\begin{array}{c}\mathrm{SD} \\
\text { of logs } \\
\end{array}$} \\
\hline & & Minimum & Maximum & Median & Mean & SD & & \\
\hline $0-05$ & 6 & $7.3 \times 10^{-1}$ & $1.5 \times 10^{1}$ & $5.4 \times 10^{0}$ & $6.6 \times 10^{0}$ & $5.5 \times 10^{0}$ & $1.5 \times 10^{0}$ & $1.2 \times 10^{0}$ \\
\hline $05-10$ & 6 & $2.9 \times 10^{0}$ & $2.0 \times 10^{1}$ & $8.1 \times 10^{0}$ & $9.2 \times 10^{0}$ & $6.0 \times 10^{0}$ & $2.0 \times 10^{0}$ & $6.9 \times 10^{-1}$ \\
\hline $10-15$ & 6 & $1.5 \times 10^{0}$ & $1.4 \times 10^{1}$ & $3.4 \times 10^{0}$ & $6.1 \times 10^{0}$ & $5.3 \times 10^{0}$ & $1.5 \times 10^{0}$ & $8.8 \times 10^{-1}$ \\
\hline $15-25$ & 6 & $5.3 \times 10^{-1}$ & $7.9 \times 10^{0}$ & $2.1 \times 10^{0}$ & $3.3 \times 10^{0}$ & $2.9 \times 10^{0}$ & $8.6 \times 10^{-1}$ & $9.7 \times 10^{-1}$ \\
\hline $25-40$ & 6 & $3.2 \times 10^{-1}$ & $2.3 \times 10^{1}$ & $3.1 \times 10^{0}$ & $5.9 \times 10^{0}$ & $8.4 \times 10^{0}$ & $1.0 \times 10^{0}$ & $1.4 \times 10^{0}$ \\
\hline $40-60$ & 6 & $2.4 \times 10^{-1}$ & $4.9 \times 10^{0}$ & $2.7 \times 10^{0}$ & $2.4 \times 10^{0}$ & $1.8 \times 10^{0}$ & $4.9 \times 10^{-1}$ & $1.2 \times 10^{0}$ \\
\hline $0-05$ & 6 & $7.3 \times 10^{-1}$ & $1.5 \times 10^{1}$ & $5.4 \times 10^{0}$ & $6.6 \times 10^{0}$ & $5.5 \times 10^{0}$ & $1.5 \times 10^{0}$ & $1.2 \times 10^{0}$ \\
\hline $0-10$ & 6 & $1.8 \times 10^{0}$ & $1.7 \times 10^{1}$ & $6.8 \times 10^{0}$ & $7.9 \times 10^{0}$ & $5.7 \times 10^{0}$ & $1.8 \times 10^{0}$ & $8.3 \times 10^{-1}$ \\
\hline $0-15$ & 6 & $1.7 \times 10^{0}$ & $1.6 \times 10^{1}$ & $5.9 \times 10^{0}$ & $7.3 \times 10^{0}$ & $4.8 \times 10^{0}$ & $1.8 \times 10^{0}$ & $7.3 \times 10^{-1}$ \\
\hline $0-25$ & 6 & $1.3 \times 10^{0}$ & $1.2 \times 10^{1}$ & $4.4 \times 10^{0}$ & $5.7 \times 10^{0}$ & $3.8 \times 10^{0}$ & $1.5 \times 10^{0}$ & $7.8 \times 10^{-1}$ \\
\hline $0-40$ & 6 & $9.0 \times 10^{-1}$ & $1.4 \times 10^{1}$ & $4.0 \times 10^{0}$ & $5.8 \times 10^{0}$ & $4.7 \times 10^{0}$ & $1.4 \times 10^{0}$ & $9.4 \times 10^{-1}$ \\
\hline $0-60$ & 6 & $6.8 \times 10^{-1}$ & $1.0 \times 10^{1}$ & $4.0 \times 10^{0}$ & $4.7 \times 10^{0}$ & $3.4 \times 10^{0}$ & $1.2 \times 10^{0}$ & $9.5 \times 10^{-1}$ \\
\hline
\end{tabular}

NOTE: Specific activity is decay corrected to 1994.

$\mathrm{Na}^{\mathrm{a}}$ stands for number of individual samples. 
Table C-10. Strontium-90 radionuclide concentration summary for all soil profiles taken during the 1978 NMIRS on Rongelap Island (F42).

\begin{tabular}{|c|c|c|c|c|c|c|c|c|}
\hline \multirow{2}{*}{$\begin{array}{r}\text { Soil } \\
\text { depth } \\
(\mathrm{cm})\end{array}$} & \multirow[b]{2}{*}{$\mathrm{Na}$} & \multicolumn{5}{|c|}{ pCig $^{-1}$ dry wt } & \multirow{2}{*}{$\begin{array}{l}\text { Mean } \\
\text { of logs } \\
\end{array}$} & \multirow{2}{*}{$\begin{array}{c}S D \\
\text { of logs } \\
\end{array}$} \\
\hline & & Minimum & Maximum & Median & Mean & $\mathrm{SD}$ & & \\
\hline $0-05$ & 20 & $1.2 \times 10^{-1}$ & $1.3 \times 10^{1}$ & $5.2 \times 10^{0}$ & $4.8 \times 10^{0}$ & $3.1 \times 10^{0}$ & $1.2 \times 10^{0}$ & $1.1 \times 10^{0}$ \\
\hline $05-10$ & 20 & $1.6 \times 10^{-1}$ & $1.6 \times 10^{1}$ & $3.3 \times 10^{0}$ & $5.5 \times 10^{0}$ & $5.0 \times 10^{0}$ & $1.2 \times 10^{0}$ & $1.2 \times 10^{0}$ \\
\hline $10-15$ & 20 & $2.4 \times 10^{-2}$ & $1.4 \times 10^{1}$ & $3.1 \times 10^{0}$ & $4.1 \times 10^{0}$ & $4.1 \times 10^{0}$ & $7.6 \times 10^{-1}$ & $1.5 \times 10^{0}$ \\
\hline $15-25$ & 20 & $3.9 \times 10^{-1}$ & $8.6 \times 10^{0}$ & $2.2 \times 10^{0}$ & $3.1 \times 10^{0}$ & $2.4 \times 10^{0}$ & $7.7 \times 10^{-1}$ & $9.3 \times 10^{-1}$ \\
\hline $25-40$ & 21 & $1.1 \times 10^{-2}$ & $7.5 \times 10^{0}$ & $1.4 \times 10^{0}$ & $1.8 \times 10^{0}$ & $1.7 \times 10^{0}$ & $-3.8 \times 10^{-2}$ & $1.5 \times 10^{0}$ \\
\hline $40-60$ & 0 & $0.0 \times 10^{0}$ & $0.0 \times 10^{0}$ & $0.0 \times 10^{0}$ & $0.0 \times 10^{0}$ & $0.0 \times 10^{0}$ & $0.0 \times 10^{0}$ & $0.0 \times 10^{0}$ \\
\hline $0-05$ & 20 & $1.2 \times 10^{-1}$ & $1.3 \times 10^{1}$ & $5.2 \times 10^{0}$ & $4.8 \times 10^{0}$ & $3.1 \times 10^{0}$ & $1.2 \times 10^{0}$ & $1.1 \times 10^{0}$ \\
\hline $0-10$ & 19 & $1.4 \times 10^{-1}$ & $1.5 \times 10^{1}$ & $4.1 \times 10^{0}$ & $5.2 \times 10^{0}$ & $3.9 \times 10^{0}$ & $1.3 \times 10^{0}$ & $1.1 \times 10^{0}$ \\
\hline $0-15$ & 18 & $2.8 \times 10^{-1}$ & $1.3 \times 10^{1}$ & $3.7 \times 10^{0}$ & $4.8 \times 10^{0}$ & $3.8 \times 10^{0}$ & $1.2 \times 10^{0}$ & $1.0 \times 10^{0}$ \\
\hline $0-25$ & 17 & $7.6 \times 10^{-1}$ & $9.9 \times 10^{0}$ & $3.6 \times 10^{0}$ & $4.2 \times 10^{0}$ & $3.1 \times 10^{0}$ & $1.1 \times 10^{0}$ & $8.5 \times 10^{-1}$ \\
\hline $0-40$ & 17 & $6.1 \times 10^{-1}$ & $7.4 \times 10^{0}$ & $2.9 \times 10^{0}$ & $3.3 \times 10^{0}$ & $2.2 \times 10^{0}$ & $9.2 \times 10^{-1}$ & $8.3 \times 10^{-1}$ \\
\hline $0-60$ & 0 & $0.0 \times 10^{0}$ & $0.0 \times 10^{0}$ & $0.0 \times 10^{0}$ & $0.0 \times 10^{0}$ & $0.0 \times 10^{0}$ & $0.0 \times 10^{0}$ & $0.0 \times 10^{0}$ \\
\hline
\end{tabular}

NOTE: Specific activity is decay corrected to 1994.

$\mathrm{N}^{\mathrm{a}}$ stands for number of individual samples. 
Table C-11. Strontium-90 radionuclide concentration summary for all soil profiles taken during the 1978 NMIRS on Borukka Island (F49).

\begin{tabular}{|c|c|c|c|c|c|c|c|c|}
\hline \multirow{2}{*}{$\begin{array}{r}\text { Soil } \\
\text { depth } \\
(\mathrm{cm})\end{array}$} & \multirow[b]{2}{*}{$\mathrm{Na}^{\mathrm{a}}$} & \multicolumn{5}{|c|}{$\mathrm{pCig}^{-1}$ dry wt } & \multirow{2}{*}{$\begin{array}{l}\text { Mean } \\
\text { of logs } \\
\end{array}$} & \multirow{2}{*}{$\begin{array}{c}S D \\
\text { of logs } \\
\end{array}$} \\
\hline & & Minimum & Maximum & Median & Mean & $\mathrm{SD}$ & & \\
\hline $0-05$ & 3 & $1.1 \times 10^{1}$ & $1.2 \times 10^{1}$ & $1.2 \times 10^{1}$ & $1.2 \times 10^{1}$ & $6.1 \times 10^{-1}$ & $2.5 \times 10^{0}$ & $5.4 \times 10^{-2}$ \\
\hline $05-10$ & 3 & $3.8 \times 10^{0}$ & $9.9 \times 10^{0}$ & $9.3 \times 10^{0}$ & $7.6 \times 10^{0}$ & $3.4 \times 10^{0}$ & $2.0 \times 10^{0}$ & $5.4 \times 10^{-1}$ \\
\hline $10-15$ & 3 & $3.4 \times 10^{0}$ & $7.3 \times 10^{0}$ & $6.2 \times 10^{0}$ & $5.6 \times 10^{0}$ & $2.0 \times 10^{0}$ & $1.7 \times 10^{0}$ & $4.0 \times 10^{-1}$ \\
\hline $15-25$ & 3 & $1.6 \times 10^{0}$ & $5.2 \times 10^{0}$ & $2.4 \times 10^{0}$ & $3.0 \times 10^{0}$ & $1.9 \times 10^{0}$ & $9.8 \times 10^{-1}$ & $6.1 \times 10^{-1}$ \\
\hline $25-40$ & 2 & $1.7 \times 10^{0}$ & $3.2 \times 10^{0}$ & $2.5 \times 10^{0}$ & $2.5 \times 10^{0}$ & $1.1 \times 10^{0}$ & $8.5 \times 10^{-1}$ & $4.5 \times 10^{-1}$ \\
\hline $40-60$ & 0 & $0.0 \times 10^{0}$ & $0.0 \times 10^{0}$ & $0.0 \times 10^{0}$ & $0.0 \times 10^{0}$ & $0.0 \times 10^{0}$ & $0.0 \times 10^{0}$ & $0.0 \times 10^{0}$ \\
\hline $0-05$ & 3 & $1.1 \times 10^{1}$ & $1.2 \times 10^{1}$ & $1.2 \times 10^{1}$ & $1.2 \times 10^{1}$ & $6.1 \times 10^{-1}$ & $2.5 \times 10^{0}$ & $5.4 \times 10^{-2}$ \\
\hline $0-10$ & 3 & $7.8 \times 10^{0}$ & $1.1 \times 10^{1}$ & $1.0 \times 10^{1}$ & $9.6 \times 10^{0}$ & $1.5 \times 10^{0}$ & $2.3 \times 10^{0}$ & $1.7 \times 10^{-1}$ \\
\hline $0-15$ & 3 & $6.4 \times 10^{0}$ & $9.5 \times 10^{0}$ & $9.0 \times 10^{0}$ & $8.3 \times 10^{0}$ & $1.7 \times 10^{0}$ & $2.1 \times 10^{0}$ & $2.2 \times 10^{-1}$ \\
\hline $0-25$ & 3 & $4.8 \times 10^{0}$ & $7.8 \times 10^{0}$ & $6.0 \times 10^{0}$ & $6.2 \times 10^{0}$ & $1.5 \times 10^{0}$ & $1.8 \times 10^{0}$ & $2.5 \times 10^{-1}$ \\
\hline $0-40$ & 2 & $4.4 \times 10^{0}$ & $6.1 \times 10^{0}$ & $5.2 \times 10^{0}$ & $5.2 \times 10^{0}$ & $1.2 \times 10^{0}$ & $1.6 \times 10^{0}$ & $2.3 \times 10^{-1}$ \\
\hline $0-60$ & 0 & $0.0 \times 10^{0}$ & $0.0 \times 10^{0}$ & $0.0 \times 10^{0}$ & $0.0 \times 10^{0}$ & $0.0 \times 10^{0}$ & $0.0 \times 10^{0}$ & $0.0 \times 10^{0}$ \\
\hline
\end{tabular}

NOTE: Specific activity is decay corrected to 1994.

$N^{a}$ stands for number of individual samples. 
Table C-12. Plutonium-239+240 radionuclide concentration summary for all soil profiles taken during the 1978 NMIRS together with our recent trip in 1989 on Eniaetok Island (F33).

\begin{tabular}{|c|c|c|c|c|c|c|c|c|}
\hline \multirow{2}{*}{$\begin{array}{r}\text { Soil } \\
\text { depth } \\
(\mathrm{cm})\end{array}$} & \multirow[b]{2}{*}{$\mathrm{Na}$} & \multicolumn{5}{|c|}{$\mathrm{pCi} \mathrm{g}^{-1}$ dry wt } & \multirow{2}{*}{$\begin{array}{l}\text { Mean } \\
\text { of logs }\end{array}$} & \multirow{2}{*}{$\begin{array}{c}S D \\
\text { of logs }\end{array}$} \\
\hline & & Minimum & Maximum & Median & Mean & SD & & \\
\hline $0-05$ & 21 & $2.3 \times 10^{-2}$ & $2.0 \times 10^{1}$ & $6.6 \times 10^{0}$ & $7.2 \times 10^{0}$ & $5.9 \times 10^{0}$ & $1.3 \times 10^{0}$ & $1.7 \times 10^{0}$ \\
\hline $05-10$ & 6 & $1.5 \times 10^{0}$ & $7.2 \times 10^{0}$ & $3.0 \times 10^{0}$ & $3.3 \times 10^{0}$ & $2.0 \times 10^{0}$ & $1.1 \times 10^{0}$ & $5.5 \times 10^{-1}$ \\
\hline $10-15$ & 6 & $2.3 \times 10^{-1}$ & $6.9 \times 10^{0}$ & $2.0 \times 10^{0}$ & $2.6 \times 10^{0}$ & $2.6 \times 10^{0}$ & $3.1 \times 10^{-1}$ & $1.4 \times 10^{0}$ \\
\hline $15-25$ & 6 & $4.5 \times 10-03$ & $9.3 \times 10^{0}$ & $3.1 \times 10^{-1}$ & $1.8 \times 10^{0}$ & $3.7 \times 10^{0}$ & $-1.3 \times 10^{0}$ & $2.4 \times 10^{0}$ \\
\hline $25-40$ & 6 & $9.9 \times 10-03$ & $8.7 \times 10^{-2}$ & $5.9 \times 10^{-2}$ & $5.6 \times 10^{-2}$ & $2.6 \times 10^{-2}$ & $-3.1 \times 10^{0}$ & $7.8 \times 10^{-1}$ \\
\hline $40-60$ & 6 & $1.6 \times 10^{-2}$ & $6.8 \times 10^{-1}$ & $8.9 \times 10^{-2}$ & $1.7 \times 10^{-1}$ & $2.5 \times 10^{-1}$ & $-2.5 \times 10^{0}$ & $1.3 \times 10^{0}$ \\
\hline $0-05$ & 21 & $2.3 \times 10^{-2}$ & $2.0 \times 10^{1}$ & $6.6 \times 10^{0}$ & $7.2 \times 10^{0}$ & $5.9 \times 10^{0}$ & $1.3 \times 10^{0}$ & $1.7 \times 10^{0}$ \\
\hline $0-10$ & 6 & $8.7 \times 10^{-1}$ & $5.9 \times 10^{0}$ & $2.6 \times 10^{0}$ & $2.6 \times 10^{0}$ & $1.8 \times 10^{0}$ & $7.7 \times 10^{-1}$ & $7.1 \times 10^{-1}$ \\
\hline $0-15$ & 6 & $6.5 \times 10^{-1}$ & $6.2 \times 10^{0}$ & $2.1 \times 10^{0}$ & $2.6 \times 10^{0}$ & $1.9 \times 10^{0}$ & $7.5 \times 10^{-1}$ & $7.3 \times 10^{-1}$ \\
\hline $0-25$ & 6 & $3.9 \times 10^{-1}$ & $7.5 \times 10^{0}$ & $1.4 \times 10^{0}$ & $2.3 \times 10^{0}$ & $2.6 \times 10^{0}$ & $4.2 \times 10^{-1}$ & $9.5 \times 10^{-1}$ \\
\hline $0-40$ & 6 & $2.5 \times 10^{-1}$ & $4.7 \times 10^{0}$ & $8.7 \times 10^{-1}$ & $1.4 \times 10^{0}$ & $1.6 \times 10^{0}$ & $-3.3 \times 10^{-2}$ & $9.4 \times 10^{-1}$ \\
\hline $0-60$ & 6 & $1.7 \times 10^{-1}$ & $3.2 \times 10^{0}$ & $7.1 \times 10^{-1}$ & $1.0 \times 10^{0}$ & $1.1 \times 10^{0}$ & $-3.6 \times 10^{-1}$ & $9.4 \times 10^{-1}$ \\
\hline
\end{tabular}

NOTE: Specific activity is decay corrected to 1994.

$\mathrm{N}^{\mathrm{a}}$ stands for number of individual samples. 
Table C-13. Plutonium-239+240 radionuclide concentration summary for all soil profiles taken during the 1978 NMIRS together with our recent trip in 1986 on Rongelap Island (F42).

\begin{tabular}{|c|c|c|c|c|c|c|c|c|}
\hline \multirow{2}{*}{$\begin{array}{r}\text { Soil } \\
\text { depth } \\
(\mathrm{cm})\end{array}$} & \multirow[b]{2}{*}{$\mathrm{Na}^{\mathrm{a}}$} & \multicolumn{5}{|c|}{ pCi $g^{-1}$ dry wt } & \multirow{2}{*}{$\begin{array}{l}\text { Mean } \\
\text { of logs } \\
\end{array}$} & \multirow{2}{*}{$\begin{array}{c}\text { SD } \\
\text { of logs } \\
\end{array}$} \\
\hline & & Minimum & Maximum & Median & Mean & SD & & \\
\hline $0-05$ & 77 & $2.7 \times 10^{-1}$ & $1.3 \times 10^{1}$ & $2.8 \times 10^{0}$ & $3.4 \times 10^{0}$ & $2.8 \times 10^{0}$ & $8.8 \times 10^{-1}$ & $9.3 \times 10^{-1}$ \\
\hline $05-10$ & 16 & $2.1 \times 10^{-1}$ & $1.0 \times 10^{1}$ & $1.0 \times 10^{0}$ & $2.1 \times 10^{0}$ & $2.5 \times 10^{0}$ & $2.0 \times 10^{-1}$ & $1.1 \times 10^{0}$ \\
\hline $10-15$ & 18 & $4.7 \times 10^{-2}$ & $3.5 \times 10^{0}$ & $5.0 \times 10^{-1}$ & $9.2 \times 10^{-1}$ & $9.8 \times 10^{-1}$ & $-7.0 \times 10^{-1}$ & $1.2 \times 10^{0}$ \\
\hline $15-25$ & 18 & $1.8 \times 10^{-2}$ & $1.1 \times 10^{0}$ & $2.0 \times 10^{-1}$ & $2.7 \times 10^{-1}$ & $2.6 \times 10^{-1}$ & $-1.8 \times 10^{0}$ & $1.1 \times 10^{0}$ \\
\hline $5-40$ & 19 & $5.8 \times 10-04$ & $4.9 \times 10^{-1}$ & $9.0 \times 10^{-2}$ & $1.3 \times 10^{-1}$ & $1.3 \times 10^{-1}$ & $-2.8 \times 10^{0}$ & $1.7 \times 10^{0}$ \\
\hline $40-60$ & 0 & $0.0 \times 10^{0}$ & $0.0 \times 10^{0}$ & $0.0 \times 10^{0}$ & $0.0 \times 10^{0}$ & $0.0 \times 10^{0}$ & $0.0 \times 10^{0}$ & $0.0 \times 10^{0}$ \\
\hline $0-05$ & 77 & $2.7 \times 10^{-1}$ & $1.3 \times 10^{1}$ & $2.8 \times 10^{0}$ & $3.4 \times 10^{0}$ & $2.8 \times 10^{0}$ & $8.8 \times 10^{-1}$ & $9.3 \times 10^{-1}$ \\
\hline $0-10$ & 15 & $3.6 \times 10^{-1}$ & $6.2 \times 10^{0}$ & $1.8 \times 10^{0}$ & $2.6 \times 10^{0}$ & $2.1 \times 10^{0}$ & $5.4 \times 10^{-1}$ & $1.0 \times 10^{0}$ \\
\hline $0-15$ & 14 & $2.7 \times 10^{-1}$ & $5.2 \times 10^{0}$ & $1.4 \times 10^{0}$ & $2.0 \times 10^{0}$ & $1.6 \times 10^{0}$ & $3.0 \times 10^{-1}$ & $1.0 \times 10^{0}$ \\
\hline $0-25$ & 13 & $1.8 \times 10^{-1}$ & $3.5 \times 10^{0}$ & $1.2 \times 10^{0}$ & $1.4 \times 10^{0}$ & $1.1 \times 10^{0}$ & $-7.9 \times 10^{-2}$ & $1.0 \times 10^{0}$ \\
\hline $0-40$ & 13 & $1.1 \times 10^{-1}$ & $2.3 \times 10^{0}$ & $8.2 \times 10^{-1}$ & $9.0 \times 10^{-1}$ & $6.6 \times 10^{-1}$ & $-4.3 \times 10^{-1}$ & $9.4 \times 10^{-1}$ \\
\hline $0-60$ & 0 & $0.0 \times 10^{0}$ & $0.0 \times 10^{0}$ & $0.0 \times 10^{0}$ & $0.0 \times 10^{0}$ & $0.0 \times 10^{0}$ & $0.0 \times 10^{0}$ & $0.0 \times 10^{0}$ \\
\hline
\end{tabular}

NOTE: Specific activity is decay corrected to 1994.

$\mathrm{N}^{\mathrm{a}}$ stands for number of individual samples. 
Table C-14. Plutonium-239+240 radionuclide concentration summary for all soil profiles taken during the 1978 NMIRS together with our recent trip in 1989 on Borukka Island (F49).

\begin{tabular}{|c|c|c|c|c|c|c|c|c|}
\hline \multirow{2}{*}{$\begin{array}{l}\text { Soil } \\
\text { depth } \\
(\mathrm{cm})\end{array}$} & \multirow[b]{2}{*}{$\mathrm{Na}^{\mathrm{a}}$} & \multicolumn{5}{|c|}{ pCi g-1 dry wt } & \multirow{2}{*}{$\begin{array}{l}\text { Mean } \\
\text { of logs }\end{array}$} & \multirow{2}{*}{$\begin{array}{c}\text { SD } \\
\text { of logs } \\
\end{array}$} \\
\hline & & Minimum & Maximum & Median & Mean & $\mathrm{SD}$ & & \\
\hline $0-05$ & 13 & $7.4 \times 10^{-1}$ & $1.6 \times 10^{1}$ & $5.3 \times 10^{0}$ & $6.9 \times 10^{0}$ & $4.0 \times 10^{0}$ & $1.7 \times 10^{0}$ & $7.4 \times 10-1$ \\
\hline $05-10$ & 3 & $5.0 \times 10^{-1}$ & $1.6 \times 10^{0}$ & $6.6 \times 10^{-1}$ & $9.3 \times 10^{-1}$ & $6.1 \times 10^{-1}$ & $-2.1 \times 10^{-1}$ & $6.2 \times 10^{-1}$ \\
\hline $10-15$ & 3 & $2.4 \times 10^{-1}$ & $3.9 \times 10^{-1}$ & $2.5 \times 10^{-1}$ & $2.9 \times 10^{-1}$ & $8.8 \times 10^{-2}$ & $-1.3 \times 10^{0}$ & $2.8 \times 10^{-1}$ \\
\hline $15-25$ & 3 & $9.2 \times 10^{-2}$ & $1.9 \times 10^{-1}$ & $1.8 \times 10^{-1}$ & $1.6 \times 10^{-1}$ & $5.6 \times 10^{-2}$ & $-1.9 \times 10^{0}$ & $4.2 \times 10^{-1}$ \\
\hline $25-40$ & 2 & $4.8 \times 10^{-2}$ & $9.1 \times 10^{-2}$ & $6.9 \times 10^{-2}$ & $6.9 \times 10^{-2}$ & $3.0 \times 10^{-2}$ & $-2.7 \times 10^{0}$ & $4.5 \times 10^{-1}$ \\
\hline $40-60$ & 0 & $0.0 \times 10^{0}$ & $0.0 \times 10^{0}$ & $0.0 \times 10^{0}$ & $0.0 \times 10^{0}$ & $0.0 \times 10^{0}$ & $0.0 \times 10^{0}$ & $0.0 \times 10^{0}$ \\
\hline $0-05$ & 13 & $7.4 \times 10^{-1}$ & $1.6 \times 10^{1}$ & $5.3 \times 10^{0}$ & $6.9 \times 10^{0}$ & $4.0 \times 10^{0}$ & $1.7 \times 10^{0}$ & $7.4 \times 10^{-1}$ \\
\hline $0-10$ & 3 & $2.9 \times 10^{0}$ & $5.0 \times 10^{0}$ & $4.3 \times 10^{0}$ & $4.1 \times 10^{0}$ & $1.1 \times 10^{0}$ & $1.4 \times 10^{0}$ & $2.8 \times 10^{-1}$ \\
\hline $0-15$ & 3 & $2.1 \times 10^{0}$ & $3.4 \times 10^{0}$ & $2.9 \times 10^{0}$ & $2.8 \times 10^{0}$ & $6.8 \times 10^{-1}$ & $1.0 \times 10^{0}$ & $2.6 \times 10^{-1}$ \\
\hline $0-25$ & 3 & $1.3 \times 10^{0}$ & $2.1 \times 10^{0}$ & $1.8 \times 10^{0}$ & $1.7 \times 10^{0}$ & $3.9 \times 10^{-1}$ & $5.4 \times 10^{-1}$ & $2.4 \times 10^{-1}$ \\
\hline $0-40$ & 2 & $1.2 \times 10^{0}$ & $1.3 \times 10^{0}$ & $1.3 \times 10^{0}$ & $1.3 \times 10^{0}$ & $1.2 \times 10^{-1}$ & $2.2 \times 10^{-1}$ & $9.8 \times 10^{-2}$ \\
\hline $0-60$ & 0 & $0.0 \times 10^{\circ}$ & $0.0 \times 10^{0}$ & $0.0 \times 10^{0}$ & $0.0 \times 10^{0}$ & $0.0 \times 10^{0}$ & $0.0 \times 10^{0}$ & $0.0 \times 10^{\circ}$ \\
\hline
\end{tabular}

NOTE: Specific activity is decay corrected to 1994.

$\mathrm{N}^{\mathrm{a}}$ stands for number of individual samples. 
Table C-15. Americium-241 radionuclide concentration summary for all soil profiles taken from our most recent trip in 1991 on Erapuotsu Island (F32).

\begin{tabular}{|c|c|c|c|c|c|c|c|c|}
\hline \multirow{2}{*}{$\begin{array}{r}\text { Soil } \\
\text { depth } \\
(\mathrm{cm})\end{array}$} & \multirow[b]{2}{*}{$\mathrm{N}^{2}$} & \multicolumn{5}{|c|}{ pCi $g^{-1}$ dry wt } & \multirow{2}{*}{$\begin{array}{l}\text { Mean } \\
\text { of logs }\end{array}$} & \multirow{2}{*}{$\begin{array}{c}\text { SD } \\
\text { of logs } \\
\end{array}$} \\
\hline & & Minimum & Maximum & Median & Mean & $\mathrm{SD}$ & & \\
\hline $0-05$ & 3 & $3.2 \times 10^{0}$ & $1.5 \times 10^{1}$ & $5.2 \times 10^{0}$ & $7.7 \times 10^{0}$ & $6.1 \times 10^{0}$ & $1.8 \times 10^{0}$ & $7.8 \times 10^{-1}$ \\
\hline $05-10$ & 2 & $4.5 \times 10^{0}$ & $4.6 \times 10^{0}$ & $4.5 \times 10^{0}$ & $4.5 \times 10^{0}$ & $1.1 \times 10^{-1}$ & $1.5 \times 10^{0}$ & $2.3 \times 10^{-2}$ \\
\hline $10-15$ & 0 & $0.0 \times 10^{0}$ & $0.0 \times 10^{0}$ & $0.0 \times 10^{0}$ & $0.0 \times 10^{0}$ & $0.0 \times 10^{0}$ & $0.0 \times 10^{0}$ & $0.0 \times 10^{0}$ \\
\hline $15-25$ & 0 & $0.0 \times 10^{0}$ & $0.0 \times 10^{0}$ & $0.0 \times 10^{0}$ & $0.0 \times 10^{0}$ & $0.0 \times 10^{0}$ & $0.0 \times 10^{0}$ & $0.0 \times 10^{0}$ \\
\hline $25-40$ & 0 & $0.0 \times 10^{0}$ & $0.0 \times 10^{0}$ & $0.0 \times 10^{0}$ & $0.0 \times 10^{0}$ & $0.0 \times 10^{0}$ & $0.0 \times 10^{0}$ & $0.0 \times 10^{0}$ \\
\hline $40-60$ & 0 & $0.0 \times 10^{0}$ & $0.0 \times 10^{0}$ & $0.0 \times 10^{0}$ & $0.0 \times 10^{0}$ & $0.0 \times 10^{0}$ & $0.0 \times 10^{0}$ & $0.0 \times 10^{0}$ \\
\hline $0-05$ & 3 & $3.2 \times 10^{0}$ & $1.5 \times 10^{1}$ & $5.2 \times 10^{0}$ & $7.7 \times 10^{0}$ & $6.1 \times 10^{0}$ & $1.8 \times 10^{0}$ & $7.8 \times 10^{-1}$ \\
\hline $0-10$ & 2 & $3.9 \times 10^{0}$ & $4.8 \times 10^{0}$ & $4.4 \times 10^{0}$ & $4.4 \times 10^{0}$ & $6.6 \times 10^{-1}$ & $1.5 \times 10^{0}$ & $1.5 \times 10^{-1}$ \\
\hline $0-15$ & 0 & $0.0 \times 10^{0}$ & $0.0 \times 10^{0}$ & $0.0 \times 10^{0}$ & $0.0 \times 10^{0}$ & $0.0 \times 10^{0}$ & $0.0 \times 10^{0}$ & $0.0 \times 10^{0}$ \\
\hline $0-25$ & 0 & $0.0 \times 10^{0}$ & $0.0 \times 10^{0}$ & $0.0 \times 10^{0}$ & $0.0 \times 10^{0}$ & $0.0 \times 10^{0}$ & $0.0 \times 10^{0}$ & $0.0 \times 10^{0}$ \\
\hline $0-40$ & 0 & $0.0 \times 10^{0}$ & $0.0 \times 10^{0}$ & $0.0 \times 10^{0}$ & $0.0 \times 10^{0}$ & $0.0 \times 10^{0}$ & $0.0 \times 10^{0}$ & $0.0 \times 10^{0}$ \\
\hline $0-60$ & 0 & $0.0 \times 10^{0}$ & $0.0 \times 10^{0}$ & $0.0 \times 10^{0}$ & $0.0 \times 10^{0}$ & $0.0 \times 10^{0}$ & $0.0 \times 10^{0}$ & $0.0 \times 10^{0}$ \\
\hline
\end{tabular}

NOTE: Specific activity is decay corrected to 1994 .

$\mathrm{Na}$ stands for number of individual samples. 
Table C-16. Americium-241 radionuclide concentration summary for all soil profiles taken from the 1978 NMIRS together with our most recent trip in 1989 on Eniaetok Island (F33).

\begin{tabular}{|c|c|c|c|c|c|c|c|c|}
\hline \multirow{2}{*}{$\begin{array}{r}\text { Soil } \\
\text { depth } \\
(\mathrm{cm}) \\
\end{array}$} & \multirow[b]{2}{*}{$\mathrm{Na}^{\mathrm{a}}$} & \multicolumn{5}{|c|}{ pCig $^{-1}$ dry wt } & \multirow{2}{*}{$\begin{array}{r}\text { Mean } \\
\text { of logs }\end{array}$} & \multirow{2}{*}{$\begin{array}{r}\text { SD } \\
\text { of logs } \\
\end{array}$} \\
\hline & & Minimum & Maximum & Median & Mean & $\mathrm{SD}$ & & \\
\hline $0-05$ & 18 & $2.4 \times 10^{-1}$ & $1.1 \times 10^{1}$ & $5.5 \times 10^{0}$ & $5.1 \times 10^{0}$ & $3.6 \times 10^{0}$ & $1.2 \times 10^{0}$ & $1.2 \times 10^{0}$ \\
\hline $05-10$ & 15 & $1.9 \times 10^{-1}$ & $8.2 \times 10^{0}$ & $8.8 \times 10^{-1}$ & $1.7 \times 10^{0}$ & $2.1 \times 10^{0}$ & $-3.9 \times 10^{-2}$ & $1.1 \times 10^{0}$ \\
\hline $10-15$ & 9 & $1.1 \times 10^{-1}$ & $2.0 \times 10^{0}$ & $6.1 \times 10^{-1}$ & $7.0 \times 10^{-1}$ & $6.2 \times 10^{-1}$ & $-7.7 \times 10^{-1}$ & $1.0 \times 10^{0}$ \\
\hline $15-25$ & 6 & $5.8 \times 10^{-2}$ & $4.7 \times 10^{-1}$ & $1.1 \times 10^{-1}$ & $1.6 \times 10^{-1}$ & $1.6 \times 10^{-1}$ & $-2.1 \times 10^{0}$ & $7.3 \times 10^{-1}$ \\
\hline $25-40$ & 6 & $1.5 \times 10^{-2}$ & $1.1 \times 10^{-1}$ & $2.7 \times 10^{-2}$ & $4.1 \times 10^{-2}$ & $3.5 \times 10^{-2}$ & $-3.4 \times 10^{0}$ & $6.7 \times 10^{-1}$ \\
\hline $40-60$ & 5 & $2.8 \times 10^{-3}$ & $3.4 \times 10^{-1}$ & $6.7 \times 10^{-2}$ & $1.1 \times 10^{-1}$ & $1.3 \times 10^{-1}$ & $-3.1 \times 10^{0}$ & $1.8 \times 10^{0}$ \\
\hline $0-05$ & 18 & $2.4 \times 10^{-1}$ & $1.1 \times 10^{1}$ & $5.5 \times 10^{0}$ & $5.1 \times 10^{0}$ & $3.6 \times 10^{0}$ & $1.2 \times 10^{0}$ & $1.2 \times 10^{0}$ \\
\hline $0-10$ & 15 & $3.9 \times 10^{-1}$ & $6.9 \times 10^{0}$ & $2.8 \times 10^{0}$ & $3.1 \times 10^{0}$ & $2.0 \times 10^{0}$ & $8.4 \times 10^{-1}$ & $8.8 \times 10^{-1}$ \\
\hline $0-15$ & 8 & $4.6 \times 10^{-1}$ & $4.9 \times 10^{0}$ & $1.6 \times 10^{0}$ & $2.1 \times 10^{0}$ & $1.7 \times 10^{0}$ & $3.9 \times 10^{-1}$ & $9.0 \times 10^{-1}$ \\
\hline $0-25$ & 6 & $3.2 \times 10^{-1}$ & $1.5 \times 10^{0}$ & $7.6 \times 10^{-1}$ & $7.8 \times 10^{-1}$ & $4.5 \times 10^{-1}$ & $-4.1 \times 10^{-1}$ & $6.3 \times 10^{-1}$ \\
\hline $0-40$ & 5 & $2.1 \times 10^{-1}$ & $9.2 \times 10^{-1}$ & $3.7 \times 10^{-1}$ & $4.8 \times 10^{-1}$ & $3.1 \times 10^{-1}$ & $-9.2 \times 10^{-1}$ & $6.7 \times 10^{-1}$ \\
\hline $0-60$ & 5 & $1.4 \times 10^{-1}$ & $6.4 \times 10^{-1}$ & $2.8 \times 10^{-1}$ & $3.5 \times 10^{-1}$ & $2.3 \times 10^{-1}$ & $-1.2 \times 10^{0}$ & $7.0 \times 10^{-1}$ \\
\hline
\end{tabular}

NOTE: Specific activity is decay corrected to 1994 .

$\mathrm{N}^{\mathrm{a}}$ stands for number of individual samples. 
Table C-17. Americium-241 radionuclide concentration summary for all soil profiles taken from our most recent trip in 1991 on Busch Island (F38).

\begin{tabular}{|c|c|c|c|c|c|c|c|c|}
\hline \multirow{2}{*}{$\begin{array}{r}\text { Soil } \\
\text { depth } \\
(\mathrm{cm})\end{array}$} & \multirow[b]{2}{*}{$\mathrm{Na}^{\mathrm{a}}$} & \multicolumn{5}{|c|}{ pCig $^{-1}$ dry wt } & \multirow{2}{*}{$\begin{array}{l}\text { Mean } \\
\text { of logs }\end{array}$} & \multirow{2}{*}{$\begin{array}{r}\mathrm{SD} \\
\text { of logs } \\
\end{array}$} \\
\hline & & Minimum & Maximum & Median & Mean & SD & & \\
\hline $0-05$ & 4 & $1.0 \times 10^{0}$ & $1.6 \times 10^{1}$ & $5.4 \times 10^{0}$ & $6.9 \times 10^{0}$ & $7.2 \times 10^{0}$ & $1.3 \times 10^{0}$ & $1.4 \times 10^{0}$ \\
\hline $05-10$ & 2 & $4.1 \times 10^{0}$ & $7.9 \times 10^{0}$ & $6.0 \times 10^{0}$ & $6.0 \times 10^{0}$ & $2.7 \times 10^{0}$ & $1.7 \times 10^{0}$ & $4.7 \times 10^{-1}$ \\
\hline $10-15$ & 3 & $2.1 \times 10^{0}$ & $4.5 \times 10^{0}$ & $2.6 \times 10^{0}$ & $3.1 \times 10^{0}$ & $1.2 \times 10^{0}$ & $1.1 \times 10^{0}$ & $3.8 \times 10^{-1}$ \\
\hline $15-25$ & 3 & $5.0 \times 10^{-1}$ & $1.1 \times 10^{0}$ & $5.1 \times 10^{-1}$ & $6.9 \times 10^{-1}$ & $3.1 \times 10^{-1}$ & $-4.4 \times 10^{-1}$ & $4.2 \times 10^{-1}$ \\
\hline $25-40$ & 1 & $4.3 \times 10^{-1}$ & $4.3 \times 10^{-1}$ & $4.3 \times 10^{-1}$ & $4.3 \times 10^{-1}$ & $0.0 \times 10^{0}$ & $-8.6 \times 10^{-1}$ & $0.0 \times 10^{0}$ \\
\hline $40-60$ & 0 & $0.0 \times 10^{0}$ & $0.0 \times 10^{0}$ & $0.0 \times 10^{0}$ & $0.0 \times 10^{0}$ & $0.0 \times 10^{0}$ & $0.0 \times 10^{0}$ & $0.0 \times 10^{0}$ \\
\hline $0-05$ & 4 & $1.0 \times 10^{0}$ & $1.6 \times 10^{1}$ & $5.4 \times 10^{0}$ & $6.9 \times 10^{0}$ & $7.2 \times 10^{0}$ & $1.3 \times 10^{0}$ & $1.4 \times 10^{0}$ \\
\hline $0-10$ & 2 & $2.6 \times 10^{0}$ & $4.5 \times 10^{0}$ & $3.5 \times 10^{0}$ & $3.5 \times 10^{0}$ & $1.3 \times 10^{0}$ & $1.2 \times 10^{0}$ & $3.8 \times 10^{-1}$ \\
\hline $0-15$ & 2 & $2.5 \times 10^{0}$ & $4.5 \times 10^{0}$ & $3.5 \times 10^{0}$ & $3.5 \times 10^{0}$ & $1.4 \times 10^{0}$ & $1.2 \times 10^{0}$ & $4.3 \times 10^{-1}$ \\
\hline $0-25$ & 2 & $1.9 \times 10^{0}$ & $2.9 \times 10^{0}$ & $2.4 \times 10^{0}$ & $2.4 \times 10^{0}$ & $7.0 \times 10^{-1}$ & $8.5 \times 10^{-1}$ & $3.0 \times 10^{-1}$ \\
\hline $0-40$ & 1 & $1.3 \times 10^{0}$ & $1.3 \times 10^{0}$ & $1.3 \times 10^{0}$ & $1.3 \times 10^{0}$ & $0.0 \times 10^{0}$ & $2.9 \times 10^{-1}$ & $0.0 \times 10^{0}$ \\
\hline $0-60$ & 0 & $0.0 \times 10^{\circ}$ & $0.0 \times 10^{0}$ & $0.0 \times 10^{0}$ & $0.0 \times 10^{0}$ & $0.0 \times 10^{0}$ & $0.0 \times 10^{0}$ & $0.0 \times 10^{0}$ \\
\hline
\end{tabular}

NOTE: Specific activity is decay corrected to 1994.

$\mathrm{N}^{\mathrm{a}}$ stands for number of individual samples. 
Table C-18. Americium-241 radionuclide concentration summary for all soil profiles taken from the 1978 NMIRS together with our most recent trips in 1986 through 1989 on Rongelap Island (F42).

\begin{tabular}{|c|c|c|c|c|c|c|c|c|}
\hline \multirow{2}{*}{$\begin{array}{l}\text { Soil } \\
\text { depth } \\
(\mathrm{cm})\end{array}$} & \multirow[b]{2}{*}{$\mathrm{Na}$} & \multicolumn{5}{|c|}{ pCi $g^{-1}$ dry wt } & \multirow{2}{*}{$\begin{array}{l}\text { Mean } \\
\text { of logs }\end{array}$} & \multirow{2}{*}{$\begin{array}{c}S D \\
\text { of logs } \\
\end{array}$} \\
\hline & & Minimum & Maximum & Median & Mean & SD & & \\
\hline $0-05$ & 267 & $4.0 \times 10^{-2}$ & $1.1 \times 10^{1}$ & $1.6 \times 10^{0}$ & $2.0 \times 10^{0}$ & $1.7 \times 10^{0}$ & $3.0 \times 10^{-1}$ & $9.7 \times 10^{-1}$ \\
\hline $05-10$ & 84 & $4.9 \times 10^{-2}$ & $7.2 \times 10^{0}$ & $6.8 \times 10^{-1}$ & $1.3 \times 10^{0}$ & $1.5 \times 10^{0}$ & $-2.8 \times 10^{-1}$ & $1.0 \times 10^{0}$ \\
\hline $10-15$ & 51 & $7.6 \times 10^{-4}$ & $2.6 \times 10^{0}$ & $4.8 \times 10^{-1}$ & $6.1 \times 10^{-1}$ & $5.5 \times 10^{-1}$ & $-1.1 \times 10^{0}$ & $1.4 \times 10^{0}$ \\
\hline $15-25$ & 35 & $7.5 \times 10^{-3}$ & $1.4 \times 10^{0}$ & $1.8 \times 10^{-1}$ & $3.1 \times 10^{-1}$ & $3.6 \times 10^{-1}$ & $-1.8 \times 10^{0}$ & $1.2 \times 10^{0}$ \\
\hline $25-40$ & 22 & $7.5 \times 10^{-4}$ & $4.4 \times 10^{-1}$ & $4.6 \times 10^{-2}$ & $7.9 \times 10^{-2}$ & $9.9 \times 10^{-2}$ & $-3.4 \times 10^{0}$ & $1.6 \times 10^{0}$ \\
\hline $40-60$ & 17 & $1.2 \times 10^{-3}$ & $4.0 \times 10^{-1}$ & $2.0 \times 10^{-2}$ & $7.1 \times 10^{-2}$ & $1.1 \times 10^{-1}$ & $-4.0 \times 10^{0}$ & $1.9 \times 10^{0}$ \\
\hline $0-05$ & 267 & $4.0 \times 10^{-2}$ & $1.1 \times 10^{1}$ & $1.6 \times 10^{0}$ & $2.0 \times 10^{0}$ & $1.7 \times 10^{0}$ & $3.0 \times 10^{-1}$ & $9.7 \times 10^{-1}$ \\
\hline $0-10$ & 78 & $4.4 \times 10^{-2}$ & $8.0 \times 10^{0}$ & $1.7 \times 10^{0}$ & $2.2 \times 10^{0}$ & $1.6 \times 10^{0}$ & $4.5 \times 10^{-1}$ & $9.2 \times 10^{-1}$ \\
\hline $0-15$ & 41 & $1.3 \times 10^{-1}$ & $5.6 \times 10^{0}$ & $1.4 \times 10^{0}$ & $1.6 \times 10^{0}$ & $1.3 \times 10^{0}$ & $1.8 \times 10^{-1}$ & $8.6 \times 10^{-1}$ \\
\hline $0-25$ & 21 & $9.4 \times 10^{-2}$ & $3.5 \times 10^{0}$ & $8.7 \times 10^{-1}$ & $1.0 \times 10^{0}$ & $9.4 \times 10^{-1}$ & $-4.0 \times 10^{-1}$ & $9.9 \times 10^{-1}$ \\
\hline $0-40$ & 10 & $6.5 \times 10^{-2}$ & $9.8 \times 10^{-1}$ & $3.2 \times 10^{-1}$ & $3.8 \times 10^{-1}$ & $2.8 \times 10^{-1}$ & $-1.3 \times 10^{0}$ & $8.9 \times 10^{-1}$ \\
\hline $0-60$ & 7 & $4.4 \times 10^{-2}$ & $7.3 \times 10^{-1}$ & $1.8 \times 10^{-1}$ & $2.5 \times 10^{-1}$ & $2.4 \times 10^{-1}$ & $-1.8 \times 10^{0}$ & $1.0 \times 10^{0}$ \\
\hline
\end{tabular}

NOTE: Specific activity is decay corrected to 1994 .

$\mathrm{N}^{\mathrm{a}}$ stands for number of individual samples. 
Table C-19. Americium-241 radionuclide concentration summary for all soil profiles taken from the 1978 NMIRS together with our most recent trip in 1991 on Arbar Island (F43).

\begin{tabular}{|c|c|c|c|c|c|c|c|c|}
\hline \multirow{2}{*}{$\begin{array}{r}\text { Soil } \\
\text { depth } \\
(\mathrm{cm})\end{array}$} & \multirow[b]{2}{*}{$\mathrm{Na}$} & \multicolumn{5}{|c|}{ pCi $g^{-1}$ dry wt } & \multirow{2}{*}{$\begin{array}{l}\text { Mean } \\
\text { of logs }\end{array}$} & \multirow{2}{*}{$\begin{array}{c}S D \\
\text { of } \operatorname{logs} \\
\end{array}$} \\
\hline & & Minimum & Maximum & Median & Mean & $\mathrm{SD}$ & & \\
\hline $0-05$ & 8 & $2.3 \times 10^{0}$ & $1.6 \times 10^{1}$ & $3.5 \times 10^{0}$ & $7.0 \times 10^{0}$ & $5.7 \times 10^{0}$ & $1.7 \times 10^{0}$ & $8.2 \times 10^{-1}$ \\
\hline $05-10$ & 6 & $3.5 \times 10^{-1}$ & $7.1 \times 10^{0}$ & $9.5 \times 10^{-1}$ & $2.2 \times 10^{0}$ & $2.7 \times 10^{0}$ & $1.8 \times 10^{-1}$ & $1.2 \times 10^{0}$ \\
\hline $10-15$ & 2 & $5.9 \times 10^{-1}$ & $4.7 \times 10^{0}$ & $2.7 \times 10^{0}$ & $2.7 \times 10^{0}$ & $2.9 \times 10^{0}$ & $5.1 \times 10^{-1}$ & $1.5 \times 10^{0}$ \\
\hline $15-25$ & 1 & $2.9 \times 10^{0}$ & $2.9 \times 10^{0}$ & $2.9 \times 10^{0}$ & $2.9 \times 10^{0}$ & $0.0 \times 10^{0}$ & $1.1 \times 10^{0}$ & $0.0 \times 10^{0}$ \\
\hline $25-40$ & 0 & $0.0 \times 10^{0}$ & $0.0 \times 10^{0}$ & $0.0 \times 10^{0}$ & $0.0 \times 10^{0}$ & $0.0 \times 10^{0}$ & $0.0 \times 10^{0}$ & $0.0 \times 10^{0}$ \\
\hline $40-60$ & 0 & $0.0 \times 10^{0}$ & $0.0 \times 10^{0}$ & $0.0 \times 10^{0}$ & $0.0 \times 10^{0}$ & $0.0 \times 10^{0}$ & $0.0 \times 10^{0}$ & $0.0 \times 10^{0}$ \\
\hline $0-05$ & 8 & $2.3 \times 10^{0}$ & $1.6 \times 10^{1}$ & $3.5 \times 10^{0}$ & $7.0 \times 10^{0}$ & $5.7 \times 10^{0}$ & $1.7 \times 10^{0}$ & $8.2 \times 10^{-1}$ \\
\hline $0-10$ & 6 & $1.5 \times 10^{0}$ & $9.8 \times 10^{0}$ & $2.7 \times 10^{0}$ & $4.1 \times 10^{0}$ & $3.4 \times 10^{0}$ & $1.2 \times 10^{0}$ & $7.8 \times 10^{-1}$ \\
\hline $0-15$ & 2 & $1.4 \times 10^{0}$ & $3.9 \times 10^{0}$ & $2.7 \times 10^{0}$ & $2.7 \times 10^{0}$ & $1.7 \times 10^{0}$ & $8.6 \times 10^{-1}$ & $7.0 \times 10^{-1}$ \\
\hline $0-25$ & 1 & $3.5 \times 10^{0}$ & $3.5 \times 10^{0}$ & $3.5 \times 10^{0}$ & $3.5 \times 10^{0}$ & $0.0 \times 10^{0}$ & $1.3 \times 10^{0}$ & $0.0 \times 10^{0}$ \\
\hline $0-40$ & 0 & $0.0 \times 10^{0}$ & $0.0 \times 10^{0}$ & $0.0 \times 10^{0}$ & $0.0 \times 10^{0}$ & $0.0 \times 10^{0}$ & $0.0 \times 10^{0}$ & $0.0 \times 10^{0}$ \\
\hline $0-60$ & 0 & $0.0 \times 10^{0}$ & $0.0 \times 10^{0}$ & $0.0 \times 10^{0}$ & $0.0 \times 10^{0}$ & $0.0 \times 10^{0}$ & $0.0 \times 10^{0}$ & $0.0 \times 10^{0}$ \\
\hline
\end{tabular}

NOTE: Specific activity is decay corrected to 1994.

$\mathrm{Na}^{\mathrm{a}}$ stands for number of individual samples. 
Table C-20. Americium-241 radionuclide concentration summary for all soil profiles taken from our most recent trip in 1991 on Eniran Island (F46).

\begin{tabular}{|c|c|c|c|c|c|c|c|c|}
\hline \multirow{2}{*}{$\begin{array}{l}\text { Soil } \\
\text { depth } \\
(\mathrm{cm})\end{array}$} & \multirow[b]{2}{*}{$\mathrm{Na}$} & \multicolumn{5}{|c|}{ pCi $g^{-1}$ dry wt } & \multirow{2}{*}{$\begin{array}{l}\text { Mean } \\
\text { of logs }\end{array}$} & \multirow{2}{*}{$\begin{array}{c}\text { SD } \\
\text { of logs } \\
\end{array}$} \\
\hline & & Minimum & Maximum & Median & Mean & $\mathrm{SD}$ & & \\
\hline $0-05$ & 5 & $1.6 \times 10^{0}$ & $7.7 \times 10^{0}$ & $5.0 \times 10^{0}$ & $4.8 \times 10^{0}$ & $2.2 \times 10^{0}$ & $1.4 \times 10^{0}$ & $5.9 \times 10^{-1}$ \\
\hline $05-10$ & 2 & $1.2 \times 10^{0}$ & $2.4 \times 10^{0}$ & $1.8 \times 10^{0}$ & $1.8 \times 10^{0}$ & $9.0 \times 10^{-1}$ & $5.2 \times 10^{-1}$ & $5.2 \times 10^{-1}$ \\
\hline $10-15$ & 2 & $1.9 \times 10^{-1}$ & $1.0 \times 10^{0}$ & $6.0 \times 10^{-1}$ & $6.0 \times 10^{-1}$ & $5.8 \times 10^{-1}$ & $-8.4 \times 10^{-1}$ & $1.2 \times 10^{0}$ \\
\hline $15-25$ & 0 & $0.0 \times 10^{0}$ & $0.0 \times 10^{0}$ & $0.0 \times 10^{0}$ & $0.0 \times 10^{0}$ & $0.0 \times 10^{0}$ & $0.0 \times 10^{0}$ & $0.0 \times 10^{0}$ \\
\hline $25-40$ & 0 & $0.0 \times 10^{0}$ & $0.0 \times 10^{0}$ & $0.0 \times 10^{0}$ & $0.0 \times 10^{0}$ & $0.0 \times 10^{0}$ & $0.0 \times 10^{0}$ & $0.0 \times 10^{0}$ \\
\hline $40-60$ & 0 & $0.0 \times 10^{0}$ & $0.0 \times 10^{0}$ & $0.0 \times 10^{0}$ & $0.0 \times 10^{0}$ & $0.0 \times 10^{0}$ & $0.0 \times 10^{0}$ & $0.0 \times 10^{0}$ \\
\hline $0-05$ & 5 & $1.6 \times 10^{0}$ & $7.7 \times 10^{0}$ & $5.0 \times 10^{0}$ & $4.8 \times 10^{0}$ & $2.2 \times 10^{0}$ & $1.4 \times 10^{0}$ & $5.9 \times 10^{-1}$ \\
\hline $0-10$ & 2 & $2.0 \times 10^{0}$ & $2.5 \times 10^{0}$ & $2.3 \times 10^{0}$ & $2.3 \times 10^{0}$ & $3.4 \times 10^{-1}$ & $8.2 \times 10^{-1}$ & $1.5 \times 10^{-1}$ \\
\hline $0-15$ & 2 & $1.7 \times 10^{0}$ & $1.7 \times 10^{0}$ & $1.7 \times 10^{0}$ & $1.7 \times 10^{0}$ & $3.2 \times 10^{-2}$ & $5.4 \times 10^{-1}$ & $1.9 \times 10^{-2}$ \\
\hline $0-25$ & 0 & $0.0 \times 10^{0}$ & $0.0 \times 10^{0}$ & $0.0 \times 10^{0}$ & $0.0 \times 10^{0}$ & $0.0 \times 10^{0}$ & $0.0 \times 10^{0}$ & $0.0 \times 10^{0}$ \\
\hline $0-40$ & 0 & $0.0 \times 10^{0}$ & $0.0 \times 10^{0}$ & $0.0 \times 10^{0}$ & $0.0 \times 10^{0}$ & $0.0 \times 10^{0}$ & $0.0 \times 10^{0}$ & $0.0 \times 10^{0}$ \\
\hline $0-60$ & 0 & $0.0 \times 10^{0}$ & $0.0 \times 10^{0}$ & $0.0 \times 10^{0}$ & $0.0 \times 10^{0}$ & $0.0 \times 10^{0}$ & $0.0 \times 10^{0}$ & $0.0 \times 10^{0}$ \\
\hline
\end{tabular}

NOTE: Specific activity is decay corrected to 1994.

$\mathrm{Na}$ stands for number of individual samples. 
Table C-21. Americium-241 radionuclide concentration summary for all soil profiles taken from our most recent trip in 1991 on Tufa Island (F47).

\begin{tabular}{|c|c|c|c|c|c|c|c|c|}
\hline \multirow{2}{*}{$\begin{array}{r}\text { Soil } \\
\text { depth } \\
(\mathrm{cm})\end{array}$} & \multirow[b]{2}{*}{$\mathrm{Na}$} & \multicolumn{5}{|c|}{ pCi g-1 dry wt } & \multirow{2}{*}{$\begin{array}{l}\text { Mean } \\
\text { of logs } \\
\end{array}$} & \multirow{2}{*}{$\begin{array}{c}\mathrm{SD} \\
\text { of logs } \\
\end{array}$} \\
\hline & & Minimum & Maximum & Median & Mean & $\mathrm{SD}$ & & \\
\hline $0-05$ & 8 & $1.8 \times 10^{0}$ & $9.4 \times 10^{0}$ & $3.6 \times 10^{0}$ & $4.5 \times 10^{0}$ & $2.6 \times 10^{0}$ & $1.4 \times 10^{0}$ & $5.6 \times 10^{-1}$ \\
\hline $05-10$ & 6 & $7.0 \times 10^{-1}$ & $1.6 \times 10^{1}$ & $2.1 \times 10^{0}$ & $5.2 \times 10^{0}$ & $6.0 \times 10^{0}$ & $1.1 \times 10^{0}$ & $1.2 \times 10^{0}$ \\
\hline $10-15$ & 4 & $1.6 \times 10^{-1}$ & $2.3 \times 10^{0}$ & $1.4 \times 10^{0}$ & $1.3 \times 10^{0}$ & $8.8 \times 10^{-1}$ & $-1.1 \times 10^{-1}$ & $1.2 \times 10^{0}$ \\
\hline $15-25$ & 4 & $1.7 \times 10^{-1}$ & $9.4 \times 10^{-1}$ & $2.0 \times 10^{-1}$ & $3.8 \times 10^{-1}$ & $3.8 \times 10^{-1}$ & $-1.3 \times 10^{0}$ & $8.2 \times 10^{-1}$ \\
\hline $25-40$ & 0 & $0.0 \times 10^{0}$ & $0.0 \times 10^{0}$ & $0.0 \times 10^{0}$ & $0.0 \times 10^{0}$ & $0.0 \times 10^{0}$ & $0.0 \times 10^{0}$ & $0.0 \times 10^{0}$ \\
\hline $40-60$ & 0 & $0.0 \times 10^{0}$ & $0.0 \times 10^{0}$ & $0.0 \times 10^{0}$ & $0.0 \times 10^{0}$ & $0.0 \times 10^{0}$ & $0.0 \times 10^{0}$ & $0.0 \times 10^{0}$ \\
\hline $0-05$ & 8 & $1.8 \times 10^{0}$ & $9.4 \times 10^{0}$ & $3.6 \times 10^{0}$ & $4.5 \times 10^{0}$ & $2.6 \times 10^{0}$ & $1.4 \times 10^{0}$ & $5.6 \times 10^{-1}$ \\
\hline $0-10$ & 6 & $1.9 \times 10^{0}$ & $9.6 \times 10^{0}$ & $3.5 \times 10^{0}$ & $4.7 \times 10^{0}$ & $3.2 \times 10^{0}$ & $1.3 \times 10^{0}$ & $6.8 \times 10^{-1}$ \\
\hline $0-15$ & 4 & $1.3 \times 10^{0}$ & $7.2 \times 10^{0}$ & $3.6 \times 10^{0}$ & $3.9 \times 10^{0}$ & $2.8 \times 10^{0}$ & $1.1 \times 10^{0}$ & $8.2 \times 10^{-1}$ \\
\hline $0-25$ & 3 & $8.5 \times 10^{-1}$ & $4.7 \times 10^{0}$ & $3.3 \times 10^{0}$ & $2.9 \times 10^{0}$ & $1.9 \times 10^{0}$ & $8.5 \times 10^{-1}$ & $9.0 \times 10^{-1}$ \\
\hline $0-40$ & 0 & $0.0 \times 10^{0}$ & $0.0 \times 10^{0}$ & $0.0 \times 10^{0}$ & $0.0 \times 10^{0}$ & $0.0 \times 10^{0}$ & $0.0 \times 10^{0}$ & $0.0 \times 10^{0}$ \\
\hline $0-60$ & 0 & $0.0 \times 10^{0}$ & $0.0 \times 10^{0}$ & $0.0 \times 10^{0}$ & $0.0 \times 10^{0}$ & $0.0 \times 10^{0}$ & $0.0 \times 10^{0}$ & $0.0 \times 10^{0}$ \\
\hline
\end{tabular}

NOTE: Specific activity is decay corrected to 1994.

$\mathrm{Na}$ stands for number of individual samples. 
Table C-22. Americium-241 radionuclide concentration summary for all soil profiles taken from the 1978 NMIRS together with our most recent trip in 1989 on Borukka Island (F49).

\begin{tabular}{|c|c|c|c|c|c|c|c|c|}
\hline \multirow{2}{*}{$\begin{array}{r}\text { Soil } \\
\text { depth } \\
(\mathrm{cm})\end{array}$} & \multirow[b]{2}{*}{$\mathrm{Na}$} & \multicolumn{5}{|c|}{ pCi $g^{-1}$ dry wt } & \multirow{2}{*}{$\begin{array}{l}\text { Mean } \\
\text { of logs }\end{array}$} & \multirow{2}{*}{$\begin{array}{r}S D \\
\text { of logs } \\
\end{array}$} \\
\hline & & Minimum & Maximum & Median & Mean & SD & & \\
\hline $0-05$ & 13 & $1.1 \times 10^{0}$ & $1.1 \times 10^{1}$ & $3.2 \times 10^{0}$ & $4.5 \times 10^{0}$ & $2.9 \times 10^{0}$ & $1.3 \times 10^{0}$ & $6.2 \times 10^{-1}$ \\
\hline $05-10$ & 9 & $1.6 \times 10^{-1}$ & $1.5 \times 10^{1}$ & $8.8 \times 10^{-1}$ & $2.6 \times 10^{0}$ & $4.8 \times 10^{0}$ & $5.3 \times 10^{-2}$ & $1.3 \times 10^{0}$ \\
\hline $10-15$ & 8 & $9.9 \times 10^{-2}$ & $2.3 \times 10^{0}$ & $6.1 \times 10^{-1}$ & $7.6 \times 10^{-1}$ & $7.4 \times 10^{-1}$ & $-7.6 \times 10^{-1}$ & $1.1 \times 10^{0}$ \\
\hline $15-25$ & 9 & $3.9 \times 10^{-2}$ & $8.8 \times 10^{-1}$ & $2.0 \times 10^{-1}$ & $2.6 \times 10^{-1}$ & $2.5 \times 10^{-1}$ & $-1.7 \times 10^{0}$ & $9.0 \times 10^{-1}$ \\
\hline $25-40$ & 5 & $1.6 \times 10^{-2}$ & $2.9 \times 10^{-1}$ & $3.5 \times 10^{-2}$ & $9.2 \times 10^{-2}$ & $1.2 \times 10^{-1}$ & $-3.0 \times 10^{0}$ & $1.2 \times 10^{0}$ \\
\hline $40-60$ & 0 & $0.0 \times 10^{0}$ & $0.0 \times 10^{0}$ & $0.0 \times 10^{0}$ & $0.0 \times 10^{0}$ & $0.0 \times 10^{0}$ & $0.0 \times 10^{0}$ & $0.0 \times 10^{0}$ \\
\hline $0-05$ & 13 & $1.1 \times 10^{0}$ & $1.1 \times 10^{1}$ & $3.2 \times 10^{0}$ & $4.5 \times 10^{0}$ & $2.9 \times 10^{0}$ & $1.3 \times 10^{0}$ & $6.2 \times 10^{-1}$ \\
\hline $0-10$ & 9 & $1.3 \times 10^{0}$ & $8.1 \times 10^{0}$ & $2.2 \times 10^{0}$ & $3.3 \times 10^{0}$ & $2.2 \times 10^{0}$ & $1.0 \times 10^{0}$ & $6.0 \times 10^{-1}$ \\
\hline $0-15$ & 6 & $8.9 \times 10^{-1}$ & $3.4 \times 10^{0}$ & $1.7 \times 10^{0}$ & $2.0 \times 10^{0}$ & $1.0 \times 10^{0}$ & $5.6 \times 10^{-1}$ & $5.4 \times 10^{-1}$ \\
\hline $0-25$ & 5 & $5.9 \times 10^{-1}$ & $2.2 \times 10^{0}$ & $8.2 \times 10^{-1}$ & $1.3 \times 10^{0}$ & $7.4 \times 10^{-1}$ & $8.2 \times 10^{-2}$ & $6.0 \times 10^{-1}$ \\
\hline $0-40$ & 3 & $3.7 \times 10^{-1}$ & $5.2 \times 10^{-1}$ & $4.8 \times 10^{-1}$ & $4.6 \times 10^{-1}$ & $7.7 \times 10^{-2}$ & $-7.9 \times 10^{-1}$ & $1.8 \times 10^{-1}$ \\
\hline $0-60$ & 0 & $0.0 \times 10^{0}$ & $0.0 \times 10^{0}$ & $0.0 \times 10^{0}$ & $0.0 \times 10^{0}$ & $0.0 \times 10^{0}$ & $0.0 \times 10^{0}$ & $0.0 \times 10^{0}$ \\
\hline
\end{tabular}

NOTE: Specific activity is decay corrected to 1994. $\mathrm{N}^{\mathrm{a}}$ stands for number of individual samples. 
Appendix D

Maps of Islands 


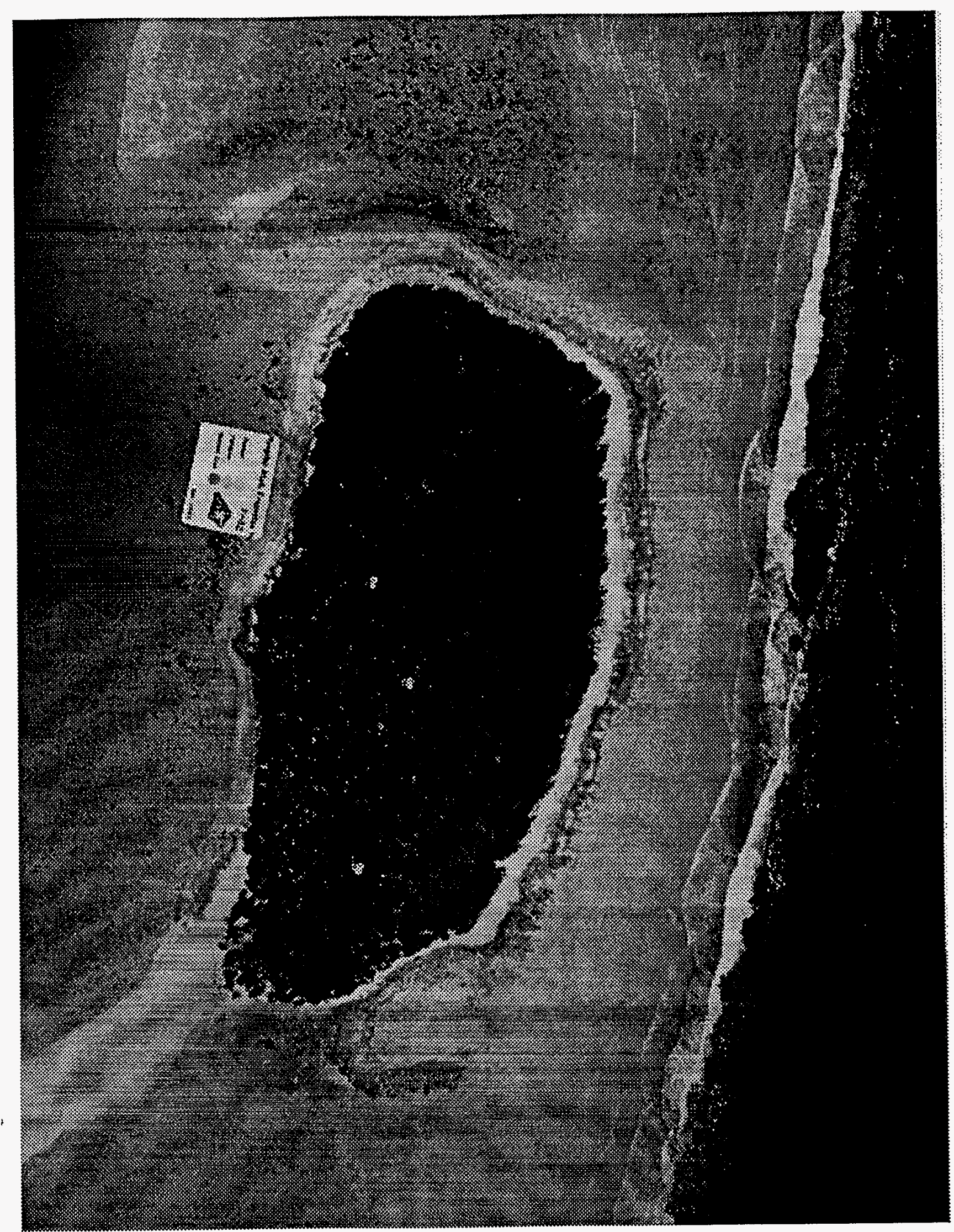




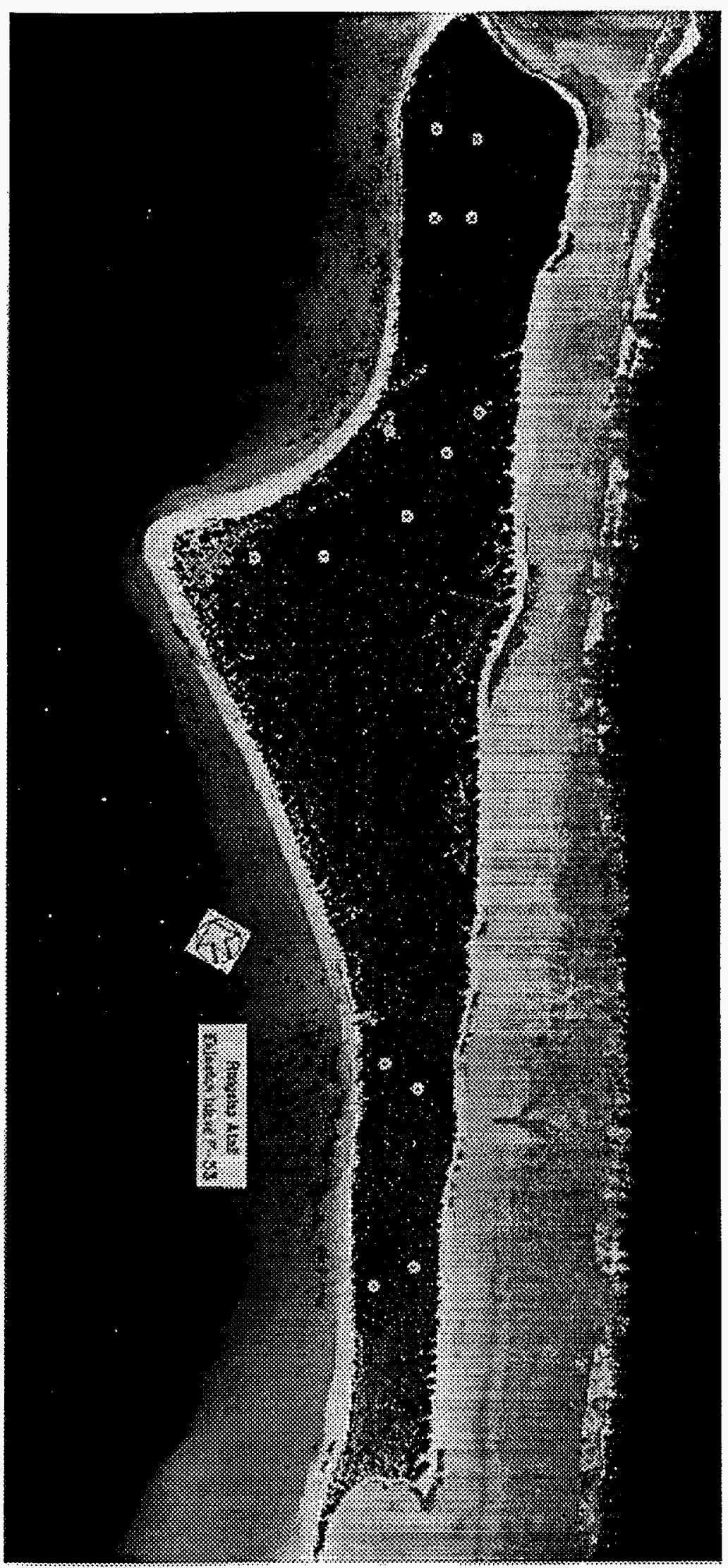




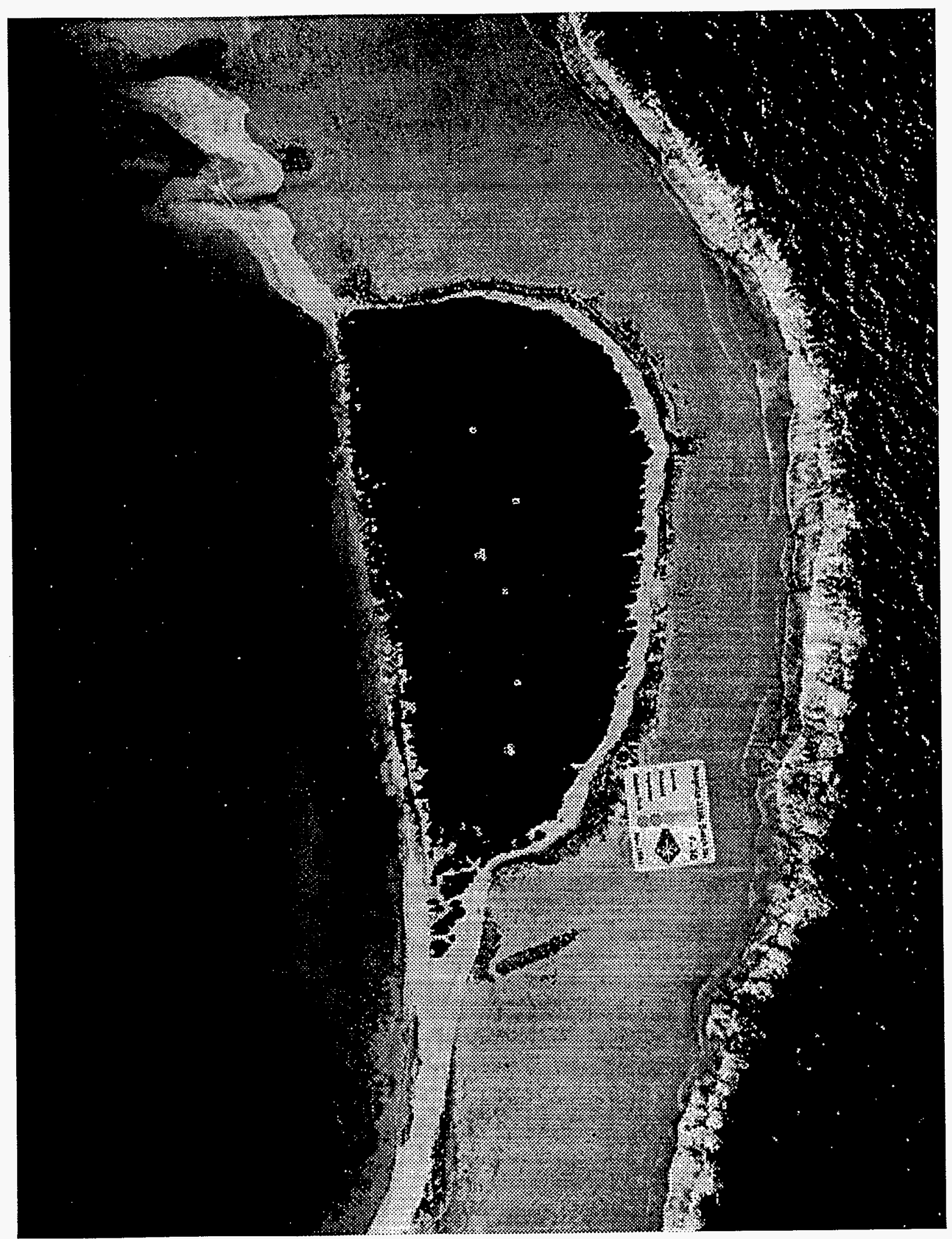




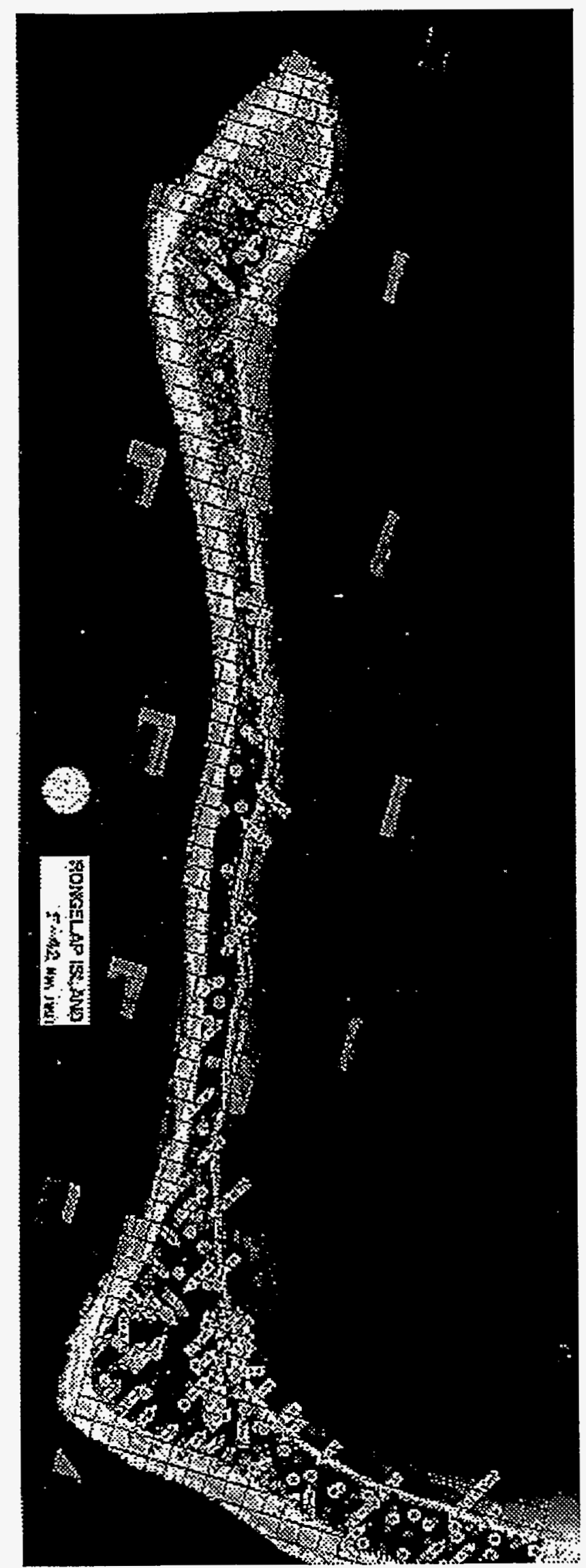




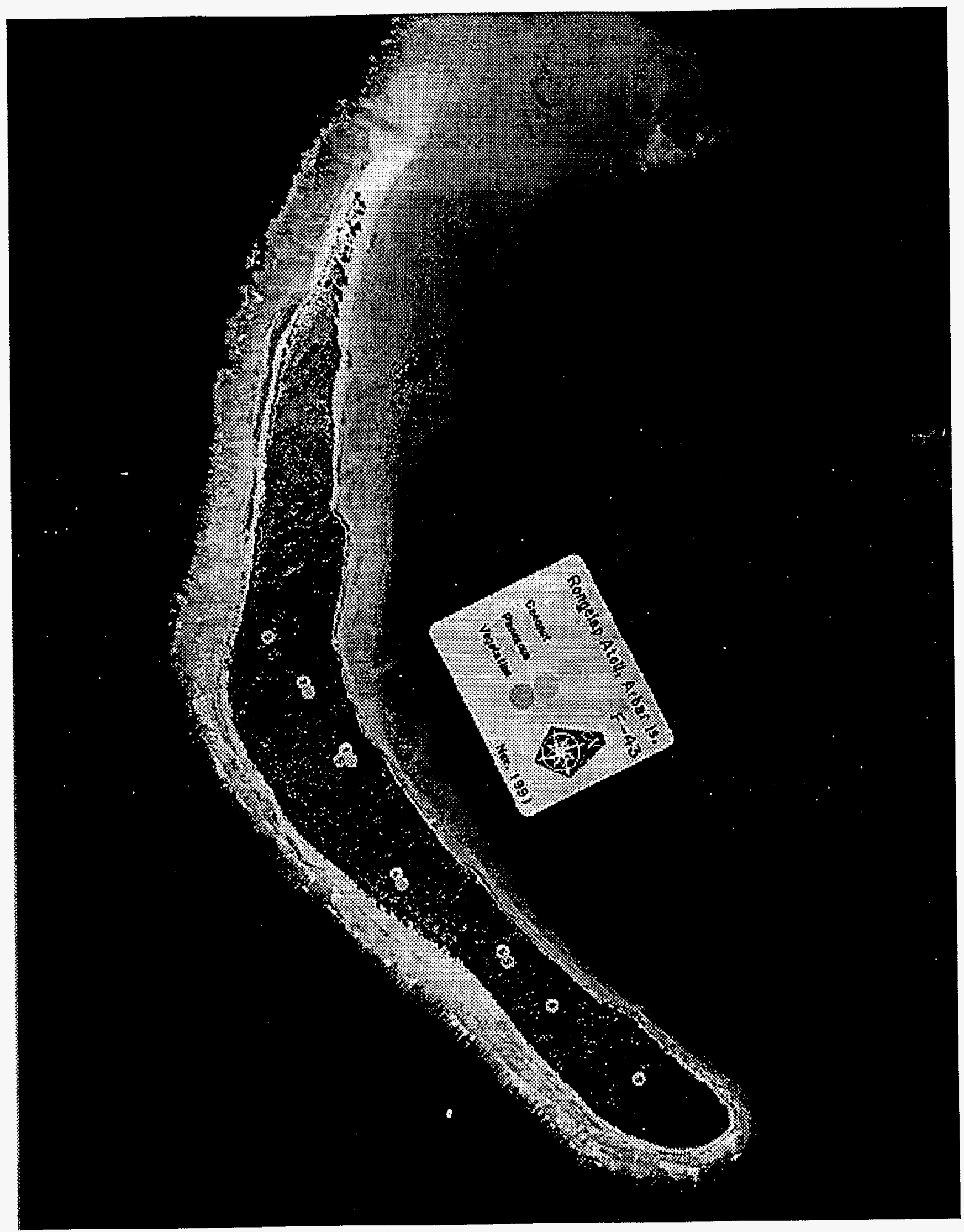




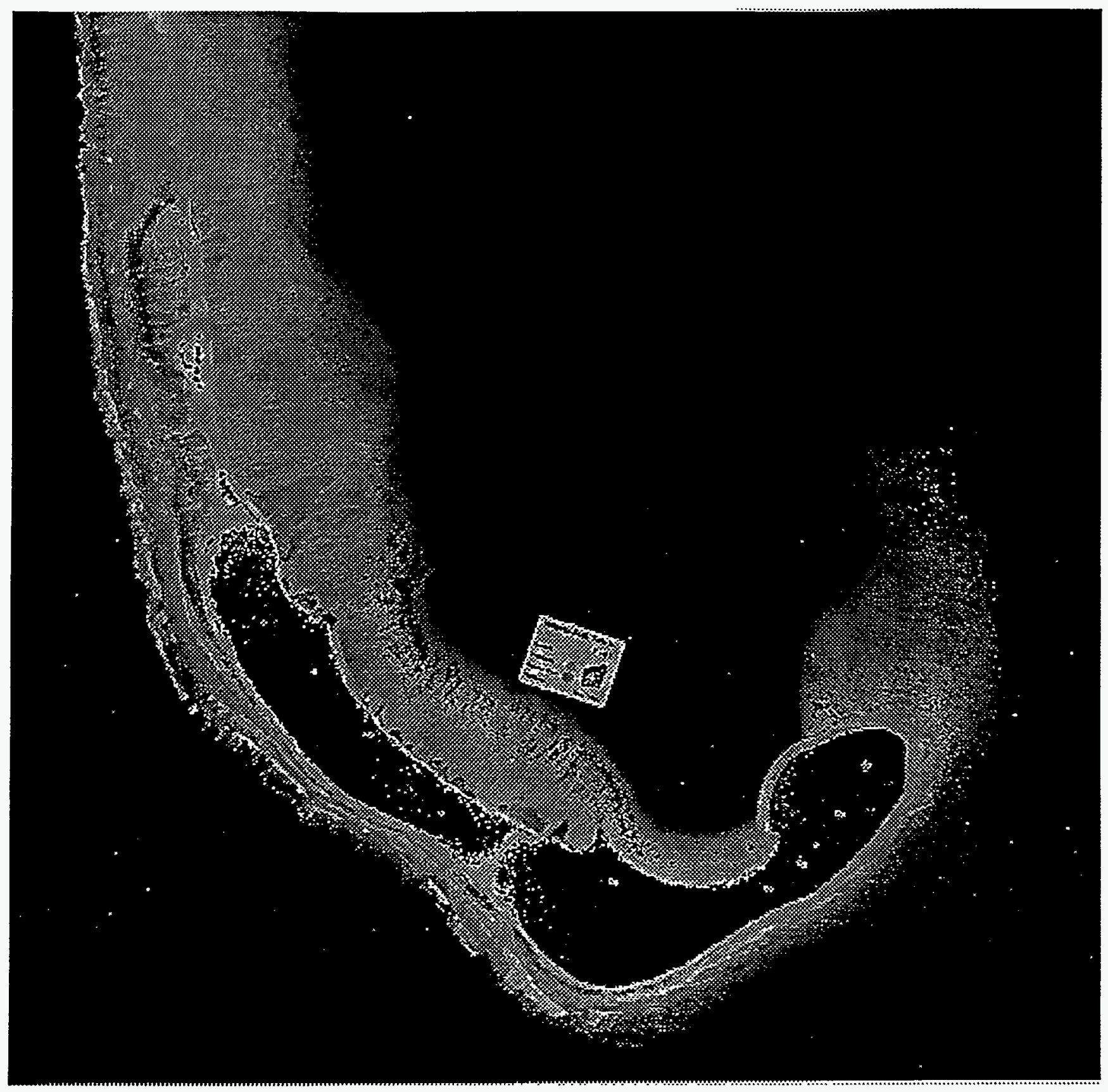




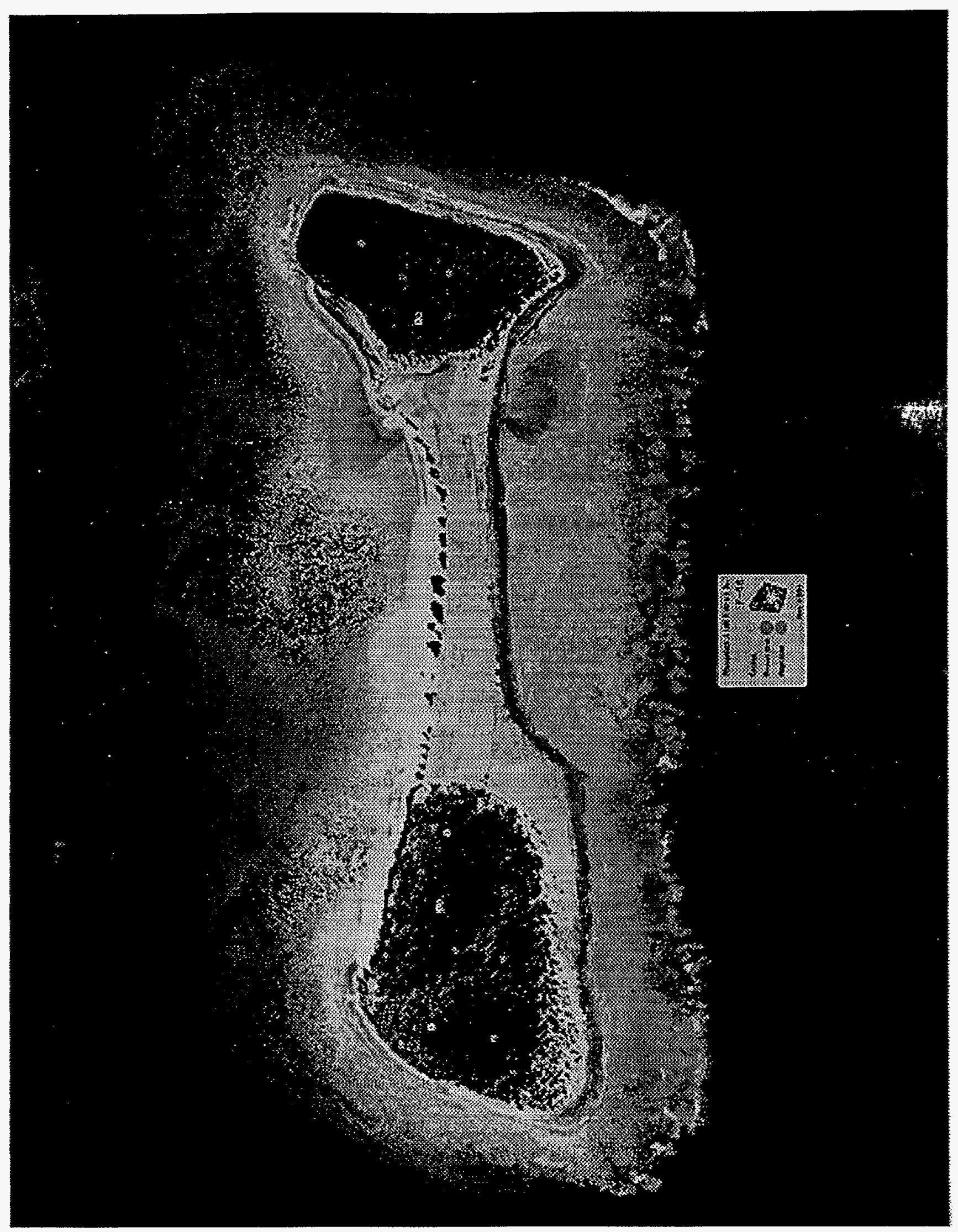




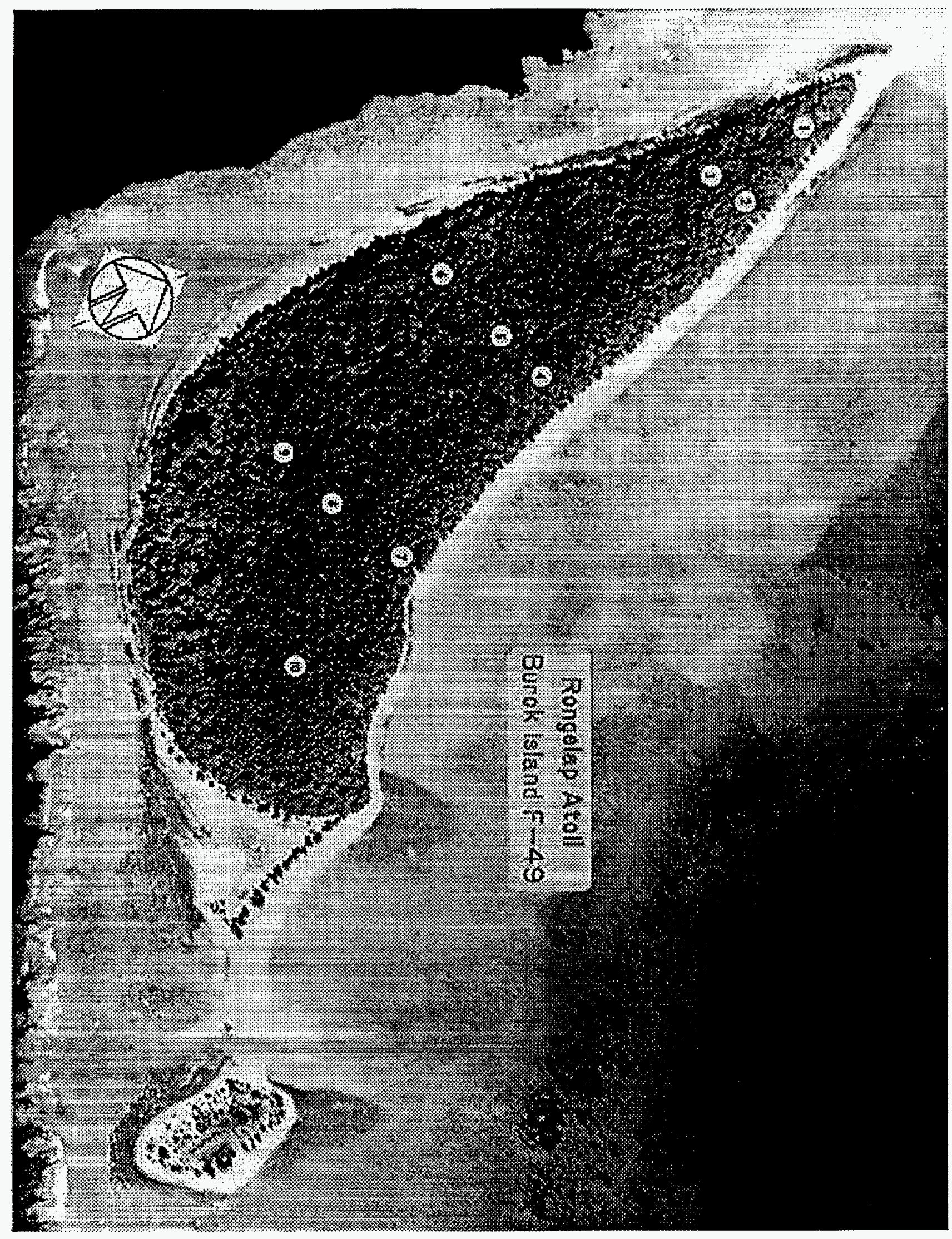

